Linköping Studies in Science and Technology

Dissertation No. 2194

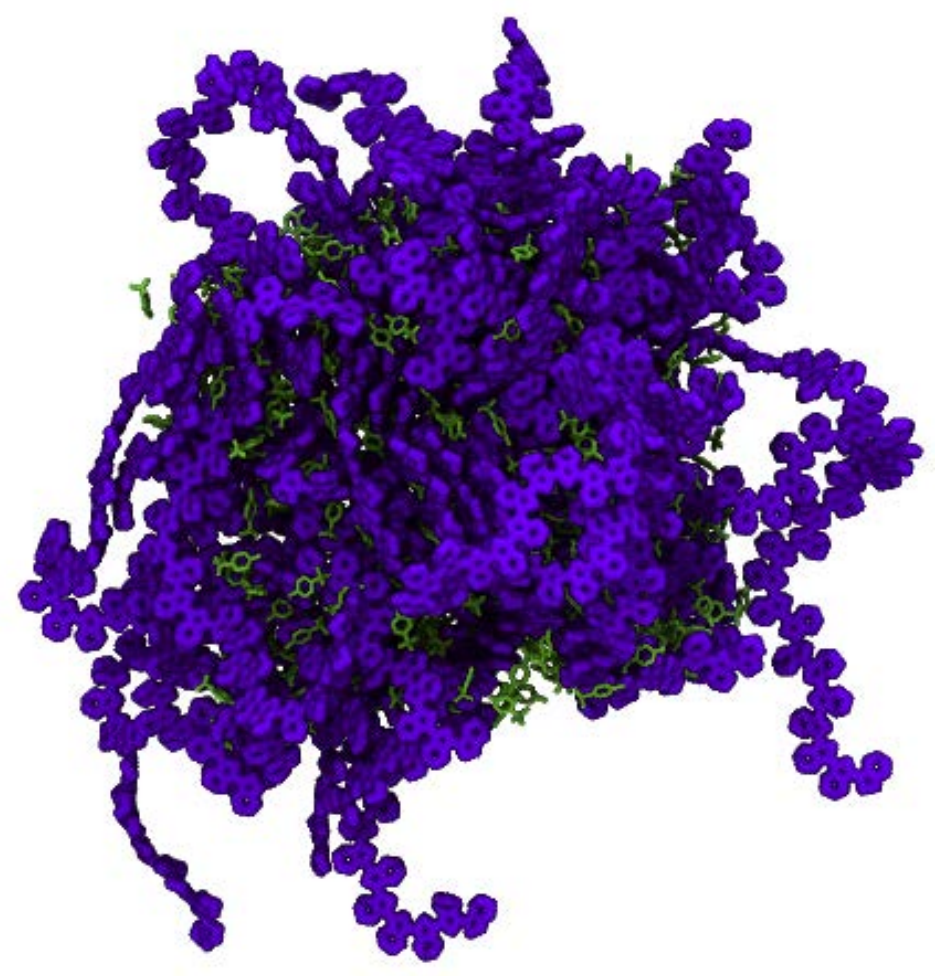

\title{
Computational study of Polymerization, Crystallization and Mechanical Properties of Conducting Polymers
}

\section{Donghyun Kim}

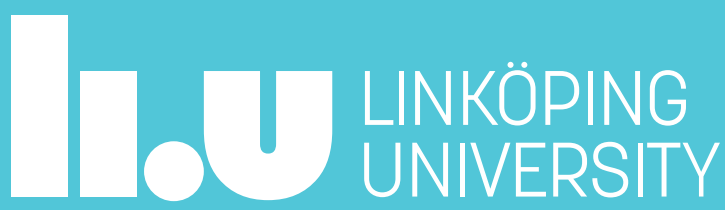



Doctor of Philosophy Doctoral thesis in Applied Physics

Dissertation No. 2194

Norrköping, 2021

Laboratory of Organic Electronics - Department of Science and Technology (ITN)

\section{Computational study of Polymerization, Crystallization and Mechanical Properties of Conducting Polymers}

Donghyun Kim

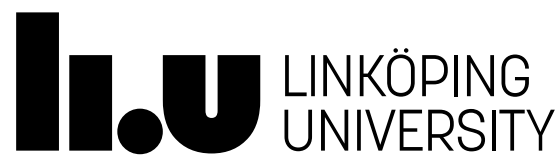


Description of the cover page: Depicts the nanostructure of PEDOT polymerized and dried at $373 \mathrm{~K}$ included in the thesis by Donghyun Kim, LiU.

\begin{tabular}{|l|l}
\hline$(\boldsymbol{c})$ EY \\
\hline
\end{tabular} International License.

https://creativecommons.org/licenses/by/4.0/

Linköping Studies in Science and Technology. Dissertation No. 2194

Copyright (C) 2021 Donghyun Kim, unless otherwise noted

Printed in Sweden by LiU-Tryck, Linköping, 2021

Electronic publication: www.ep.liu.se

ISSN: 0345-7524

ISBN: 978-91-7929-143-3 (tryckt) ISBN: 978-91-7929-144-0 (PDF)

Laboratory of Organic Electronics

Department of Science and Technology

Linköping University

Campus Norrköping

60174 Norrköping, Sweden 


\section{Abstract}

Nowadays, electronic devices that include conducting polymers, ranging from batteries and OLED panel for TVs and smartphones to bioelectronics devices such as sensors and ion-pumps for drug-delivery are widely used in our life. The use of conducting polymer in many electronic applications was driven by demand for light weight, flexibility or biocompatibility with the performance on pair with conventional inorganic counterparts. As a result, during last two decades conducting polymers have been a subject of significant interest in both academia and industry.

Though many aspects of conducting polymers' nature have been disclosed, it is still challenging to design a conducting polymer that meets required electrical and mechanical properties. It is because these properties are simultaneously influenced by many various factors such as charge carrier concentration, molecular weight, chemical structure. Thus, understanding the polymerization, crystallization and morphology of conducting polymers is a crucial key to realize flexible, stretchable or wearable electric applications based on conducting polymers. Computational methods represent an important tool in studies of conducting polymer since they not only provide information about morphology of polymer films on molecular level, but also can describe physical properties such as thermodynamic potential and pair-wise interaction between chains that experimental studies can rely on.

This thesis is focused on two classes of conducting polymers: Thiophene-based polymers (PEDOT and $\mathrm{p}\left(\mathrm{g}_{4} 2 \mathrm{~T}-\mathrm{T}\right)$ ) and NDI-based polymers (pNDI-TVT-TET). The former is one of the most versatile p-type materials, while the latter is known to have ambipolar charge transport owing to its donor-acceptor structure. First, we corroborated the mechanism of in-situ chemical polymerization of PEDOT with $\mathrm{Fe}(\mathrm{TOS})_{3}$ as oxidant by reaction energy calculation for the conventional oxidation polymerization mechanism. We found that doping of PEDOT chain became energetically unfavorable beyond of 33\% doping level and we explained it in terms of polaron localization. To explore the impact of polymerization temperature on PEDOT length, we developed a polymerization model for in-situ chemical polymerization of PEDOT:TOS. The results demonstrate that the average PEDOT length is 6, 7, and 11 monomer units at 298, 323 , and $373 \mathrm{~K}$ respectively, and we concluded that the diffusivity of reactants was a dominant factor determining the PEDOT length.

We also investigated the effect of molecular doping on the morphology of $\mathrm{p}\left(\mathrm{g}_{4} 2 \mathrm{~T}-\mathrm{T}\right)$ films and their mechanical properties. Doping of $\mathrm{p}\left(\mathrm{g}_{4} 2 \mathrm{~T}-\mathrm{T}\right)$ by TFSI from $0 \%$ to $10 \%$ gradually increases the $\pi-\pi$ stacking between polymers. It is also found that when doped by F4TCNQ, the elastic modulus and electrical conductivity of films increases until the doping level of about $18 \%$. We attribute these results to the increasing of $\pi-\pi$ stacking between inter-polymer backbones upon increasing the doping levels from $0 \%$ to $18 \%$.

Finally, the impact of the ratio of TVT/TET in pNDI-TVT - TET $_{1-\mathrm{x}}$ on the morphology and mechanical properties was studied. From MD simulations, we find that the $\pi$ - $\pi$ stacking between polymers as the TVT content increases till $50 \%$ and afterwards slightly decreases. In 
addition, a thin-film transistor with the TVT content of 60 or $80 \%$ shows a better conductivity than the one with $100 \%$ content when it is bent. Our findings on polymerization of conducting polymers, evolution of crystalline and mechanical properties provide theoretical insight that can help a practical improvement in the field of flexible organic electric devices. 


\section{Populärvetenskaplig Sammanfattning}

Numera används ledande polymerer i stor utsträckning för att utveckla lättviktiga, flexibla och biokompatibla elektriska enheter, allt från batterier och OLED-TV-apparater till bioelektronik. Som resultat har ledande polymerer under de senaste två decennierna varit ett område av stort intresse för både akademi och industri. Dock så är det fortfarande utmanande att utforma en ledande polymer som uppfyller nödvändiga elektriska och mekaniska egenskaper, eftersom dessa egenskaper samtidigt påverkas av många faktorer såsom laddningsbärarkoncentration, molekylvikt och kemisk struktur.

Beräkningsmetoder baserade på kvantmekanik och molekylär dynamik representerar viktiga verktyg i studier av ledande polymer eftersom de ger information om morfologin hos filmer på molekylär nivå och fysiska egenskaper så som termodynamisk potential, som experimentella studier kan förlita sig på. I denna avhandling utförde vi beräkningsstudier för polymerisation, kristallisering och för de mekaniska egenskaperna hos ledande polymerer (PEDOT, $\mathrm{p}\left(\mathrm{g}_{4} 2 \mathrm{~T}-\mathrm{T}\right)$ och pNDI-TVT-TET). Medans PEDOT och $\mathrm{p}\left(\mathrm{g}_{4} 2 \mathrm{~T}-\mathrm{T}\right)$ används vanligtvis som laddningsbärare transportörer för positivladdning, så kan pNDI-TVT-TET används som laddningsbärare transportör för både positiv och negativ laddning.

Först beräknar vi reaktionsenergin enligt en konventionell polymerisationsmekanism för PEDOT med Fe(TOS) 3 som oxidant för att teoretiskt bekräfta den. Vi visar att PEDOT med dopningsnivå högre än $33 \%$ blir energiskt ogynnsamt och förklarar det i termer av laddningslokalisering. Här betyder dopning kemisk oxidation av polymer genom oxidantmolekyl, vilket leder till polymerer med positiv laddning. För att undersöka effekten av polymerisationstemperatur på PEDOT-längden utvecklar vi en polymeriseringsmodell för samma polymerisationsmekanism. Resultaten visar att den genomsnittliga kedjelängden är längre vid högre temperaturer, vilket leder till slutsatsen att medellängden för PEDOT huvudsakligen begränsas av diffusiviteten hos reaktanter som också gradvis minskar under polymerisation på grund av kristallisation.

Studier om effekten av molekylär dopning på morfologin hos p(g $\left.\mathrm{g}_{4} 2 \mathrm{~T}-\mathrm{T}\right)$-filmer och deras mekaniska egenskaper, finner vi parallell stapling av inter-polymerhuvudkedjor som uppkommer från en attraktiv interaktion till att utvecklas som dopad av TFSI från 0\% till 10\%, vilket gör filmer mindre sköra än en vanlig $\mathrm{p}\left(\mathrm{g}_{4} 2 \mathrm{~T}-\mathrm{T}\right)$ film. Det har också visat sig att när den dopas av F4TCNQ ökar filmens elastiska modul och elektriska konduktivitet upp till dopningsnivåer på cirka 18\%. Vi hänför dessa resultat till ökningen av parallell stapling mellan inter-polymerhuvudkedjor vid ökning av dopningsnivåerna från $0 \%$ till $18 \%$.

Slutligen studeras effekten av innehållet av $\pi$-konjugerad TVT (ditienylvinylen) (eller icke $\pi$ konjugerad TET(ditienyletan)) i pNDI-TVT TET $_{1-x}$ på morfologin och mekaniska egenskaper. Vi finner att parallellstackningen mellan polymerer ökar när TVT-innehållet ökar upp till 50\% och därefter minskas något. Dessutom visar en tunnfilmstransistor med 60 eller $80 \%$ TVTinnehåll bättre konduktivitet än den med $100 \%$ innehåll när den är böjd. Våra resultat om 
polymerisation av ledande polymerer, utveckling av kristallina och mekaniska egenskaper ger teoretisk insikt som kan hjälpa en praktisk förbättring av flexibla organiska elektriska enheter. 


\section{Acknowledgements}

The four-year journey as a PhD student at Laboratory of Organic Electronics is an unforgettable experience in my life. This thesis has been possible by the contribution of many people. Therefore, I would like to express my gratitude to:

First, my main supervisor Igor Zozoulenko for giving me the opportunity to join the group and pursue my scientific interests. Thank you for your committed supervision and encouragement. Your enthusiasm for computational studies has always inspired me and guided my $\mathrm{PhD}$ course in the right direction.

My co-supervisor Xavier Crispin and Mathieu Linares for showing me a genuine attitude to research. Mathieu, thank you for the seminars about visualization and critical comment on my research.

My mentor Felipe for being my mentor and colleague. Felipe, thank you for teaching me your knowledge and tips as well as collaborations. I only wish I spent more time with you in Norrköping.

The members of Theory and Modelling for Organic electronics for providing a vibrant and inspiring working environment. My special thanks go to Ihor for your scientific advice, comment on the thesis draft and great Ukrainian foods. Aleksandar for your mentoring and commenting on the thesis draft. Sarbani for the fruitful collaboration. Najmeh D for the interesting chit-chat and discussion on research.

All administrators at LOE for sorting everything out.

All my external collaborators for great collaborations.

Other colleagues and friends that I have met at LOE and outside for a heartwarming environment and making my $\mathrm{PhD}$ course more exciting. Especially, I would like to thank Sämi, Lonni, Jacky, Evelina, Eva, Dennis, and Saumya for the great trip and all the fun. Jee-woong for all activities and a few words of encouragement. Evan for teaching me lots of experiments. Johannes $\mathrm{G}$ for commenting on the thesis draft and Pearl talks. Tobias for the amazing trip and support with the Swedish abstract. Gwennael for the interesting discussion on paintings in NY. Marie and Eric $\mathrm{G}$ for being good neighbors and tandem food. Tero and family for your hospitality. Chiara for the good Lasagna. Nadia and Daniel for all activities and bright vibes. Mehmet for the nice time in TD Garden, Boston. Yiannis for teaching me lots of PEDOT and the collaboration. Mina, Maria, Bernhard, Tran, Jennifer, Xenofon, Ulrika, Nara, Cyril, Vasileios, Arghyamalya, Benjamin, Giancarlo, Hamid, Arman, Roman, Astrid, David P, Laura, and Jonathan for nice fika, lunch, and evenings.

Finally, I would like to thank my family for their support and love. 


\section{List of Included Publications}

Paper I.

Why Is Pristine PEDOT Oxidized to 33\%? A Density Functional Theory Study of Oxidative Polymerization Mechanism

Donghyun Kim, Igor Zozoulenko

Journal of Physical Chemistry B, 2019, 123, 5160

Contribution: Carrying out simulations, Data analysis, Writing manuscript

Paper II.

How long are polymer chains in Poly(3,4- ethylenedioxythiophene):Tosylate Films? An insight from Molecular Dynamics Simulations

simulations

Donghyun Kim, Juan Felipe Franco-Gonzalez, Igor Zozoulenko

Submitted to Journal of Physical Chemistry B

Contribution: Developing a polymerization model with Juan Felipe-Gonzalez, Carrying out simulations, Data analysis, Writing manuscript

Paper III.

Influence of Acid Doping on the Nanostructure of a polythiophene with oligoether side chains

Sandra Hultmark, Furqan Farooqi, Donghyun Kim, Emmy Järsvall, Sara Marina, Jaime Martin, Igor Zozoulenko, Christian Müller

Manuscript in Preparation

Contribution: Carrying out MD simulation, Analysis of MD results, Writing MD part of manuscript

Paper IV.

Tuning of the elastic modulus of a soft polythiophene through molecular doping

Sepideh Zokaei, Donghyun Kim, Emmy Järsvall, Abigail Fenton, Albree Weisen, Sandra Hultmark, Phong Nguyen, Amanda Matheson, Anja Lund, Renee Kroon, Michael Chabinyc, Enrique Gomez, Igor Zozoulenko, Christian Müller

Submitted to Materials Horizons

Contribution: Carrying out MD simulation, Analysis of MD results, Writing MD part of manuscript

Paper V. 
Synergistic Mechanical-Electronic Naphthalenediimide Polymers with Tuned In-Chain $\pi$ Conjugation for Flexible Transistor

Dan Zhao, Donghyun Kim, Sarbani Ghosh, Gang Wang, Wei Huang, Junsheng Yu, Igor Zozoulenko, Tobin Marks, Antonio Facchetti

Manuscript in Preparation

Contribution: Carrying out Film deformation simulation, Data Analysis, Writing deformation simulation part of manuscript 


\section{Table of Contents}

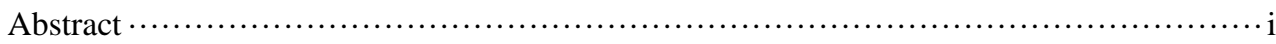

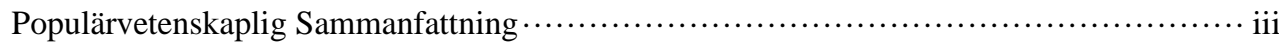

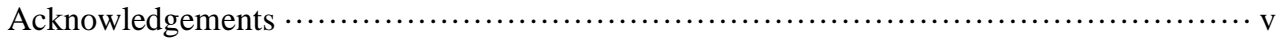

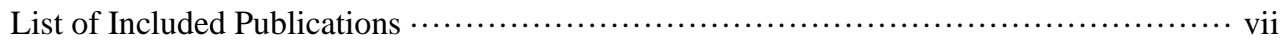

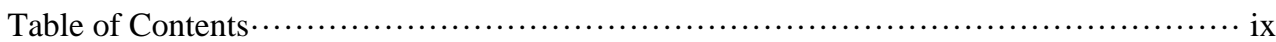

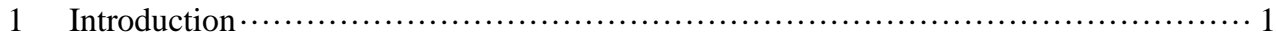

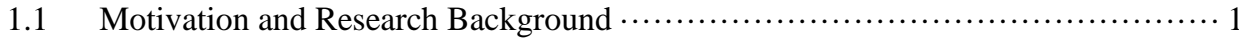

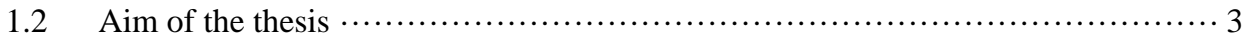

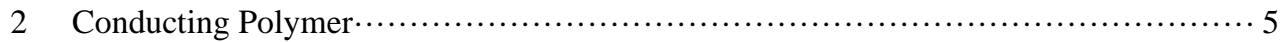

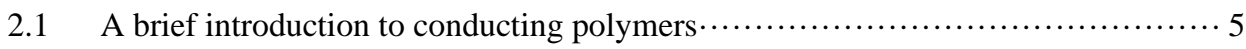

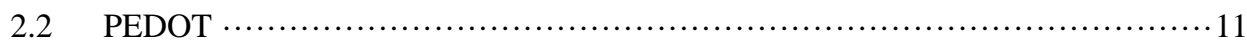

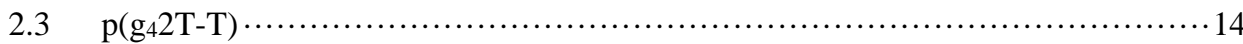

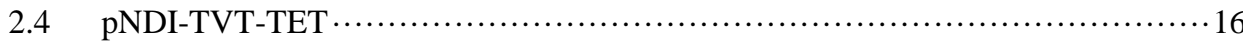

3 Mechanism of In-Situ chemical polymerization of PEDOT:TOS $\ldots \ldots \ldots \ldots \ldots \ldots \ldots \ldots \ldots \ldots \ldots \ldots \ldots$

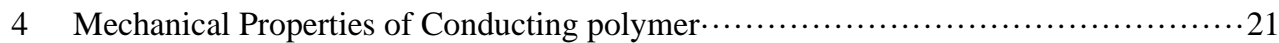

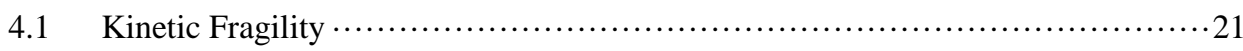

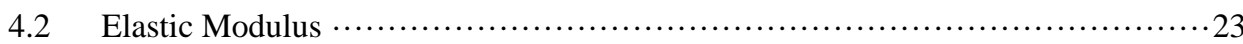

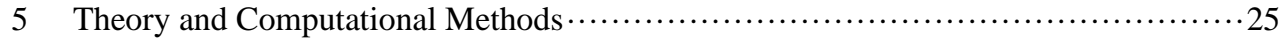

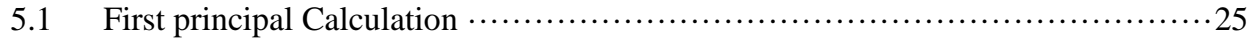

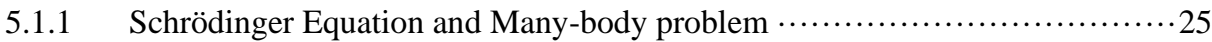

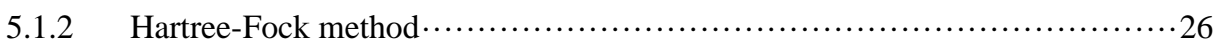

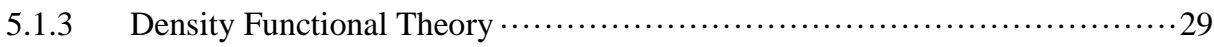

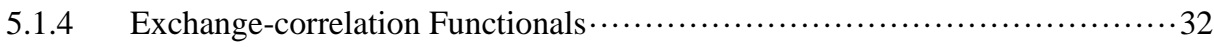

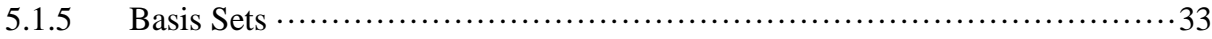

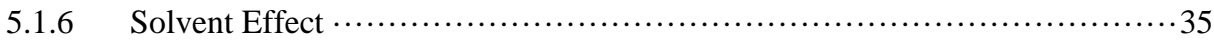

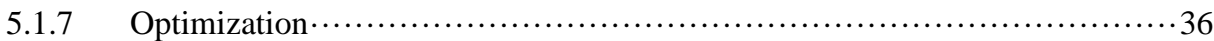

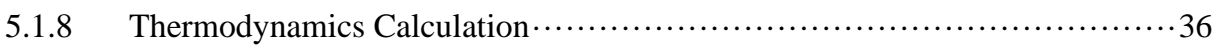

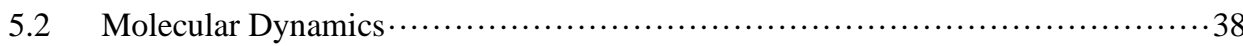

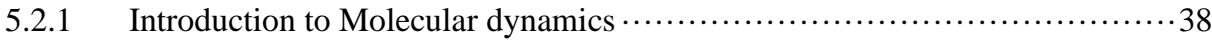

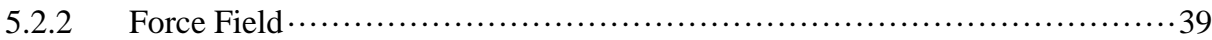

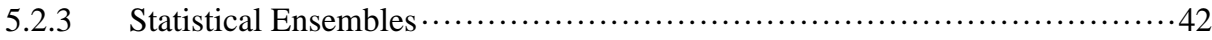




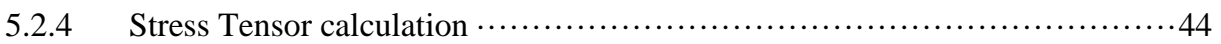

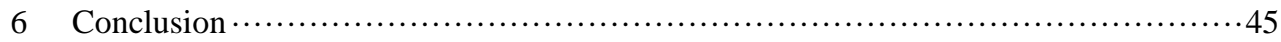

6.1 In-situ chemical polymerization mechanism and morphology of PEDOT:TOS $\cdots \cdots 45$

6.2 Molecular doping effect on the electrical and mechanical properties of $\mathrm{p}\left(\mathrm{g}_{4} 2 \mathrm{~T}-\mathrm{T}\right) \cdot 46$

6.3 Tuning the mechanical properties of pNDI-TVT-TET by controlling the $\pi$ -

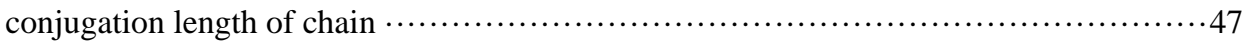

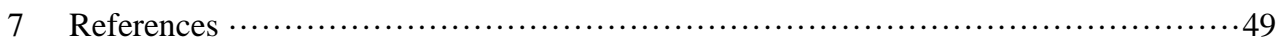

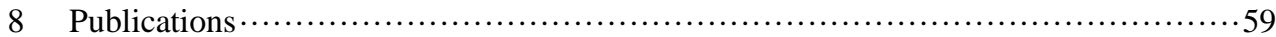




\section{Introduction}

\subsection{Motivation and Research Background}

It is almost impossible to maintain the contemporary lifestyle without the use of polymers. Even before the advent of the first synthetic polymer (Bakelite) in 1907, a variety of polymers from nature such as rubber, cellulose, starch and silk had been used in various applications as adhesives, packaging, textile and so on. Since the first synthetic polymer produced by Leo Baekeland (Bakelite), various synthetic polymers substituted not only natural polymers but also inorganic materials thanks to their light weight, great mechanical properties and capability for facile and economical mass-production. Some representative examples include plastic containers for fresh vegetables and meat (replacing containers of paper or glass), synthetic fibre such as polyester and nylon used for clothes, and engineering plastics like polyamide, polycarbonate for some metal parts of vehicles.

A development of polymers that is electrically conductive as metals gave rise to a new wave to our perspective on polymers. A discovery ${ }^{1,2}$ that Polyacetylene doped by Iodine vapor exhibited conductivity increased by over seven orders of magnitude compared to pristine Polyacetylene won Alan J. Heeger, Alan G.Macdiarmad, and Hideki Shirakawa Nobel Prize in Chemistry in 2000. ${ }^{3}$ This new discovery caused enormous interest for the use of polymers in electric devices such as Organic light emitting diode, Photovoltaic cells, transistor and in the field of bioelectronics. In addition to replacing inorganic materials in electric applications, conductive polymer opened up a new horizon due to the fact that polymers can be more flexible than inorganic materials, and therefore organic electric devices become stretchable, flexible or wearable.

In the light of the potential of conductive polymers, it seemed only a matter of time to make a breakthrough of organic electric devices. However, there were only a few technically practical applications that exploited conductive polymers due to its deficient properties, that is instability of conductive polymer in doped state and low conductivity. Thus, there have been intensive efforts for several decades aimed at finding new conductive polymers and understanding doping, conductivity, morphology, crystallinity, optical and mechanical properties via both theoretical and experimental methods.

Developments of synthesis method and deeper understanding of electrical and mechanical properties expanded the family of conductive polymers and improved their properties. Among various conductive polymers, Poly(3,4-ethylenedioxithiophene), also known as PEDOT, synthetized for the first time in 1988 by Bayer AGy, has gained lots of attention since it has high electronic and ionic conductivity and stability against air humidity, resulting in a boost in organic electric devices.

In addition to thiophene based polymers, naphthalenediimide (NDI) based polymers are also very promising conductive polymers that are widely used for organic field-effect transistor, thermoelectric generator, light emitting diode and photovoltaic cells. ${ }^{4-7}$ The attractive electrical properties of NDI based polymers is attributed to the fact that NDI could copolymerize with 
donor units (electron-rich) in which NDI unit is acceptor unit (electron-poor). Donor-Acceptor copolymers exhibit ambipolar charge transport that is capable of transporting electron and hole, making it possible to be utilized as n-type materials. Moreover, this donor-acceptor copolymers typically have a low bandgap arising from overlapping of $\mathrm{p}$ orbitals of donor and acceptor, leading to high conductivity. 


\subsection{Aim of the thesis}

Optimizing the conductivity and mechanical properties of conducting polymers is the key to the fabrication of flexible or wearable organic electric devices. To this end, it is necessary to understand charge carrier concentration and morphology of conducting polymers that are crucial factors affecting their mechanical and electrical properties. The studies in this thesis were focused on mainly two parts. One is theoretical understandings on the mechanism of PEDOT polymerization and its crystallization. The other is to explore mechanical properties of polythiophene with ethylene glycol side chains and NDI-based polymer.

Although a great deal of experimental and theoretical studies have been done to improve the performance and to understand the underlying knowledge of above properties, there are still lots of things on which the detailed knowledge is missing: 1) doping level and relatively short chain length of PEDOT that are important factors to the electrical conductivity, 2) molecular doping effect on the morphology of $\mathrm{p}\left(\mathrm{g}_{4} 2 \mathrm{~T}-\mathrm{T}\right)$ as well as its mechanical properties, 3$)$ how the $\pi$-conjugation length of $\mathrm{p}\left(\mathrm{NDI}_{-} \mathrm{TVT}_{\mathrm{x}}-\mathrm{TET}_{1-\mathrm{x}}\right)$ controlled by incorporation of $\pi$-nonconjugated unit (TET) affects the morphology as well as the mechanical properties of the thin films when used for a flexible organic field effect transistor.

In paper I, In-situ oxidative chemical polymerization (ICP) mechanism of PEDOT:TOS was corroborated as well as $33 \%$ of doping level of pristine PEDOT was explained by using Density Functional Theory to calculate the Gibbs Free energy evolution as per conventional polymerization and doping steps. Based on the insights from paper I, a polymerization model using Molecular Dynamics for ICP of PEDOT:TOS was introduced to study the effect of temperature on PEDOT polymerization as reported in paper II.

In paper III and IV, how molecular doping affects the morphology (paper III and IV) and elastic modulus (paper IV) was investigated by MD simulations together with experiments. In paper $\mathrm{V}$, the morphology and elastic modulus of pNDI-TVT ${ }_{\mathrm{x}}-\mathrm{TET}_{1-\mathrm{x}}$ with a different ratio of $\pi$ conjugated unit (TVT) to $\pi$-nonconjugated unit (TET) in chains were studied using MD simulations. With an optimal ratio, a flexible organic thin-film transistor (FOTFTs) was fabricated, and its electrical properties and flexibility were assessed by a bending test.

In paper I-IV, I conducted Density Functional Theory (DFT) and Molecular Dynamics (MD) calculations, analyzed the computed data and wrote manuscripts. In paper II, I developed a polymerization model with classical MD. In paper V, I contributed to the MD simulations concerning the film deformation simulations as well as analyzing data and writing manuscript.

The aim of this thesis is to provide fundamental and practical knowledge on the mechanism of polymerization and crystallization of PEDOT:TOS as well as mechanical properties of conducting polymers. Chapter 2 gives an introduction to conducting polymers as well as the materials studied in this thesis. The mechanism of In-situ oxidative chemical polymerization for PEDOT:TOS for the studies related to paper I and II is elaborated in Chapter 3. Chapter 4 describes the mechanical properties of conducting polymers such as kinetic fragility and elastic 
modulus. Chapter 5 provides an overview of computational approaches employed for the studies. Finally, Chapter 6 summarizes conclusions of the thesis and outline of the publications. 


\section{Conducting Polymer}

\subsection{A brief introduction to conducting polymers}

The word "polymer" originates from two Greek words, 'poly' and 'mer' that literally means 'many' and 'part' respectively. As the etymology of polymer implies, polymers consist of many repeating units, representing one kind of monomer or different kinds of monomer, i.e. copolymer. Before the discovery of conducting polymers, other properties of polymers such as light weight, mechanical flexibility and electrical insulation were primarily considered when utilizing polymers for applications.

In the history of conducting polymers, the discovery of electrical conductivity of Polyacetylene (Figure 1a) in the 1970s led to the considerable interest about conducting polymers. On the other hand, there had been studies about the nature of electrical conductance of polymer that predated Polyacetylene. The first documented report of conducting polymer was Polypyrrole, Figure 1b, studied by Don Weiss in 1963. ${ }^{8-10}$ Even before the study of Polypyrrole, Polyaniline (Figure 1c) has been used for about 150 years particularly for indigo dyes ${ }^{11}$ until its electrical conductivity was reported for the first time by Jozefowicz in $1967 .{ }^{12}$ Polythiophene (Figure 1d) was polymerized in the 1930s, but its electrical conductivity was overlooked until its remarkable conductivity, $10 \sim 100 \mathrm{~S} / \mathrm{cm}$, when doped by Tetrabutylammonium tetrafluoroborate $\left(\mathrm{N}(\mathrm{Bu})_{4} \mathrm{BF}_{4}\right)$ was reported by Tourillon and Garnier ${ }^{13}$ in 1982, while its potential electrical conductivity was mentioned by Armour et al in 1967. ${ }^{14}$

(a)

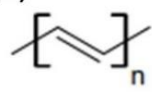

(b)

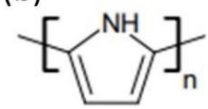

(c)

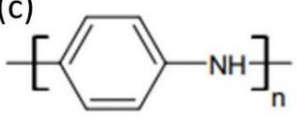

(d)

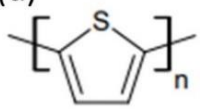

Figure 1 Structure of conductive polymers: a) Polyacetylene b) Polypyrrole

c) Polyaniline d) Polythiophene

Where does the electrical conductivity come from? The answer is doped polymers with a conjugated system in their backbone that is alternation of single bond and double bond between carbon atoms. Explaining the structure of the conjugated system, this arises from the hybridization of valence orbitals of carbon atoms which have an electronic configuration $\left(1 s^{2} 2 s^{2} 2 p^{2}\right)$ in the ground state. As illustrated in Figure 2, the valence shell orbitals ( $2 s$ and $\left.2 p\right)$ of Carbon atom form hybridized orbitals by combining 2 s orbital with 1 to 3 of $2 p$ orbitals, resulting in $\mathrm{sp}, \mathrm{sp}^{2}, \mathrm{sp}^{3}$ orbitals respectively.

The $\mathrm{sp}^{2}$ hybridization is of interest for conducting polymers. Let's see the simplest case, i.e. Ethene $\left(\mathrm{C}_{2} \mathrm{H}_{4}\right)$ backbone of which consists of two carbon atoms with $\mathrm{sp}^{2}$ hybrid orbitals. In this case, two carbon atoms make a $\sigma$ bond using a $\mathrm{sp}^{2}$ orbital in each carbon atom as well as a $\pi$ bond between $2 \mathrm{p}_{\mathrm{z}}$ orbitals of carbon atoms as illustrated in Figure $3 \mathrm{a}$, resulting in double bond between two carbon atoms. Please note that $\sigma$ and $\pi$ orbitals are bonding molecular orbitals whereas $\sigma^{*}$ and $\pi^{*}$ orbitals are antibonding molecular orbitals. The four remaining $\mathrm{sp}^{2}$ orbitals of carbons in Ethene form bonds with s orbitals of 4 hydrogens. In Ethene, HOMO (Highest 
Occupied Molecular Orbital) and LUMO (Lowest Unoccupied Molecular Orbital) are $\pi$ and $\pi^{*}$ orbital, respectively.

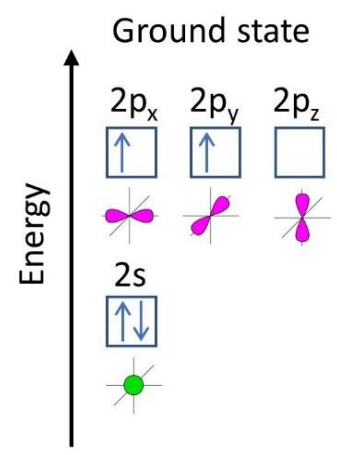

Excited state

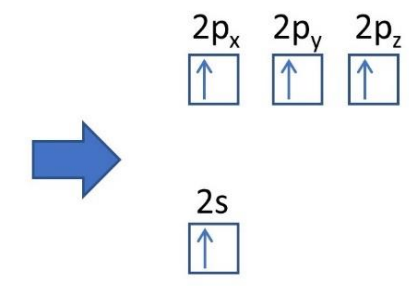

Hybridization

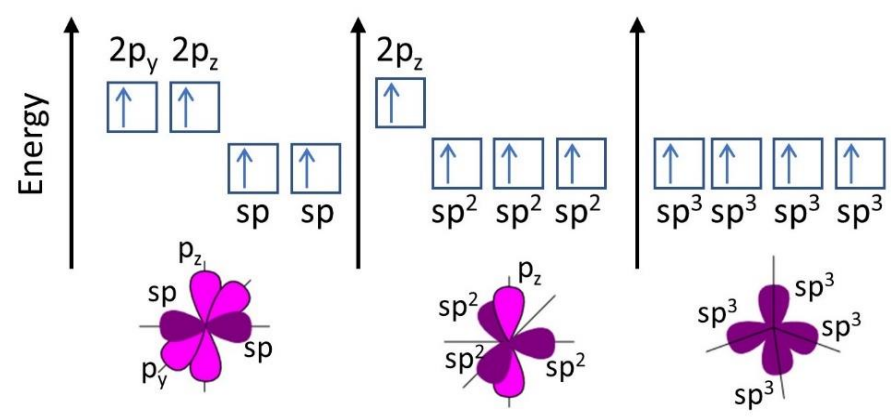

Figure 2 Orbital Hybridization of Carbon atom; resulting electron configuration and shape of hybrid orbitals for $s p, s p^{2}$, and $s p^{3}$ hybridization

For a conjugated polymer that has a longer conjugated system in the backbone than Ethene, overlapping $2 \mathrm{p}_{\mathrm{z}}$ orbitals in carbon atoms results in a set of $\pi$ orbitals (as well as a set of $\pi^{*}$ orbitals) delocalized throughout the backbone that have close energy level as can be shown in Figure 3b. The difference of energy level between $\pi$ orbitals (or $\pi^{*}$ orbitals) becomes infinitesimal as the length of conjugation increases infinitely, leading to two continuous energy bands comprising $\pi$ orbitals or $\pi^{*}$ orbitals that are comparable to valence and conduction bands of semiconductors, respectively. As $\pi$ orbitals are delocalized along the backbone, the electrons in $\pi$ band are relatively free to move. Note that $\sigma$ and $\sigma^{*}$ bands are also formed by overlapping $\mathrm{sp}^{2}$ orbitals, but they are less important in the electrical properties of conducting polymers since their energy level is lower (or higher) than that of $\pi$ band (or $\pi^{*}$ band). 
(a)

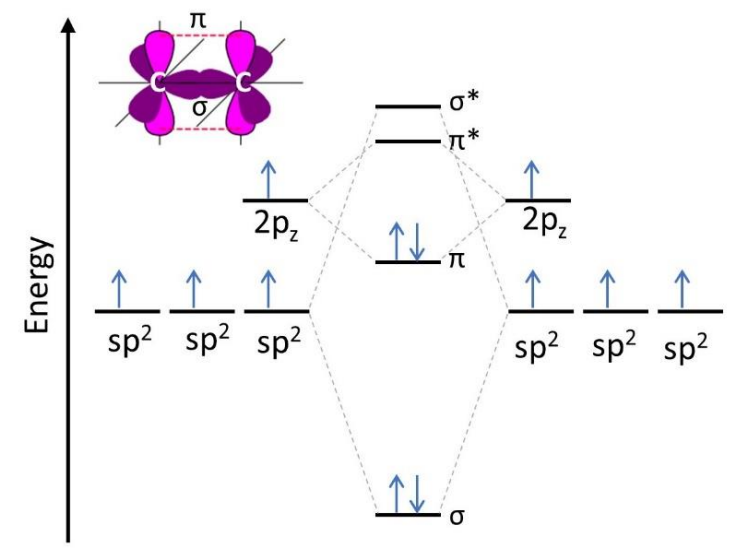

(b)

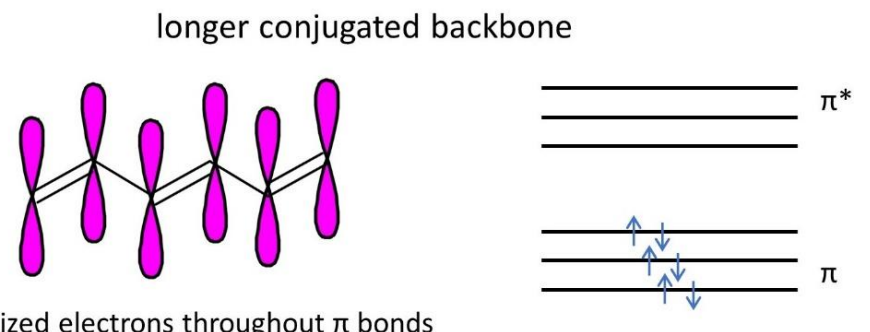

Delocalized electrons throughout $\pi$ bonds

Figure 3 (a) Molecular orbitals formed by two carbon atoms in Ethene. (b) $\pi$ and $\pi^{*}$ bands of conjugated polymer (Hexadiene). Note that $\sigma$ bonds between carbon $\left(s p^{2}\right.$ orbital) and hydrogen (1s orbital) are not described here. Neither are $\sigma$ and $\sigma^{*}$ band between carbon

Although the electrons in the delocalized $\pi$ band of conjugated polymers have relatively high mobility, most neutral (undoped) conjugated polymers are known to have very low electric conductivity because of their band gap $(1-4 \mathrm{eV})$ which is hard to overcome by thermal agitation. The reason that conducting polymers have such band gap as semiconductors is a distortion of the backbone caused by Peierls instability. ${ }^{3,15}$ This instability leads to a decrease in symmetry of the chain, resulting in the alternation of double and single bond and also a large band gap as semiconductors rather than a same bond length between carbon atoms in the backbone with a half-filled continuous band.

Doping introduces charges to conjugated polymers such that they become highly conductive $^{3,16,17}$, i.e. conductor. Doping can be done by either (chemical or electrochemical) reduction or oxidation, which induces negative (electron) or positive (hole) charges, resulting in n- or p-type materials.

In p-type doping, a dopant extracts an electron from HOMO of valence band (Oxidation) of a conducting polymer, introducing a quasiparticle, also called as (positive) polaron, with a 
positive charge $(\mathrm{Q}=+1)$. Removing one more electron from the conducting polymer results in either a polaron pair or bipolaron that is a quasiparticle with two positive charges $(\mathrm{Q}=+2)$. A formation of bipolaron rather than a polaron pair could arise when the energy gain from electron-lattice interaction is greater than the Coulombic repulsion between two holes. ${ }^{18}$ In pdoping, the positive charges of p-type conducting polymers is balanced by dopants that are negatively charged by electrons from oxidation of polymer chains.

According to findings by DFT studies on the electronic structure of Thiophene-based polymers, a polaron pair is energetically favorable for the ground state of $Q=+2$ when the chain length is longer than 6 compared to bipolaron. ${ }^{19-21}$ In addition, the location of counter anions would lead to the different ground state of $\mathrm{Q}=+2$ where bipolaron ground state is more preferable than a polaron pair when the two counter anions are close to each other, helping to overcomes Coulombic repulsion of two positive charges. Furthermore, it is even feasible that polaron and bipolaron coexist at higher doping level. Another feature of p-doping that should be noted is a change in electronic structure. The DFT studies demonstrate that the band structures of p-doped polymers differ from a traditional picture obtained from pre-DFT approches ${ }^{22-24}$ that predict a half-filled spin-degenerate orbital in the bandgap for polaron and an unoccupied spindegenerate orbital for bipolaron.

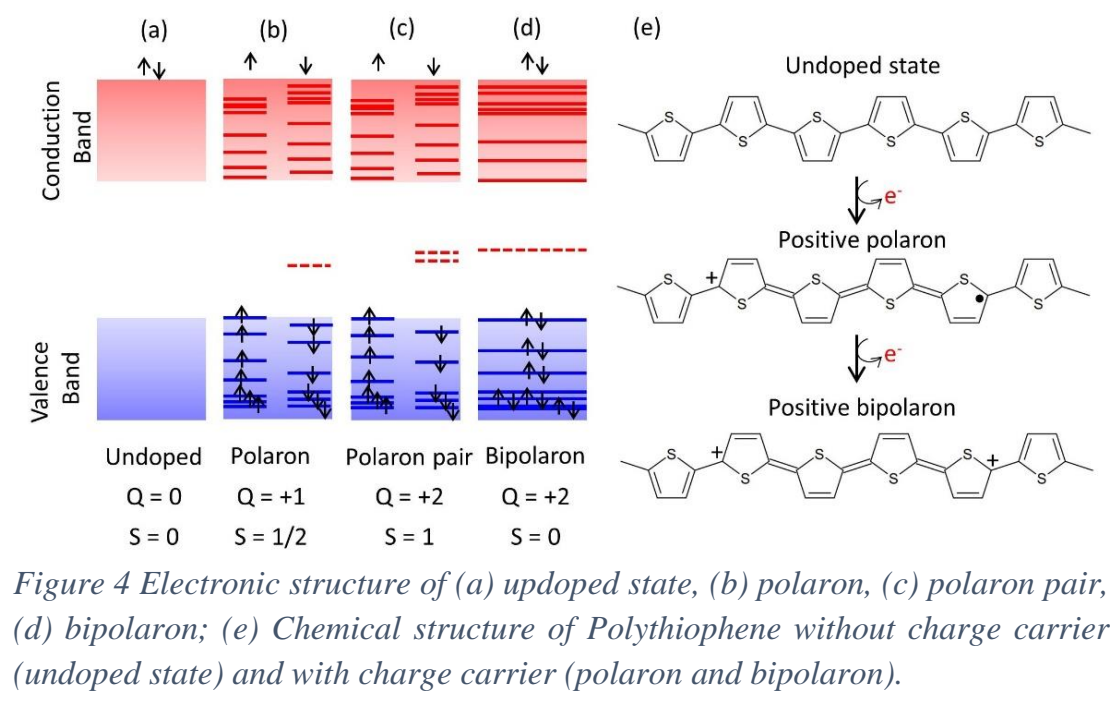

Compared to undoped state (Figure 4a), polaron exhibits an unoccupied spin-resolved orbital in the bandgap (between valence and conduction band), see Figure $4 \mathrm{~b}$. In the case of $\mathrm{Q}=+2$, as described above, it is possible to have either a polaron pair or bipolaron state. The polaron pair has two unoccupied orbitals with the same spin (spin-resolved) in the bandgap (Figure 4c), while bipolaron has an empty spin-degenerate orbital in the bandgap (Figure 4d). As a result of the change in electronic structure, the spin-multiplicity of polaron, polaron pair, and bipolaron state correspond to doublet $(S=1 / 2)$, triplet $(S=1)$, and singlet $(S=0)$ respectively. These quasiparticles, polaron and bipolaron, also induce distortion to the chemical structure of polymers. Figure $4 \mathrm{e}$ shows the chemical structure of polythiophene as it is oxidized from 
undoped state to bipolaron $(\mathrm{Q}=+2)$. Please note that the chemical structure of a chain with polaron pair is omitted since it is repetition of the structure for a polaron since a polaron pair means that two polarons are spatially separated in a chain.

In the case of n-type doping, the change in electronic structure and introduction of quasiparticles (negative polaron or bipolaron) to conducting polymers are similar with p-type doping, but the negative charge carriers (electron) are transferred from dopants to polymers (Reduction), occupying new orbitals in the bandgap. The electron structure for negative polaron has a half-filled spin-resolved orbital (doublet, $S=1 / 2$ ) while bipolaron shows a filled spin-degenerate orbital (singlet, $\mathrm{S}=0$ ). ${ }^{25}$

Whether it is p-type or n-type conducting polymer, the formation of quasiparticles (polarons or bipolarons) localized in the backbone of conducting polymers results in orbitals in the bandgap, leading to significant increase in conductivity, see Figure 4. It should be noted that a degenerate polymer like trans-polyacetylene possesses two identical structures in the ground state and thus solitons, neutral/positive/negative soliton, could also be charge carriers for transpolyacetylene. ${ }^{3,26,27}$ But in this thesis, non-degenerate polymers, such as PEDOT, p(g $\left.2 \mathrm{~T}-\mathrm{T}\right)$, and NDI-based polymer described in following chapters, are of our interest in which polaron or bipolaron serve as charge carrier.

Although doping extensively increases the mobility of charge carriers in conducting polymer, the mobility of charge carriers via inter-chain plays no less important role in the conductivity than the charge mobility via intra-chain. Polymer films comprise ordered crystalline and disordered amorphous regions. The $\pi$-conjugated system of conducting polymers allows $\pi$ - $\pi$ interaction (non-covalent interaction) between backbones in different chains, resulting in interplanar stacking between chains, best known as $\pi-\pi$ stacking, see Figure 5. Many studies have shown that the morphology of conducting polymer films plays a critical role in the nature of electrical conductivity, particularly such as crystallinity and effective $\pi-\pi$ stacking network where charge carriers could travel via $\pi-\pi$ stacked polymers. ${ }^{28-31}$ Therefore, on top of the molecular structure of conducting polymers, conducting polymer films need to have a welltuned morphology with an effective percolation path to improve their electrical conductivity. 


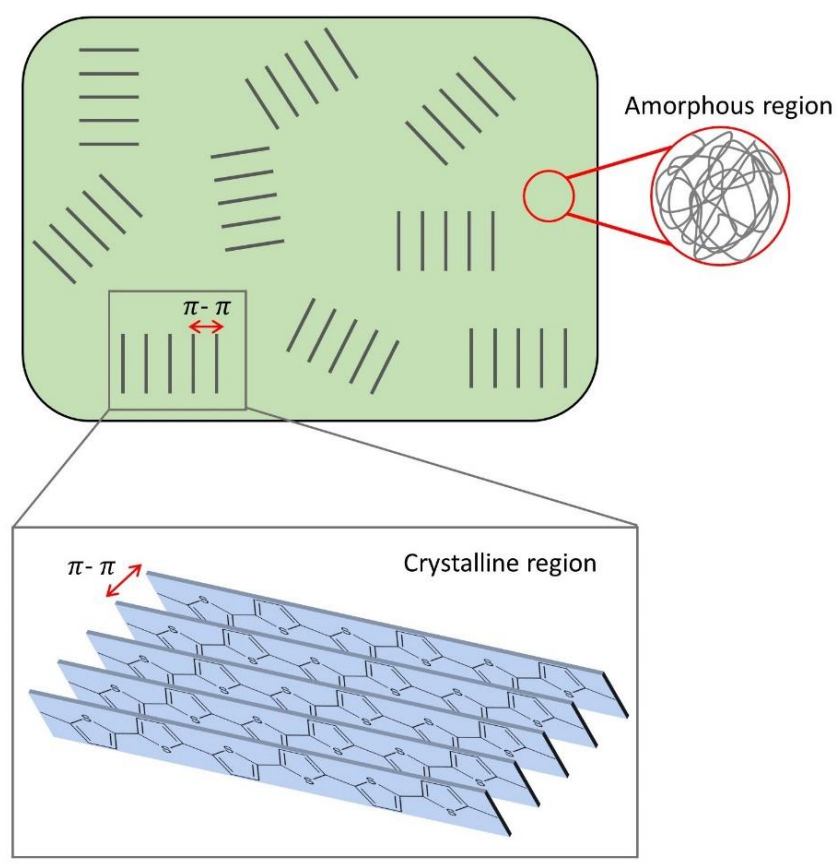

Figure 5 Conceptual Scheme for the morphology of conducting polymer consisting of Amorphous region and Crystalline region. 


\subsection{PEDOT}

Since the advent of conductive polyacetylene, immense efforts had been put into utilizing conducting polymers for commercial applications. A number of new conducting polymers, however, failed to achieve the goal mainly due to their instability in highly doped state (e.g. polyacetylene and polythiophene) and intrinsic drawbacks such as toxicity, intense color and poor transparency (e.g. polypyrrole). In 1988, PEDOT (Poly(3,4-ethylenedioxithiophene)), a thiophene derivative with bicyclic ring system, was invented by F. Jonas and G. Heywang. ${ }^{32-}$ 34

PEDOT manifests not only very high electronic (and ionic) conductivity up to $8797^{35} \mathrm{Scm}^{-1}$ but also outstanding stability in highly doped state (p-type) against air humidity and acid condition that are indispensable features for practical electric applications. ${ }^{36,37}$ The stability of PEDOT is attributed to the positive mesomeric effect of ethylenedioxy group that stabilizes the positive charges of oxidized PEDOT. ${ }^{34,38}$ In addition, a number of well-established polymerization methods make PEDOT attractive such as Vapor Phase Polymerization (VPP) ${ }^{39-41}$ Chemical Vapor Deposition (CVD),${ }^{42}$ Electrochemical Polymerization, ${ }^{43,44}$ and Insitu Chemical Polymerization. ${ }^{29,45,46}$ Owing to these superior properties and facile polymerization methods, PEDOT is used for a range of organic electric devices such as supercapacitors, ${ }^{47-50}$ ion-pumps, ${ }^{51,52}$ thermoelectric modulus, ${ }^{43,53-55}$ solar cells, ${ }^{56,57}$ fuel cells,${ }^{58-60}$ electrochromic display, ${ }^{61}$ light-emit diodes,${ }^{62}$ field effect transistors ${ }^{63-65}$ and devices for bioelectronics. ${ }^{45}$

A neutral PEDOT can have two different alternated structures that are aromatic and quinoid (see Figure 6), meaning that PEDOT has non-degenerate system as most other conducting polymers such as Polythiophene, Polypyrrole, and Polyaniline. Several computational studies found that a neutral PEDOT has aromatic-like structure in the ground state. ${ }^{19,66}$ On the other hand, (Trans-) Polyacetylene is a typical degenerate conducting polymer as aforementioned in the previous chapter.

(a)

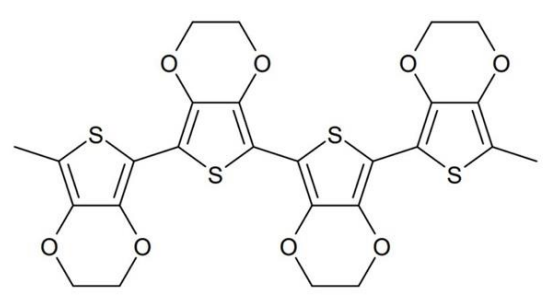

aromatic (b)

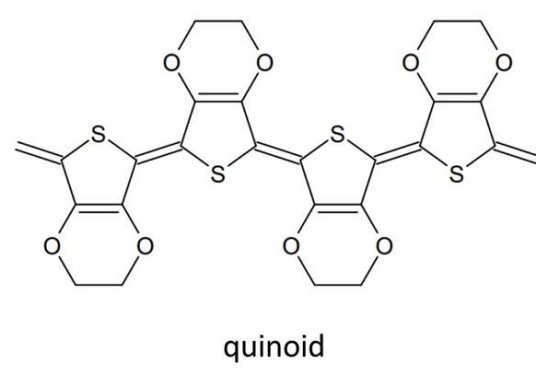

Figure 6 Chemical structures of neutral PEDOT (a) aromatic (b) quinoid

It is known that PEDOT has a doping level of $33 \%$ as polymerized (pristine PEDOT) via oxidative polymerization condition with the use of a variety of oxidants such as Sodium peroxodisulfate $\left(\mathrm{Na}_{2} \mathrm{SO}_{4}\right)$, Iron(III) Chloride $\left(\mathrm{FeCl}_{3}\right)$, Iron(III) Tosylate $\left(\mathrm{FeTOS}_{3}\right)$ and Iron(III) 
trifluoromethanesulfonate $\left(\mathrm{Fe}(\mathrm{OTf})_{3}\right)$. These oxidants also work as dopants when doping resulting oligomers to p-doped PEDOT. The positive charges of doped PEDOT are compensated by counter anions ranging from polymeric to molecular counter anions.

Polystyrene sulfonate (PSS) is one of the most popular counter anions, where deprotonated sulfonyl groups have negative charges, see Figure 7a. PEDOT:PSS dispersed in water is so stable that water-dispersion process is possible. However an excess of PSS in a PEDOT:PSS films could decrease the conductivity significantly since PEDOT:PSS could form core-shell structure where PEDOT-rich core is wrapped by PSS-rich shell, preventing PEDOT-rich regions from building a good $\pi-\pi$ network in the films.${ }^{67,68}$ Fortunately, various post-treatment could increase the conductivity of PEDOT:PSS by several orders of magnitude from $1 \mathrm{Scm}^{-1}$ up to about $4400 \mathrm{Scm}^{-1}$. $^{6,69}$

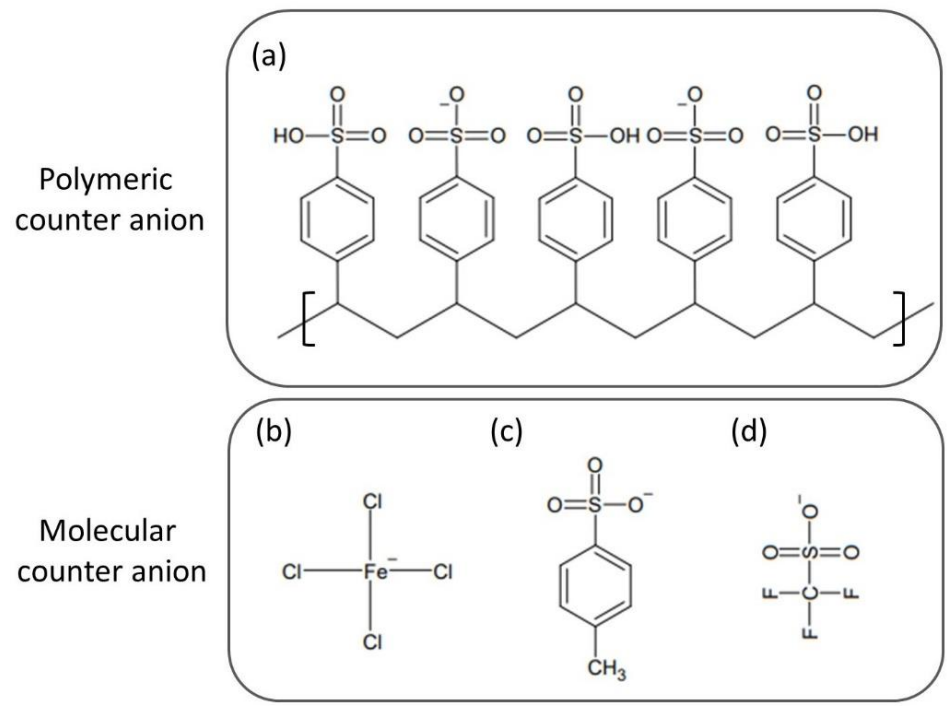

Figure 7 Chemical Structure of polymeric counter anion (a) PSS and molecular counter anions (b) $\mathrm{FeCl}_{4}^{-},(c) \mathrm{TOS}_{-}^{-}$, (d) OTf

Apart from polymeric counter anions, molecular anions such as $\mathrm{FeCl}_{4}^{-}$, $\mathrm{TOS}^{-}$, and $\mathrm{OTf}^{-}$(Figure $7 \mathrm{~b}-\mathrm{d}$ ) could be used as counter anions. PSS is merely counter anion which means that oxidant is needed to polymerize PEDOT, while molecular counter anions are concomitant molecules of oxidation of PEDOT by corresponding dopants (e.g. PEDOT $+\mathrm{FeTOS}_{3} \rightarrow \mathrm{PEDOT}^{+}+\mathrm{TOS}^{-}$ + FeTOS $_{2}$, Details of polymerization mechanism for PEDOT:TOS will be explained in chapter

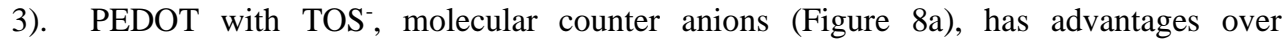
PEDOT:PSS that is higher electrical conductivity (up to circa. $8800 \mathrm{Scm}^{-1}$ when FeTOS 3 used as oxidant and dopant) as well as semi-metallic behaviour. ${ }^{70}$

Experimental and Computational studies have investigated the structure of crystallite of PEDOT:TOS film. ${ }^{70-74}$ GIWAXS characterization of PEDOT:TOS film demonstrated diffraction peaks at $\mathrm{Q}=0.45,0.89$, and $1.82 \AA^{-1}$ (lattice spacing, $\mathrm{d}=14,7.9$, and $3.4 \AA$ respectively) that are considered to come from (100), (200), and (010) planes of the film, respectively where (100) and (200) planes correspond lamellar stacking and (010) plane 
indicates $\pi$ - $\pi$ stacking between PEDOT chains. ${ }^{70,71}$ Figure $8 \mathrm{~b}$ depicts the lattice parameter of a crystallite of PEDOT:TOS that is $14,3.4$ and $7.9 \AA$ corresponding to lamellar stacking distance, $\pi-\pi$ stacking distance between PEDOT chains, and the distance of two repeating EDOT units, respectively.

(a)

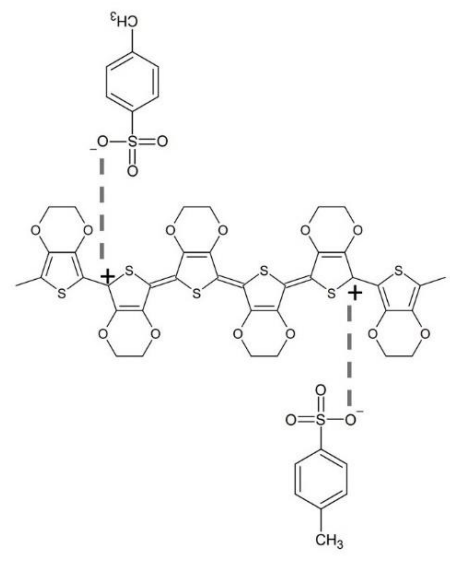

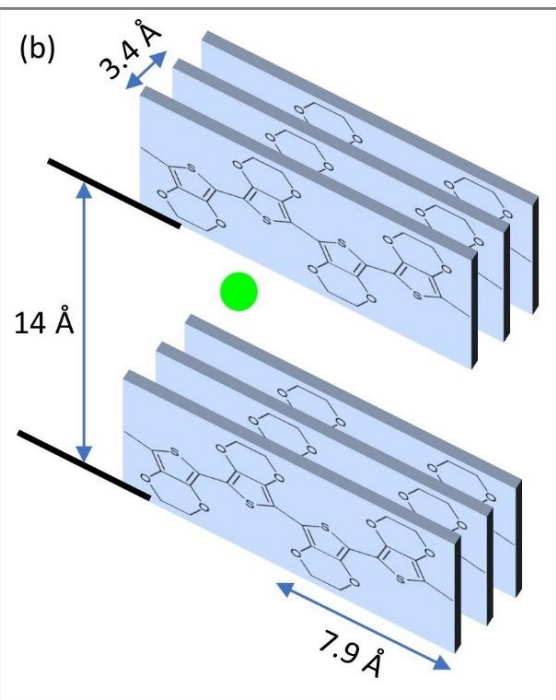

Figure 8 (a) PEDOT with two positive charges balanced by TOS- counter anions, (b) structure of PEDOT:TOS crystallite with lattice parameter where a TOS is represented in a green sphere. 


\section{$2.3 p\left(g_{4} 2 T-T\right)$}

Thiophene-based polymers decorated by oligo ethylene glycol (EG) side chains demonstrate electronic (and ionic) conductivity, thermal stability, and softness. In addition, it is possible to synthesize them via one-pot process (described in more depth later), enabling large-scale production at low cost. This class of polymers is, therefore, widely employed in organic electrochemical transistor (OECT) and bioelectronic applications. ${ }^{75-78}$ Since these properties have something to do with EG side chain, they could be engineered by changing the composition; for example, by the number of repeat EG units or the number of blocks. ${ }^{77}$

Polythiophene with EG side chain exhibits electronic and ionic conductivity. Some studies found that solvent uptake occurs simultaneously with ion transport facilitated by EG side chains, inducing volume expansion of more than $100 \% .^{76,77,79}$ On top of the surpassing volume expansion compared to other polymers, it is viable to control the volume of Polythiophene with EG side chain reversibly by electrochemical doping, and the volume change capability could be engineered by changing the length of the side chains. This reversible volume change by electrochemical doping enables the development of outstanding actuators.

Figure 9a shows the chemical structure of $\mathrm{p}\left(\mathrm{g}_{4} 2 \mathrm{~T}-\mathrm{T}\right)$ in which as the name indicates, a monomer comprises two thiophene rings (2T) decorated by 4 EG repeat units $\left(\mathrm{g}_{4}\right)$ and a thiophene ring (-T) with no EG side chain. As the $\pi$-conjugated system of $\mathrm{p}\left(\mathrm{g}_{4} 2 \mathrm{~T}-\mathrm{T}\right)$ is derived from Polythiophene, it has similar properties to Polythiophene which is usually p-type conducting with polarons and bipolarons as charge carrier. $\mathrm{p}\left(\mathrm{g}_{4} 2 \mathrm{~T}-\mathrm{T}\right)$ is extremely soft due to a low glass transition temperature $\left(\mathrm{Tg} \approx-46^{\circ} \mathrm{C}\right)$ and exhibits low crystallinity compared to P3HT (Tg $\approx 23$ ${ }^{\circ} \mathrm{C}$, when regioregularity $>97 \%$ ) that has a similar chemical structure but substituted by an aliphatic side chain (Hexyl group).

(a)

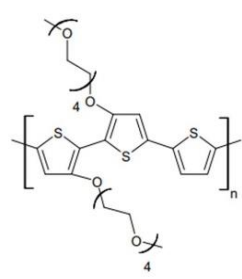

$\mathrm{p}\left(\mathrm{g}_{4} 2 \mathrm{~T}-\mathrm{T}\right)$ (b)

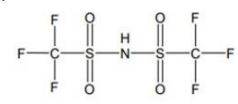

TFSI

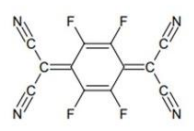

F4TCNO
(C)

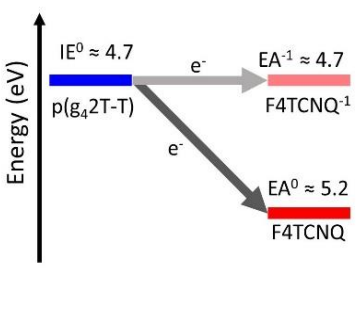

Figure 9 Chemical Structure of $p\left(g_{4} 2 T-T\right)$ and (b) dopants (TFSI and F4TCNQ); (c) Energy diagram for double doping by F4TCNQ with Ionization Energy of $p\left(g_{4} 2 T-T\right)$ and Electron Affinity of F4TCNQ

On the one hand, most other conducting polymers such as P3HT and PEDOT are stiff and need to be softened for flexible electric devices. On the other hand, considerable softness of $\mathrm{p}\left(\mathrm{g}_{4} 2 \mathrm{~T}-\right.$ T) makes it challenging to make practical electric devices from this polymer. Therefore, $\mathrm{p}\left(\mathrm{g}_{4} 2 \mathrm{~T}-\mathrm{T}\right)$ should be more ductile and stiff to the point where it becomes free-standing solid by 
copolymerization with hard urethane segment, ${ }^{80}$ while other stiffer conducting polymers are softened by adding plasticizers ${ }^{55}$ or copolymerization with soft block.

In addition, doping $\mathrm{p}\left(\mathrm{g}_{4} 2 \mathrm{~T}-\mathrm{T}\right)$ by oxidative dopants such as p-toluensulfoic acid (pTSA), trifluoromethanesulfonimide (TFSI) and 2,3,5,6-tetrafluoro-7,7,8,8-tetracyanoquinodimethane (F4TCNQ) is found to increase the crystallinity and conductivity. ${ }^{81}$ Figure $9 \mathrm{~b}$ illustrate the chemical structure of TFSI and F4TCNQ that are used in paper III and IV respectively.

X-ray scattering experiments disclosed the morphology of $\mathrm{p}\left(\mathrm{g}_{4} 2 \mathrm{~T}-\mathrm{T}\right)$ where sharp peaks are observed at $\mathrm{Q} \approx 0.36$ and $0.71 \AA^{-1}$ (lattice spacing $\mathrm{d} \approx 18$ and $8.8 \AA$ ) respectively corresponding to (100) and (200) planes of lamellar stacking, while a peak for (010) plane that indicates $\pi-\pi$ stacking between polymer chains is absent when doping level is $0 \%{ }^{80}$

Since EG side chains increase the solubility of $\mathrm{p}\left(\mathrm{g}_{4} 2 \mathrm{~T}-\mathrm{T}\right)$ polymers in more polar solvents such as acetonitirile and DMF (dimethylformamide), a polar molecular dopant like F4TCNQ (Figure 9b) could have higher solubility. This good solubility allows a simple one-pot process with mixed solution which includes polymer and dopants. On the other hand, Polythiophene with aliphatic side chain, for example P3HT, requires a sequential doping process to prevent doped polymers from aggregation, leading to considerable decrease in conductivity. ${ }^{81,82}$ Moreover, EG side chains suppress the sublimation of F4TCNQ from films, leading to enhanced thermal stability. ${ }^{83}$

F4TCNQ as dopant for $\mathrm{p}\left(\mathrm{g}_{4} 2 \mathrm{~T}-\mathrm{T}\right)$ has another attractive feature that is double doping. This means that $\mathrm{p}\left(\mathrm{g}_{4} 2 \mathrm{~T}-\mathrm{T}\right)$ could be oxidized (doped) by neutral F4TCNQ as well as F4TCNQ anion, leading to a higher ionization efficiency than $100 \%$ (for the case of single doping). This higher ionization efficiency by double doping is attributed to the lower ionization energy (IE) of $\mathrm{p}\left(\mathrm{g}_{4} 2 \mathrm{~T}-\mathrm{T}\right)\left(\mathrm{IE}^{0} \approx 4.7 \mathrm{eV}\right)^{80}$ than the electron affinity (EA) of neutral F4TCNQ and as big as the EA of F4TCNQ anion $\left(\mathrm{EA}_{\mathrm{F} 4 \mathrm{TCNQ}}^{0} \approx 5.2 \mathrm{eV}, \mathrm{EA}_{\mathrm{F} 4 \mathrm{TCNQ}^{-1}}^{-1} \approx 4.7 \mathrm{eV}\right)^{84}$, as described in Figure 9c. 


\section{4 pNDI-TVT-TET}

NaphthaleneDiimide (NDI)-based polymers are an attractive class of conducting polymers because it could be a n-type conducting polymer that is necessary to realize an organic field effect transistor and organic thermoelectric generator. Despite tremendous achievements in ptype materials such as Polythiophene-based polymers, n-type conducting polymers need improvement in terms of stability in doped state and conductivity. To this end, engineering main chain and side chain has been investigated. The backbone of the main chain could be engineered by chemical modification and copolymerization. ${ }^{85-87}$ On the other hand, side chain could be modified by choosing side chains with different length, chemical structure and polarity, imparting steric hindrance, polarity and rigidity. $7,84,88,89$

pNDI-TVT-TET is one of NDI-based copolymers with $\pi$-conjugated unit (TVT, dithienvinylene) or $\pi$ - nonconjugated unit (TET, dithienylethane), see Figure 10a. Furthermore, pNDI-TVT-TET is a donor-acceptor (D-A) polymer that comprises electron rich unit (donor, TVT or TET) and electron poor unit (acceptor, NDI), differentiating it from aforementioned Thiophene-based polymers such as PEDOT and $\mathrm{p}\left(\mathrm{g}_{4} 2 \mathrm{~T}-\mathrm{T}\right)$. Similarly to other D-A polymers, pNDI-TVT-TET polymers have a small bandgap of $1.47 \sim 2.08 \mathrm{eV}$ with respect to the ratio of TVT to TET ${ }^{86}$ and exhibit ambipolar charge transport. Owing to the ambipolar charge transport, it is capable of conducting both electron and hole upon positive and negative voltage respectively. In a D-A polymer, the overlapping of $\mathrm{p}$ orbitals of donor and acceptor leads to a new smaller bandgap, as shown in Figure 10b. Moreover, it turned out that the ambipolar transport of pNDI-TVT-TET is strongly dependent on the ratio of TVT to TET. ${ }^{86}$

(a)

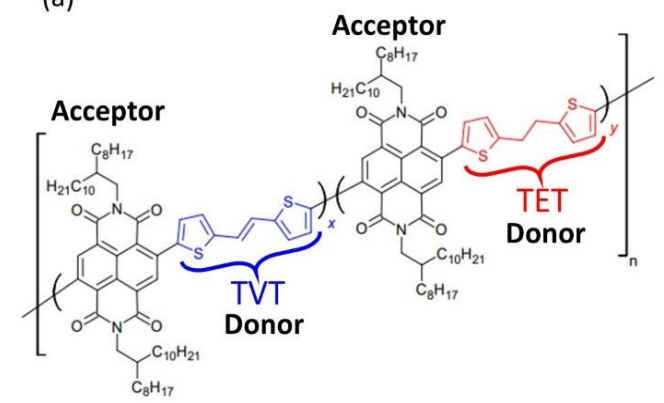

(b)

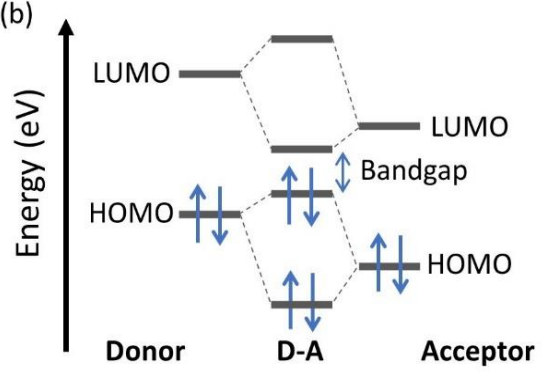

Figure 10 (a) Chemical Structure of pNDI-TVT-TET where NDI (black) is acceptor, and TVT (blue) and TET (red) are Donor, (b) Molecular orbital formed by overlap between Donor and Acceptor units in the Donor-Acceptor copolymer, resulting in a low bandgap

As described above, the key to engineering pNDI-TVT-TET is to vary the ratio of TVT to TET. Therefore, the backbone of polymer chains has a different conjugation length since TET is a $\pi$ - nonconjugated unit, while TVT is a $\pi$-conjugated one. As a result of changes in conjugation length, the morphology of films and the electronic structure are affected, leading to adjustment of the conductivity and mechanical properties. ${ }^{30,86}$ Both experimental and computational 
studies found that the glass transition temperature of the films increases as the TVT content increases. Moreover, the change in morphology has a subtle impact on the electron mobility because the extended network of $\pi-\pi$ stacking is well preserved upon incorporation of $\pi$ nonconjugated unit (TET). ${ }^{30,86}$ The mechanical properties as function of a ratio of TVT/TET as well as the performance of flexible organic field effect transistors with pNDI-TVT-TET layer as a semiconductor layer are detailed in Paper V. 


\section{Mechanism of In-Situ chemical polymerization of PEDOT:TOS}

Among various polymerization methods for PEDOT:TOS where the positive charges of PEDOT chains are balanced by TOS ${ }^{-}$counter anions, in-situ chemical polymerization (ICP) is cost-beneficial over other methods such as VPP, CVD and EP. Since in-situ chemical polymerization is a solution process, it is capable of mass-production with low cost. Besides, PEDOT:TOS films from ICP exhibits high conductivity up to $2000 \mathrm{Scm}^{-1}$, while the highest conductivity $^{35}\left(8797 \mathrm{Scm}^{-1}\right)$ has been obtained from VPP.

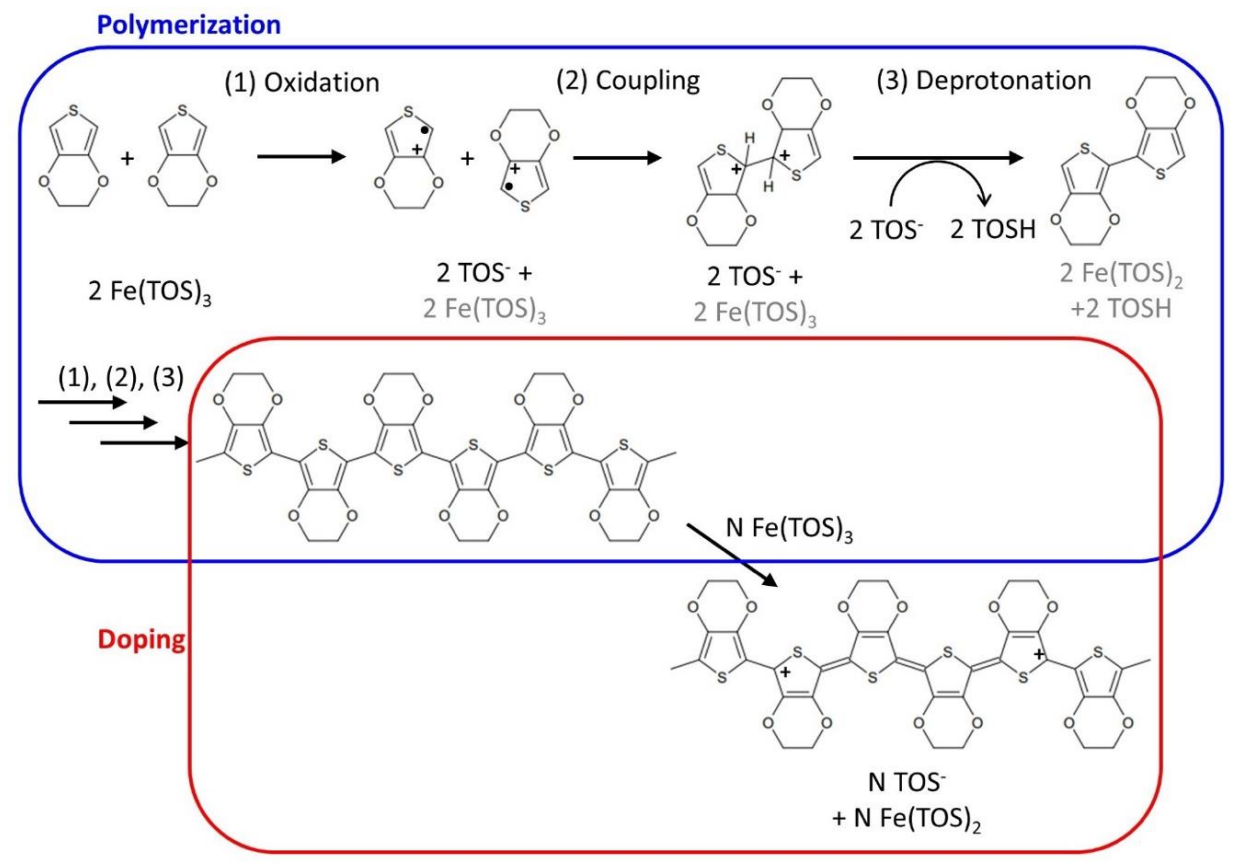

Figure 11 The mechanism of In-situ chemical polymerization of PEDOT with $\mathrm{Fe}(\mathrm{TOS})_{3}$ as oxidant (and dopant).

Experimental studies demonstrated the mechanism of in-situ chemical polymerization for PEDOT:TOS and determined its reaction rate. ${ }^{90,91}$ For the sake of clarification, in this thesis, we call it a conventional mechanism. The conventional mechanism consists of two parts: 1) Polymerization and 2) Doping, as described in Figure 11. In the polymerization, reactants (monomers (or oligomers) and oxidants) go through three steps: 1) oxidation, 2) Coupling 3) Deprotonation as elaborated below.

(1) Oxidation: Two neutral monomers are oxidized by oxidants, Iron Tosylate ( $\left.\mathrm{Fe}(\mathrm{TOS})_{3}\right)$, to two radical cations $\left(\mathrm{EDOT}^{\bullet+}\right)$ charges of which are balances by counter anions $\left(\mathrm{TOS}^{-}\right)$. 
(2) Coupling: Two radical cations form a bond to make an intermediate molecule that is a protonated dimer with two positive charges $\left(\mathrm{PEDOT}_{2}{ }^{2+}-2 \mathrm{H}\right)$ balanced by counter anions.

(3) Deprotonation: Finally, the charged intermediate molecule is deprotonated by TOS', resulting in a neutral dimer $\left(\mathrm{PEDOT}_{2}\right)$.

Repeating these three steps leads to long neutral PEDOT oligomers $\left(\mathrm{PEDOT}_{\mathrm{N}}\right)$ of length $\mathrm{N}$. After finishing polymerization, the neutral oligomers are doped by dopant $\left(\mathrm{Fe}(\mathrm{TOS})_{3}\right)$ that is a same as the oxidant for polymerization. Therefore, stoichiometrically, 2.33 moles equivalents of $\mathrm{Fe}(\mathrm{TOS})_{3}$ is required to produce PEDOT with a doping level of $33 \%$ where two moles equivalents are consumed to polymerize neutral oligomers, and 0.33 mole equivalents are used for doping the oligomers to $33 \%$ of oxidation level. As byproducts, Fe(TOS) $)_{2}$ and TOSH (pToluenesulfonic acid) are produced and afterwards removed by rinsing with for instance ethanol, butanol or/and water.

The reaction rate constant of each steps in the conventional mechanism was investigated. ${ }^{90}$ It turned out that the rate determining step of the polymerization is the oxidation of EDOT monomer to the radical cation $\left(\mathrm{k}_{1}=0.16 \mathrm{~L}^{3} \mathrm{~mol}^{-3} \mathrm{~h}^{-1}\right)$. Compared to this step, the rate of oxidation of dimer $\left(\mathrm{PEDOT}_{2}\right)$ is considerably fast $\left(\mathrm{k}_{1}{ }^{\prime}=3 \times 10^{3} \mathrm{Lmol}^{-1} \mathrm{~h}^{-1}\right)$. It is assumed that the rate of oxidation of longer oligomers than dimer is faster than or as fast as that of dimer. The rate of coupling is so fast $\left(\mathrm{k}_{2}=10^{9} \mathrm{Lmol}^{-1} \mathrm{~h}^{-1}\right)$ that it is considered to be constant for a coupling between long oligomer radical cations.

In addition to this mechanism of polymerization, another minor route is revealed in which added acids oxidize a neutral EDOT to a protonated EDOT with a positive charge. This protonated EDOT forms a bond with another EDOT. Afterwards, deprotonation of the intermediate molecule results in a neutral protonated dimer. The side reaction could proceed further to a formation of trimer. Even though the reaction rate of the side reaction is remarkably slow compared to the radical cation route (Figure 11), this additional route accelerates to some degree the rate of polymerization as defined by,

$$
r_{1}=k_{1} c_{E D O T}^{2} c_{\mathrm{Fe}(\mathrm{TOS})_{3}}^{2}+k_{11} c_{\mathrm{H}^{+}} \mathrm{C}_{\mathrm{EDOT}} \mathrm{C}_{\mathrm{Fe}(\mathrm{TOS})_{3}}
$$

Equation 3.1

where $r_{1}$ is the reaction rate, $k_{1}$ and $k_{11}$ are the rate constant for respectively the oxidation of EDOT and acceleration of proton protonating EDOT (the side reaction), and $c$ is the concentration. Further studies disclosed that the polymerization rate of ICP could be reduced by adding proton scavengers such as pyridine, imidazole, and NMP (N-methyl-2-pyrrolidone), and thereby the ICP results in PEDOT with higher molecular weight. ${ }^{92}$

In paper I, the conventional mechanism of ICP for PEDOT:TOS is verified by energy calculation via Density Functional Theory. In paper II, a polymerization model for ICP of PEDOT:TOS is introduced using molecular dynamics simulations in order to explore the effect of polymerization temperature on PEDOT length. 


\section{Mechanical Properties of Conducting polymer}

\subsection{Kinetic Fragility}

Under the glass transition temperature $\left(\mathrm{T}_{\mathrm{g}}\right)$, polymers are in glassy state. The glass is a disordered material that is non-crystalline structure and usually defined as a liquid without an ability to flow but with a mechanical behavior similar to solid. To utilize conducting polymers, the processability of conducting polymers is an indispensable feature since the fabrication process often involves the cooling process from liquid to glass state. The relaxation time (or viscosity) of polymers changes as temperature decreases, and thereby the crystallization would be disrupted during the allowed time by the cooling rate that is sufficiently fast.

Glass-forming materials could be classified by their temperature dependence of structural relaxation, especially near at their glass transition temperature $\left(T_{g}\right)$. At near $T_{g}$, the amorphous region of materials undergoes a transition from rubbery state to brittle glassy state with decreasing temperature or vice versa. This temperature-dependent behavior is referred to as '(kinetic) Fragility' that describes how rapidly the dynamics of glass-forming materials slows down upon (super) cooling.

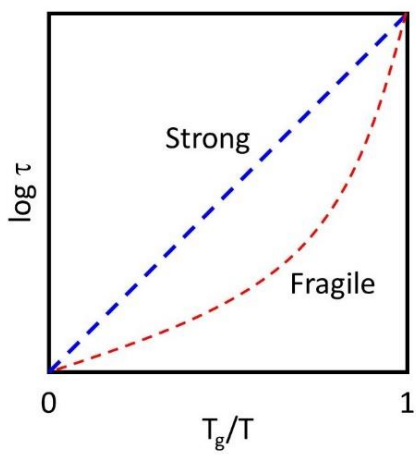

Figure 12 Conceptual scheme of Arrhenius plot of strong and fragile materials, (logarithmic) relaxation time vs $1 / T$ (normalized by $T_{g}$ ).

Angell et al proposed a useful classification model according to which there are 'strong' and 'fragile' glass formers (glass-forming materials). ${ }^{93}$ While the strong glass formers follow the Arrhenius law at near glass transition temperature upon cooling, the fragile glass formers show a considerable increase in relaxation time leading to deviation from Arrhenius law. Figure 12 represents a typical behavior of relaxation time with respect to inversed $\mathrm{T}_{\mathrm{g}}$-scaled temperature for strong and fragile materials. Please note that classification could be done as well by the correlation between the viscosity and temperature. Based on the classification model, the fragility of materials could be quantitatively evaluated by the fragility index defined as,

$$
m=\left.\frac{d \log _{10} \tau}{d\left(T_{g} / T\right)}\right|_{T=T_{g}}
$$

Equation 4.1.1 
where $\tau$ and $T$ are the structural relaxation time and the temperature respectively, while $T_{g}$ denotes the glass transition temperature. As the equation characterizes a deviation from the Arrhenius Law for the relaxation time, we can infer that the fragile materials have higher $\mathrm{m}$ value (i.e. steeper slope) than the strong materials due to their longer relaxation time. As most polymers are fragile, they have relatively high fragility index.

There have been a number of attempts to understand the fragility of polymers, ${ }^{94-98}$ but, it has not yet been fully understood. The fragility of polymers increases with an increase in the molecular weight, which is due to a less inefficient packing. ${ }^{94}$ In addition, polymers exhibit a strong $\mathrm{T}_{\mathrm{g}}$ dependence of fragility, whereas other glass-forming materials like inorganic glasses (e.g. silica) show $\mathrm{T}_{\mathrm{g}}$ independence of fragility. ${ }^{99}$ Besides, the fragility index of PMMA (Poly(methylmethacrylate)) increases as it is crosslinked by ethylene glycol dimethacrylate (EGDMA) ${ }^{100}$ The structural factors such as molecular weight, side chains, and crystallinity could affect the fragility of conducting polymers which could characterize the mechanical properties such as elastic modulus. ${ }^{96,98}$ 


\subsection{Elastic Modulus}

Engineering the mechanical properties of conducting polymers is essential to meet demands for stretchable (or flexible) organic electric devices. There are several ways to estimate the mechanical properties of polymers, and most of them measure a mechanical response (e.g. stress) of polymers to the applied load (e.g. strain). A typical way to obtain a stress-strain curve is through tensile strain test, as illustrated in Figure 13a where the tensile strain $\varepsilon$ is defined as,

$$
\varepsilon=\Delta \mathrm{L} / L_{0},\left(\Delta L=L-L_{0}\right)
$$

Equation 4.2.1

with $L$ and $L_{0}$ being the final and initial lengths of the sample, respectively. The stress-strain curve of tensile test reveals several mechanical properties of polymer such as the elastic modulus, yield point, and fracture point as illustrated in Figure 13b.

(a)

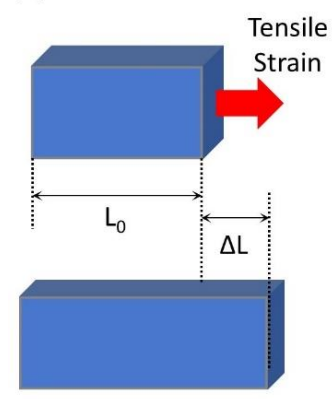

(b)

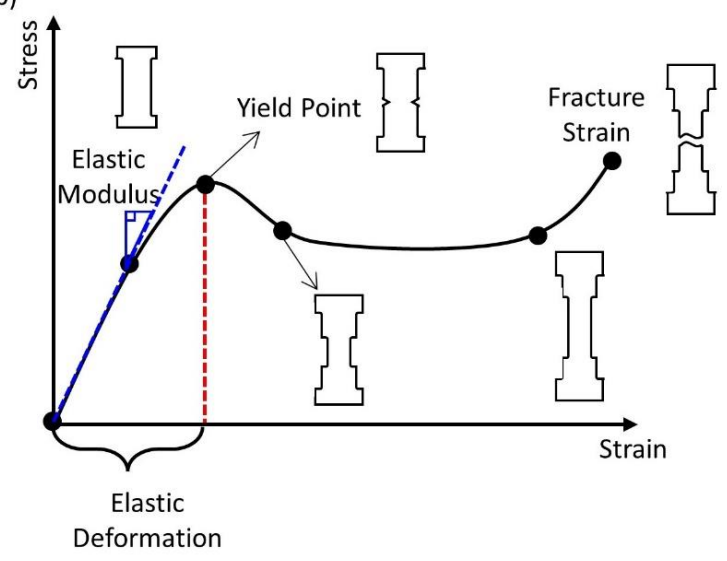

Figure 13 (a) conceptual scheme of tensile test (b) a typical example of stress-strain curve of the tensile test

In the stress-strain curve, there is a region with a linear response of stress with respect to the applied strain. This region is referred to as an elastic deformation where the slope corresponds to the elastic modulus (Young's modulus). The elastic modulus can be interpreted as a parameter that characterizes a capacity of mechanical energy that the polymer can store reversibly. After the linear region, the yield point emerges in the stress-strain curve corresponding to the point after which the sample deforms permanently, and the stress response becomes non-linear. The strain at which the sample eventually ruptures is referred as a fracture strain (or stress). The area under the entire curve indicates the toughness of the sample, while the area under linear region (Elastic Deformation) is the resilience.

Note that the stress-strain curve in Figure 13b corresponds to a typical ductile behaviour of polymer, whereas the curve of brittle polymers exhibits a different shape which shows high elastic modulus and no yield point. Since the backbones of conducting polymers are $\pi$ conjugated, they in general are stiffer than non-conjugated ones. This leads to several notable 
mechanical responses such as directional dependence of the elastic modulus that is attributed to the effect of $\pi-\pi$ stacking between chains. In spite of the importance of the mechanical properties of conducting polymer, it is challenging to investigate these mechanical properties since these properties are dependent on various factors such as blending, ${ }^{55,101}$ copolymerization, ${ }^{80}$ stain rate, ${ }^{102}$ molecular weight, ${ }^{102}$ side chain, ${ }^{103,104}$ and crystallinity. ${ }^{105}$

In addition, the films for electric devices made from conducting polymer are generally very thin $(<100 \mathrm{~nm})$, leading to a practical trouble when pulling conducting polymers that are not free-standing. To get around this problem in measurement, several measurement techniques are devised. One of these techniques is film-on-water where a thin film is placed on the surface of water and uniaxial tensile strain is applied. Another approach is to measure elastic modulus by means of Atomic Force Microscopy. ${ }^{106}$ It should be noted that the technique with AFM does not apply tensile strain, while it measures the force responding to compression by AFM tip (probe). Although there are several useful experimental approaches for elastic modulus measurement, each technique is still greatly influenced by the condition of measurement, for example interaction with water in film-on-water technique or the shape of tip in AFM technique. Therefore, computational approaches can be a strong tool to investigate the mechanical properties since it is facile to investigate the morphology of films on molecular level and analyze the interaction energy during deformation test. 


\section{Theory and Computational Methods}

For last several decades, the role of computational methods has appeared indispensable in the research of materials, which is driven by exponentially increased computational power and development of advanced computational methods, for instance the density functional theory and molecular dynamics. The research by computational methods has not only provided theoretical understandings on materials that experimental studies can rely on, but also reduced the number of experiments based on the trials-and-errors approach. In this chapter, the fundamental knowledge of computational methods utilized in the thesis is introduced.

\subsection{First principal Calculation}

\subsubsection{Schrödinger Equation and Many-body problem}

A quantum mechanical system composed of particles (electrons and nuclei) is described by a wave function $(\Psi),{ }^{107}$ whose squared modulus $\left(|\Psi|^{2}\right)$ corresponds to the probability density to find a particle at a given position and time. The evolution of the wave function is governed by the time-dependent Schrödinger equation

$$
i \frac{\partial \Psi(\vec{r}, t)}{\partial t}=\widehat{H} \Psi(\vec{r}, t)
$$

Equation 5.1.1.1

where $\widehat{H}$ denotes the Hamiltonian operator. The wave function could be expressed by a product of spatial and temporal terms as defined,

$$
\Psi(\vec{r}, t)=\Psi(\vec{r}) f(t)
$$

Equation 5.1.1.2

The time-independent Schrödinger equation could be derived from the time-dependent Schrödinger equation as expressed in Equation 5.1.1.3 given that the Hamiltonian Operator is independent of time.

$$
\widehat{H} \Psi(\vec{r})=E \Psi(\vec{r})
$$

Equation 5.1.1.3

In the Schrödinger equation, $E$ corresponds to the energy of the system. In case of a system composed of $\mathrm{M}$ nuclei and $\mathrm{N}$ electrons, the Hamiltonian operator of the time-independent Schrödinger equation could be expressed as below,

$$
\begin{gathered}
\widehat{H}=-\frac{1}{2} \sum_{A=1}^{M_{\text {nuclei }}} \frac{1}{M_{A}} \nabla_{A}^{2}-\frac{1}{2} \sum_{i=1}^{N_{\text {elec }}} \nabla_{i}^{2}+\sum_{A=1}^{M_{\text {nuclei }}} \sum_{B>A}^{M_{\text {nuclei }}} \frac{Z_{A} Z_{B}}{\vec{R}_{A}-\vec{R}_{B}} \quad \text { Equation 5.1.1.4 } \\
+\sum_{i=1}^{N_{\text {elec }}} \sum_{j>i}^{N_{\text {elec }}} \frac{1}{\vec{r}_{i}-\vec{r}_{j}}-\sum_{i=1}^{N_{\text {elec }}} \sum_{A=1}^{M_{\text {nuclei }}} \frac{Z_{A}}{\vec{r}_{i}-\vec{R}_{A}} \\
=\widehat{T}_{n}+\widehat{T}_{e}+\widehat{V}_{n n}+\widehat{V}_{e e}+\widehat{V}_{\text {en }}
\end{gathered}
$$


where $M_{A}, \vec{R}_{A}$ (or $\vec{R}_{B}$ ), and $Z_{A}$ (or $Z_{B}$ ) denotes the mass, coordinate and charge of $A^{\text {th }}$ (or $B^{\text {th }}$ ) nucleus respectively and $\vec{r}_{i}$ (or $\vec{r}_{j}$ ) is the coordinate of $i^{\text {th }}$ (or $j^{\text {th }}$ ) electron. The first two terms are the kinetic energy for nucleus $\left(\widehat{T}_{n}\right)$ and electron $\left(\widehat{T}_{e}\right)$. The third, fourth, and fifth term in the equation correspond to nucleus-nucleus $\left(\widehat{V}_{n n}\right)$, electron-electron $\left(\widehat{V}_{e e}\right)$, and electron-nucleus $\left(\widehat{V}_{e n}\right)$ interaction, respectively. $\nabla_{A}^{2}$ and $\nabla_{i}^{2}$ are the Laplace operator (or Laplacian) for nucleus $\left(\vec{R}_{A}=\left(X_{A}, Y_{A}, Z_{A}\right)\right)$ and electron $\left(\vec{r}_{i}=\left(x_{i}, y_{i}, z_{i}\right)\right)$ defined as,

$$
\begin{aligned}
& \nabla_{A}^{2}=\frac{\partial^{2}}{\partial X_{A}^{2}}+\frac{\partial^{2}}{\partial Y_{A}^{2}}+\frac{\partial^{2}}{\partial Z_{A}^{2}} \\
& \nabla_{i}^{2}=\frac{\partial^{2}}{\partial x_{i}^{2}}+\frac{\partial^{2}}{\partial y_{i}^{2}}+\frac{\partial^{2}}{\partial z_{i}^{2}}
\end{aligned}
$$

Equation 5.1.1.5

It should be noted that the equation is in atomic units where the mass of electron, modulus of charge of electron, and reduced Planck's constant are unity. Since solving the many-body Schrödinger equation is practically impossible, a variety of approximations have been suggested as described below.

According to the Born-Oppenheimer (OP) approximation, as the nuclei are substantially heavier than the electrons, the nuclei are considered as fixed. Moreover, the total wave function of a molecule could be represented as the product of the electronic and nuclear wave function $\left(\Psi_{\text {total }}=\Psi_{\text {elec }} \Psi_{\text {nucl }}\right)$. As a result, the electronics Hamiltonian operator can be re-written without the kinetic energy of nuclei $\left(\widehat{T}_{n}\right)$ in Equation 5.1.1.4 as below,

$$
\widehat{H}_{e}=\widehat{T}_{e}+\widehat{V}_{n n}+\widehat{V}_{e e}+\widehat{V}_{e n}
$$

Equation 5.1.1.6

In Equation 5.1.1.6, the $1^{\text {st }}\left(\widehat{T}_{n}\right)$ and $3^{\text {rd }}\left(\widehat{V}_{n n}\right)$ terms of Equation 5.1.1.4 are treated as follows: the kinetic energy of nuclei $\left(\widehat{T}_{n}\right)$ is omitted and the nucleus-nucleus interaction $\left(\widehat{V}_{n n}\right)$ is constant. Solving the Schrödinger Equation with the electronic Hamiltonian gives the electronic wave function $\left(\Psi_{\text {elec }}\right)$ and electronic energy $\left(E_{\text {elec }}\right)$. Although the Born-Oppenheimer approximation reduces the complexity of solving the Schrödinger Equation, it is still impossible to solve the equation exactly for systems with more than one electron (e.g., hydrogen atom $(\mathrm{H})$ and $\mathrm{H}_{2}{ }^{+}$). In the following sections, a various of approximations such as Hartree-Fock and Density Functional Theory are described to solve many-body problems.

\subsubsection{Hartree-Fock method}

In Equation 5.1.1.6, the electronic Hamiltonian depends exclusively on the coordinate of electrons. In addition, electron spin should be included to the (molecular or atomic) orbital. It is possible to take the electron spin-dependent effect into consideration in an ad-hoc fashion in non-relativistic theory. To this end, an extra spin coordinate is added to spatial coordinate $\left(\vec{r}_{i}\right)$ as below.

$$
\vec{x}_{i} \equiv\left\{\vec{r}_{i}, \omega_{i}\right\}
$$

Equation 5.1.2.1

where $\vec{x}_{i}, \vec{r}_{i}$, and $\omega_{i}$ are the space-spin coordinate, spatial coordinate, and spin coordinate of electrons. The corresponding spin functions are denoted by $\alpha$ and $\beta$, also known as up- and 
down spin. The spin-dependent effect of a molecular orbital $(\Psi(\vec{x}))$, also known as spin orbital, could be introduced by multiplying a spin function to a spatial orbital as defined,

$$
\psi(\vec{x})=\left\{\begin{array}{l}
\phi^{\alpha}(\vec{r}) \alpha(\omega) \\
\phi^{\beta}(\vec{r}) \beta(\omega)
\end{array}\right.
$$

Equation 5.1.2.2

where $\phi^{\alpha}$ (or $\phi^{\beta}$ ) is the spatial orbital and $\alpha(\omega)$ (or $\beta(\omega)$ ) is the up-spin (or down-spin) function. The spin orbitals satisfy the orthonormality condition.

$$
\left\langle\psi_{i} \mid \Psi_{j}\right\rangle \equiv \int \Psi_{i}{ }^{*} \Psi_{j} d \vec{x}_{i} d \vec{x}_{j}=\delta_{i j} \equiv\left\{\begin{array}{l}
1, \text { when } i=j \\
0, \text { otherwise }
\end{array}\right.
$$

Equation 5.1.2.3

The total wave function for a system with $\mathrm{N}$ electrons should be antisymmetric, resulting in the Pauli principle according to which two electrons cannot have all quantum numbers equal. The anti-symmetry requirement means that the sign of wave function changes with an interchange of any two electron coordinates $\left(\vec{x}_{i}\right.$ and $\left.\vec{x}_{j},(j \neq i)\right)$. The antisymmetric wave function can be built by Slater determinants as given in Equation 5.1.2.4.

$$
\Psi\left(\vec{x}_{1}, \vec{x}_{2}, \vec{x}_{3}, \ldots, \vec{x}_{N}\right)=\frac{1}{\sqrt{N !}}\left|\begin{array}{ccc}
\Psi_{1}\left(\vec{x}_{1}\right) & \cdots & \Psi_{N}\left(\vec{x}_{1}\right) \\
\vdots & \ddots & \vdots \\
\psi_{1}\left(\vec{x}_{N}\right) & \cdots & \Psi_{N}\left(\vec{x}_{N}\right)
\end{array}\right|
$$

Equation 5.1.2.4

With a normalized wave function, the energy of system can be expressed as in Equation 5.1.2.5.

$$
E_{\text {elec }}=\left\langle\Psi\left|\widehat{H}_{e}\right| \Psi\right\rangle \equiv \int \Psi^{*} \widehat{H}_{e} \Psi d \vec{x}_{1} d \vec{x}_{2} \cdots d \vec{x}_{N}
$$

Equation 5.1.2.5

The variational theorem which states that the energy is always greater than the true energy can be employed to minimize the energy. A better approximate wavefunction that gives a lower $E_{\text {elec }}$ can be obtained by employing the variational theorem. Therefore, the best approximate wave function that gives the minimum energy can be obtained by iterative minimization of energy. In Hartree-Fock (HF) approximation, a single Slater determinant is used for the trial wave function, corresponding to an assumption that each electron moves independently to all other electrons, but the electron-electron interaction (repulsion) is considered as an average effect due to the average positions of all electrons. Therefore, the electronic Hamiltonian could be further simplified as a sum of one-electron operators.

First, one-electron operator is defined as

$$
\hat{h}=-\frac{1}{2} \nabla_{i}^{2}-\sum_{A=1}^{M_{\text {nuclei }}} \frac{Z_{A}}{\vec{r}_{l}-\vec{R}_{A}}
$$

Equation 5.1.2.6

A two-electron operator is given in Equation 5.1.2.7.

$$
\hat{v}_{i j}=\frac{1}{\vec{r}_{i}-\vec{r}_{j}}
$$

Hence, the electronic Hamiltonian for a system with $\mathrm{M}$ nuclei and $\mathrm{N}$ electrons can be written simply as,

$$
\widehat{H}_{e}=\sum_{i=1}^{N_{\text {elec }}} \hat{h}_{i}+\sum_{j>i}^{N_{\text {elec }}} \hat{v}_{i j}+\widehat{V}_{n n}
$$


where the nucleus-nucleus repulsion term $\widehat{V}_{n n}$ is constant for a given nuclear coordinates and independent to electron coordinates. Therefore, for the sake of convenience, $\widehat{V}_{n n}$ term is neglected in the following section since it does not affect the eigenfunctions but changes the eigenvalues.

With the simplified Hamiltonian by HF approximation the energy of the system could be written as, ${ }^{107,108}$

$$
E_{\text {elec }}=\sum_{i=1}^{N_{\text {elec }}} h_{i}+\frac{1}{2} \sum_{i=1}^{N_{\text {elec }}} \sum_{j=1}^{N_{\text {elec }}}\left(J_{i j}-K_{i j}\right)
$$

Equation 5.1.2.9

where,

$$
\begin{aligned}
h_{i}= & \left\langle\psi_{i}\left|\hat{h}_{i}\right| \psi_{i}\right\rangle=\int d \vec{x}_{1} \psi_{i}^{*}\left(\vec{x}_{1}\right) \hat{h}_{i} \psi_{i}\left(\vec{x}_{1}\right) \\
& J_{i j}=\left\langle\Psi_{i} \Psi_{j}\left|\hat{v}_{i j}\right| \psi_{i} \psi_{j}\right\rangle \equiv\langle i j \mid i j\rangle \\
= & \int d \vec{x}_{1} d \vec{x}_{2} \psi_{i}^{*}\left(\vec{x}_{1}\right) \psi_{j}^{*}\left(\vec{x}_{2}\right) \hat{v}_{i j} \psi_{i}\left(\vec{x}_{1}\right) \psi_{j}\left(\vec{x}_{2}\right) \\
& K_{i j}=\left\langle\psi_{i} \psi_{j}\left|\hat{v}_{i j}\right| \psi_{j} \Psi_{i}\right\rangle \equiv\langle i j \mid j i\rangle \\
= & \int d \vec{x}_{1} d \vec{x}_{2} \psi_{i}^{*}\left(\vec{x}_{1}\right) \psi_{j}^{*}\left(\vec{x}_{2}\right) \hat{v}_{i j} \psi_{j}\left(\vec{x}_{1}\right) \psi_{i}\left(\vec{x}_{2}\right)
\end{aligned}
$$

In Equation 5.1.2.9, $h_{i}$ is the one-electron integral for the kinetic energy of electron and attractive interaction between electron and nuclei, and $J_{i j}$, and $K_{i j}$ are the Coulombic integral and Exchange integral between electrons, respectively. It should be noted that when $i=j, J_{i i}$ is canceled by $K_{i i}$.

By determining a set of molecular orbitals (MO) which minimizes the energy based on the variational theorem under a condition that MOs remain ortho-normalized, the HK equation could be derived as follows,

$$
\hat{F} \psi_{i}(\vec{x})=\varepsilon_{i} \psi_{i}(\vec{x})
$$

where $\hat{F}$ is the Fock operator that consists of one-electron operator $(\boldsymbol{h})$, Coulomb $(\boldsymbol{J})$ and exchange $(\boldsymbol{K})$ operators as expressed in following three equations.

$$
\begin{aligned}
\hat{F}= & -\frac{1}{2} \nabla^{2}-\sum_{A}^{M_{\text {nuclei }}} \frac{Z_{A}}{\overrightarrow{\vec{r}}-\vec{R}_{A}}+\int \frac{\rho\left(\vec{x}^{\prime}\right)}{\vec{r}_{1}-\vec{r}_{2}} d \vec{x}^{\prime} \\
& -\int \frac{\rho\left(\vec{x}, \vec{x}^{\prime}\right) \psi_{i}\left(\vec{x}^{\prime}\right)}{\vec{r}_{1}-\vec{r}_{2}} d \vec{x}^{\prime}
\end{aligned}
$$

In

Equation 5.1.2.14, $\rho\left(\vec{x}^{\prime}\right)$ and $\rho\left(\vec{x}, \vec{x}^{\prime}\right)$ are electron density as defined,

$$
\rho(\vec{x})=\sum_{i} \psi_{i}^{*}(\vec{x}) \psi_{i}(\vec{x})=\left|\psi_{i}(\vec{x})\right|^{2}
$$




$$
\rho\left(\vec{x}, \vec{x}^{\prime}\right)=\sum_{i} \psi_{i}^{*}(\vec{x}) \psi_{i}\left(\vec{x}^{\prime}\right)
$$

A molecular orbital (MO) could be constructed by a linear combination of atomic orbitals (LCAO) as given in Equation 5.1.2.17.

$$
\psi_{i}(\vec{x})=\sum_{\mu=1}^{K_{\text {basis }}} C_{\mu i} g_{\mu}(\vec{x})
$$

Equation 5.1.2.17

where $C_{\mu i}$ and $g_{\mu}(\vec{x})$ the coefficient of LCAO and a basis set, respectively. The use of MO from LCAO could transform the HF equation (Equation 5.1.2.13) into the Hartree-FockRoothaan equations as defined,

$$
\begin{gathered}
\boldsymbol{F C}=\boldsymbol{S C} \varepsilon \\
F_{\mu \nu}=\left\langle g_{\mu}|\hat{F}| g_{\nu}\right\rangle \\
S_{\mu \nu}=\left\langle g_{\mu} \mid g_{\nu}\right\rangle
\end{gathered}
$$

Equation 5.1.2.18

Equation 5.1.2.19

Equation 5.1.2.20

In Equation 5.1.2.18, $\boldsymbol{F}$ is the Fock matrix in which each element $F_{\mu \nu}$ involves integrals with Fock operator as given in Equation 5.1.2.19, resulting in one-electron integrals and twoelectron integrals multiplied by a density matrix that is a sum over occupied MOs of coefficients. The overlap matrix $S$ in Equation 5.1.2.20 contains the overlap elements between basis sets. And $\varepsilon$ denotes a diagonal matrix of the orbital energy $\varepsilon_{i}$. With given MO coefficients, one can determine the eigenvalue of Fock matrix in HF-Roothaan equation. However, the Fock matrix needs be diagonalized to obtain the MO coefficients. Therefore, the equation should be solved iteratively with some initial guess of the coefficients. This is why solving Hartree-Fock equation is referred to as a self-consistent field (SCF) procedure.

\subsubsection{Density Functional Theory}

Although HF method enables solving a many-body system, it still allows the considerable number of degrees of freedom of system since the wavefunction (Molecular Orbital) is used to solve HF. In 1964, Density Functional Theory (DFT) based on Hohenberg-Kohn theorems was introduced. $^{107,109}$

Theorem I: For any electronic system, the external potential $\left(v_{\text {ext }}(\vec{r})\right)$ is determined by the ground state density $\rho_{0}(\vec{r})$.

Theorem II: A universal functional of the energy $E[\rho]$ can be defined in terms of the density that is valid for any external potential. For any particular $v_{\text {ext }}(\vec{r})$, the exact ground state of the system is determined from global minimum value of the functional.

In other words, these theorems state that the ground state electron density $\rho_{0}$ can be obtained by minimizing the energy functional of the electron density by applying the variational theorem. The energy functional of electron density is defined as follows,

$$
E[\rho]=T[\rho]+E_{n e}[\rho]+J[\rho]+E_{x c}[\rho]
$$

Equation 5.1.3.1 


$$
E_{n e}[\rho]=\int d \vec{r} \rho(r) v_{\text {ext }}(\vec{r})
$$

In Equation 5.1.3.1, the first term is the kinetic energy of electrons, the second term is nucleielectron attraction, the third term is the classical coulomb interaction between electrons (repulsion), and the fourth term is the non-classical self-interaction correction that will be further addressed below. In addition, while the second term (nuclei-electron potential energy) is dependent to the system, the other terms are not, indicating that they are universal functionals. In the universal part, even though the classical coulomb interaction $(J[\rho])$ is known, some trial functionals of the kinetic energy $(T[\rho])$ and non-classical term $\left(E_{x c}[\rho]\right)$ such as Thomas-FermiDirac model have exhibited too low accuracy for a practical use. ${ }^{107}$

Kohn and Sham proposed that it is possible to replace an interacting many-electron systems with a system of non-interacting electrons that move in an effective potential. ${ }^{110}$ In this approach, the ground state electron density of the interacting system is identical to that of noninteracting system. Moreover, orbitals are introduced again in DFT by employing a single slater determinant as in Hartree-Fock method, increasing the complexity significantly (e.g. for $\mathrm{N}$ particle system: $3 \rightarrow 3 N$ ). Applying the Kohn-Sham (KS) theory, the energy functional (Equation 5.1.3.1), particularly the universal part, can be reformulated to

$$
E[\rho]=T_{S}[\rho]+E_{n e}[\rho]+J[\rho]+E_{x c}[\rho]
$$

Equation 5.1.3.3

where $T_{S}[\rho]$ is the non-interacting kinetic energy. The last term $\left(E_{x c}[\rho]\right)$ in Equation 5.1.3.3 is the exchange-correlation energy that is a sum of remains after subtraction of non-interacting kinetic energy and classical coulomb interaction from the exact kinetic energy and exact coulomb interaction potential, respectively, resulting in the following equation,

$$
E_{x c}[\rho]=\left(T[\rho]-T_{s}[\rho]\right)+\left(E_{e e}[\rho]-J[\rho]\right)
$$

Equation 5.1.3.4

where the first and second parentheses corresponds the kinetic correlation energy and the exchange-correlation energy, respectively. In general, the kinetic correlation energy is smaller than the exchange-correlation energy, and therefore $E_{x c}[\rho]$ is usually called as the exchangecorrelation energy. From Equation 5.1.3.3, the Kohn-Sham equation could be defined as

$$
\left(-\frac{1}{2} \nabla_{i}^{2}+v_{e f f}(\vec{r})\right) \phi_{i}(\vec{r})=\varepsilon_{i} \phi_{i}(\vec{r})
$$

Equation 5.1.3.5

here, $\phi_{i}$ is the Kohn-Sham orbital for the single electron. The electron density with the KohnSham orbitals can be determined as

$$
\rho(\vec{r})=\sum_{i}^{N_{\text {elec }}}\left|\phi_{i}(\vec{r})\right|^{2}
$$

Equation 5.1.3.6

In Equation 5.1.3.5, $v_{\text {eff }}(\vec{r})$ is the effective potential defined as,

$$
v_{e f f}(\vec{r})=v_{n e}(\vec{r})+\int \frac{\rho\left(\vec{r}^{\prime}\right)}{\vec{r}-\vec{r}^{\prime}} d \vec{r}^{\prime}+v_{x c}(\vec{r})
$$

where $v_{x c}(\vec{r})$ is exchange-correlation potential and it could be obtained from the derivative of the exchange-correlation energy $\left(E_{x c}[\rho]\right)$. 


$$
v_{x c}(\vec{r})=\frac{\delta E_{x c}[\rho]}{\delta \rho}
$$

The Kohn-Sham equation can be solved by SCF method similarly to the Hartree-Fock equation as illustrated in Figure 14.

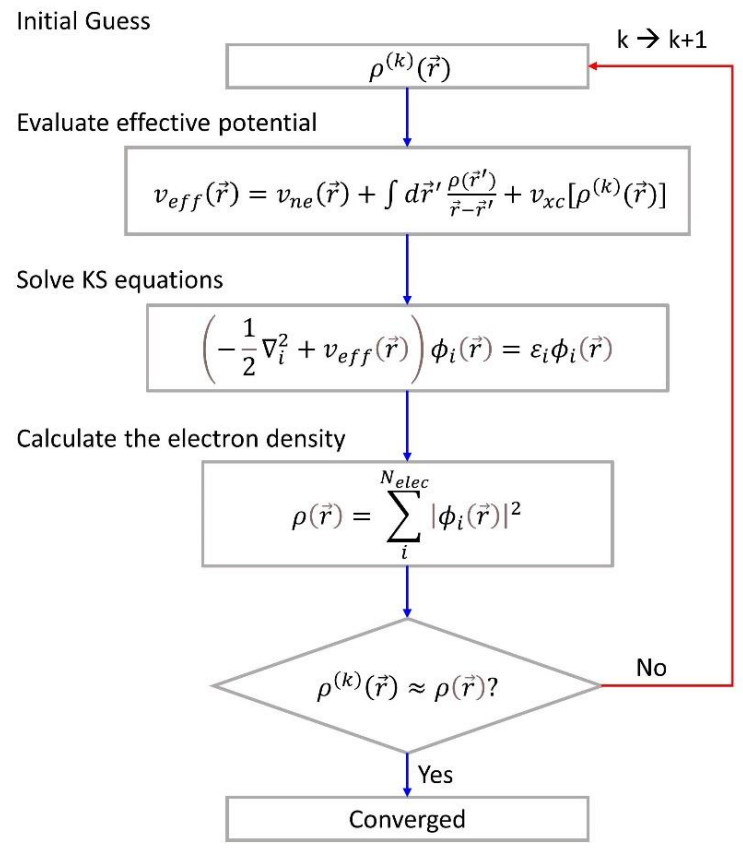

Figure 14 The self-consistency loop in DFT 


\subsubsection{Exchange-correlation Functionals}

Since the exchange-correlation energy functional can only be approximated, it is the main challenge to find a "good enough" functional for $E_{x c}[\rho]$. Among several attempts at developing approximations, the Local Density Approximation (LDA) is the most common functional. In LDA, the $E_{x c}[\rho]$ is assumed to be a local function of the electron density that is treated as a locally uniform electron gas, leading to,

$$
E_{x c}^{L D A}[\rho]=\int \rho(\vec{r}) \varepsilon_{x c}(\rho(\vec{r})) d \vec{r}
$$

Equation 5.1.4.1

Here, $\varepsilon_{x c}(\rho(\vec{r}))$ is the exchange-correlation energy for the homogeneous electron gas and is weighted by the electron density $(\rho(\vec{r}))$. It is noteworthy that the exchange-correlation energy term, $\varepsilon_{x c}(\rho(\vec{r}))$, can be expressed in a sum of exchange and correlation contribution $\left(\varepsilon_{x c}(\rho(\vec{r}))=\varepsilon_{x}(\rho(\vec{r}))+\varepsilon_{c}(\rho(\vec{r}))\right)$. The LDA describes homogeneous systems very well such as free-electron system (e.g. metals), whereas it shows deficient accuracy for weakly bonded systems (e.g. a hydrogen molecule binding on metal surface). ${ }^{11}$ Another popular approximation is the Generalized Gradient Approximation (GGA). In the GGA, the gradient of the charge density is taken into account as defined,

$$
E_{x c}^{G G A}[\rho]=\int f[\rho(\vec{r}), \nabla \rho(\vec{r})] d \vec{r}
$$

Equation 5.1.4.2

A various of hybrid functionals that corporate the Hartree-Fock exchange functional $E_{X}^{H F}$ into density functional approximations such as LDA (or LSDA: Local Spin Density Approximation) or GGA have been developed to improve the accuracy of calculations such as Ionization energy and bandgap. B3LYP is a hybrid functional ${ }^{112}$ as defined,

$$
\begin{aligned}
E_{x C}^{B 3 L Y P}= & (1-A) E_{X}^{L S D A}+A E_{X}^{H F}+B \Delta E_{X}^{B 88} \\
& +C E_{C}^{L Y P}+(1-C) E_{C}^{V W N}
\end{aligned}
$$

Equation 5.1.4.3

In Equation 5.1.4.3, $E_{X}^{L S D A}$ is the Slater exchange functional ${ }^{113}$ (local density exchange), $E_{X}^{H F}$ is Hartree-Fock exchange term, $\Delta E_{X}^{B 88}$ represents Becke's gradient correction to the exchange functional, ${ }^{114}$ and the last two terms $\left(E_{C}^{L Y P}\right.$ and $\left.E_{C}^{V W N}\right)$ are $L Y P^{115}$ and $\mathrm{VWN}^{116}$ correlation functionals, respectively. A, B, and $\mathrm{C}$ in Equation 5.1.4.3 are the empirical parameters obtained from fitting to experimental data, while a typical set of them are $\mathrm{A}=0.2, \mathrm{~B}=0.72$ and $\mathrm{C}=$ 0.81 . B3LYP tends to underestimate the long-range exchange contribution since while longterm exchange primarily arises from Hartree-Fock exchange, B3LYP includes only about 20\% of it.

To take into account long range interaction, a long-range corrected hybrid density functional $\omega \mathrm{B} 97 \mathrm{XD}^{117}$ has been developed by adding an empirical atom-atom dispersion correction $\left(E_{D}\right)$ to $\omega \mathrm{B} 97 \mathrm{X}$ functional ${ }^{118}$ as given in Equation 5.1.4.4.

$$
E_{x c}^{\omega b 97 X D}=E_{x}^{L R-H F}+c_{x} E_{x}^{S R-H F}+E_{x}^{S R-B 97}+E_{c}^{B 97}+E_{D} \quad \text { Equation 5.1.4.4 }
$$

In Equation 5.1.4.4, the first four terms are $\omega \mathrm{B} 97 \mathrm{X}$ functional $\left(E_{x c}^{\omega b 97 X}\right)$ where $E_{x}^{L R-H F}$ and $E_{x}^{S R-H F}$ are long-range and short-range $\mathrm{HF}$ exchange functionals, $c_{x}$ is a fractional number for $E_{x}^{S R-H F}, E_{x}^{S R-B 97}$ is the short-range B97 exchange functional, and $E_{c}^{B 97}$ is the B97 correlation functional. The dispersion correction $\left(E_{D}\right)$ is defined as, 


$$
E_{D}=-\sum_{i=1}^{N_{\text {atom }}-1} \sum_{j=a+1}^{N_{\text {atom }}} \frac{C_{6}^{i j}}{R_{i j}^{6}} f_{D}\left(R_{i j}\right)
$$

where, $N_{\text {atom }}$ is the number of atoms, $C_{6}^{i j}$ and $R_{i j}$ denote the dispersion coefficient and distance of an atom-pair $i$ and $j$, and $f_{D}\left(R_{i j}\right)$ is a damping function introduced to correct zero dispersion at short interatomic distance and asymptotic pairwise Van der Waals (VdW) potential. The introduction of the dispersion correction improves the accuracy for not only non-covalent and covalent interactions as well as the thermochemical calculation and orbital energy. The $\omega \mathrm{B} 97 \mathrm{XD}$ is employed in DFT calculations of all papers included to this thesis.

\subsubsection{Basis Sets}

To solve the many-body Schrödinger equation, it is necessary to introduce a basis set to construct molecular orbitals. As presented in Equation 5.1.2.17, the linear combination of atomic orbitals (a basis set) is generally employed to construct the molecular orbitals (MO). Since it is impossible in an actual calculation to use a complete basis set composed of infinite basis functions, it is practical to use a finite basis set to build MOs. Therefore, the accuracy of calculation is dependent to the number of functions in the basis set. Therefore, the number of basis functions is crucial to how well the MOs are represented. In general the smaller the basis set, the poorer representation of MOs.

For the basis functions, Slater type orbitals (STOs) and Gaussian type orbitals (GTOs) are widely used to model the atomic orbitals of molecules as defined below,

$$
\begin{gathered}
\chi_{\zeta, n, l, m}^{S T O}(r, \theta, \varphi)=N Y_{l, m}(\theta, \varphi) r^{n-1} e^{-\zeta r} \\
\chi_{\zeta, n, l, m}^{G T O}(r, \theta, \varphi)=N Y_{l, m}(\theta, \varphi) r^{2 n-2-l} e^{-\zeta r^{2}}
\end{gathered}
$$

Equation 5.1.5.1

Equation 5.1.5.2

Here, $\mathrm{N}$ is the normalization constant, $n, l$ and $m$ are quantum numbers, $Y_{l, m}$ is the spherical harmonic function, $\zeta$ is the constant related to the charge of the nucleus, and $r$ is the distance between the electron and the nucleus. Apart from STOs and GTOs, plane wave basis functions are widely used for periodic systems such as periodic crystals (i.e. a unit cell with periodic boundary conditions), and the number of basis functions for a plane wave basis set is generally larger than a basis set composed of atomic orbitals (STOs or GTOs). The STOs and GTOs will be further explained in this section since molecules that are not periodic are of interest in the thesis.

Although the integration of GTOs is computationally more efficient than integration of STOs, they have difficulties to represent the orbitals accurately near and far away from nucleus because of their $r^{2}$ dependence in the exponent. Therefore, more GTOs, usually three times as many GTOs as STOs, are required to achieve a certain accuracy. As a practical solution of the above challenges of STOs and GTOs, contracted Gaussian type orbital (CGTO) was introduced. CGTO is a linear combination of the primitive GTOs to mimic (the behavior of) STOs. A CGTO with a notation of STO $-n \mathrm{G}$ basis set corresponds to a linear combination of $n$ primitive GTOs to model the STO. 
The accuracy of basis sets could be improved by introduction of split-valence basis sets, polarization function, or diffuse function. The split-valence basis set is to employ multiple basis functions for one valence orbital. The simplest case is to double the number of all basis functions (i.e., two basis functions for one orbital), called as Double Zeta (DZ). In the case of three and four basis functions for one valence orbital, it is Triple-Zeta (TZ) and QuadrupleZeta (QZ), respectively. The use of the split-valence basis like DZ and TZ, allows to represent better the distribution of electrons in a molecule where the distribution is influenced by other nuclei and electrons as well as the type of bond (e.g., $\sigma$ - or $\pi$ - bonds). Polarization functions could be included to basis sets by using higher angular momentum function, i.e., p-orbitals added to s orbital, d-orbital to p-orbital, and f-orbital to d-orbital. The diffuse functions that are GTOs with smaller $\zeta$ could be added to the basis sets to give a better description of relatively diffuse electron distribution (e.g., anions).

Pople-type basis sets are a split-valence type with a notation of $A-Z_{1} Z_{2} Z_{3} \mathrm{~g}(\mathrm{X})$. This notation provides information about the number of primitive functions for core orbitals $(A-)$ and valence orbitals $\left(Z_{1} Z_{2} Z_{3}\right)$ as well as about the presence of polarization functions $(\mathrm{X})$ and diffuse functions. ' $A$ ' before the dash denotes the number of primitive GTOs for the core orbitals, and $Z_{1} Z_{2}\left(Z_{3}\right)$ denotes the type of the split-valence, i.e., $Z_{1} Z_{2}$ for $\mathrm{DZ}$ type and $Z_{1} Z_{2} Z_{3}$ for $\mathrm{TZ}$ where $Z_{1}, Z_{2}$ and $Z_{3}$ corresponds to the number of primitive GTOs for each basis function. The presence of the diffuse functions are expressed by ' $+(+)$ ' before $\mathrm{g}(\mathrm{X})$ in which $\mathrm{X}$ denotes the type of the polarization functions that are added, e.g. d, p, or f. For example, 6-31g(d) is a polarized basis set where d polarization function is added to the DZ basis set (except Hydrogen). From the notation " $6-31 \mathrm{~g}(\mathrm{~d})$ ", it can be inferred that one CGTO composed of 6 primitive GTOs is assigned to the core orbitals, for valence orbitals one CGTO consisting of 3 primitive GTOs is assigned to the first basis function and 1 primitive GTO is assigned to the second basis function. Figure 15 demonstrates the number of basis functions required for a water molecule with respect to the type of basis sets such as STO-3G, 3-31g, 6-31g(d), and 6-31g+(d). In this thesis 6-31g(d) basis set is used for DFT calculations in all papers. 


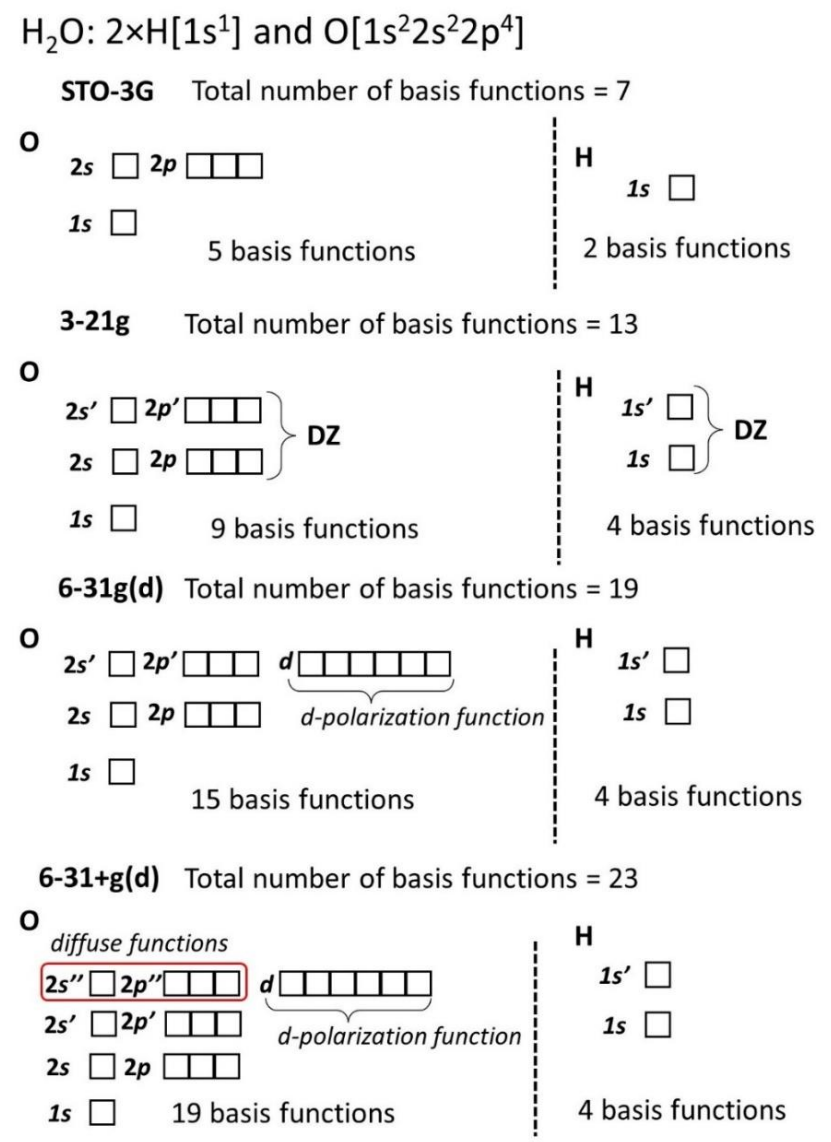

Figure 15 The number of basis functions in the basis sets (STO-3G,3$21 g, 6-31 g(d)$, and 6-31+(g)) required to describe a water molecule

\subsubsection{Solvent Effect}

In order to take the solvation effect into account, either an explicit model or implicit model can be exploited. The Polarizable Continuum model (PCM) ${ }^{119}$ is a commonly used implicit model in which the solvent is a uniform and polarizable medium with a fixed dielectric constant. In a dielectric medium, the solute molecules are placed inside the cavities that have a molecular shape of solute defined by overlapping atomic Van der Waals radii scaled by an empirical factor. Therefore, the charge density of the molecule in the cavity induces the polarization of dielectric medium with an apparent surface charge distribution on the cavity surface, resulting in a new potential term $\left(\widehat{V}_{\text {react }}\right)$ of the interaction between solute and solvent that is added to the electronic Hamiltonian in vacuo $\left(\widehat{H}_{o}\right)$. As a result, a new Schrodinger equation including solvation effect is introduced as follows, 


$$
\left[\widehat{H}_{o}+\widehat{V}_{\text {react }}\right] \psi=E \psi
$$

where,

$$
\widehat{V}_{\text {react }}(\vec{r})=\int \frac{\sigma_{S}(\vec{s})}{|\vec{r}-\vec{s}|} d^{2} s
$$

The term $\sigma_{s}(\vec{r})$ is an apparent surface charge (AFC) at a point $\vec{s}$ on the cavity surface, while $\vec{r}$ is the position of solute.

$$
\sigma_{S}(\vec{s})=\frac{\varepsilon-1}{4 \pi \varepsilon} \frac{\partial}{\partial \vec{n}}\left(\widehat{V}_{\text {solute }}(\vec{r})+\widehat{V}_{\text {react }}(\vec{r})\right)
$$

Equation 5.1.6.3

Here, $\widehat{V}_{\text {solute }}$ is the electrostatic potential arising from solute molecules, $\varepsilon$ is the dielectric constant of the solvent medium, and $\vec{n}$ is a unit vector perpendicular to the surface of cavity. In a practical calculation, the solute-solvent interaction potential $\left(\hat{V}_{\text {react }}\right)$ and apparent surface charge $\left(\sigma_{s}\right)$ could be self-consistently solved. The PCM model is used DFT calculations as implemented in Gaussian 09 package in paper I and II.

\subsubsection{Optimization}

Through exploring the potential energy of many-body systems (e.g., molecules) with respect to their coordinates, we can obtain an optimized geometry of the molecule. A typical geometry optimization includes repeating potential energy calculations and adjusting coordinates of atoms until reaching the (global) minimum on the potential energy surface (PES), which is repeating the following steps: 1) calculations of the potential energy and force $\left(-\frac{\partial E}{\partial \vec{r}}\right)$ of the initial structure of the molecule using DFT. 2) Subsequently, the geometry of molecule is updated by adding displacement to the coordinates of the nuclei (atoms) along the gradient of the potential energy reducing the force on the potential energy surface. 3) The potential energy and force of the updated geometry are calculated. The geometry with the minimum energy is obtained when the force equals zero (in practice, is less than a given threshold) and all elements of matrix of second derivative of potential energy $\left(\frac{\partial^{2} E}{\partial \vec{r}_{i} \vec{r}_{j}}\right)$ is positive. Therefore, it is important to choose a 'good' initial geometry of molecule to reduce computational cost for the geometry optimization. In all papers of the thesis, Berny Algorithm as implemented in Gaussian package is employed for all geometry optimizations.

\subsubsection{Thermodynamics Calculation}

Statistical Mechanics enables the connection between a macroscopic system and microscopic calculations. One can calculate the partition function $(q)$ of many-body system so as to estimate thermodynamical quantities of the system such as Gibbs free energy. Since the energy can be written as a sum of electronic, rotational, translational, and vibrational terms, the partition function can be divided into electronic, rotational, translational and vibrational functions according to the following equation.

$$
q_{\text {tot }}=q_{\text {trans }} q_{\text {rot }} q_{v i b} q_{\text {elec }}
$$

Equation 5.1.8.1 
The thermodynamic functions such as Enthalpy $(\mathrm{H})$ and entropy $(\mathrm{S})$ could be derived by using the partition function $\left(q_{t o t}\right)$ as below,

$$
\begin{gathered}
H=U+P V=k_{B} T^{2}\left(\frac{\partial \ln q}{\partial T}\right)_{V}+k_{B} T V\left(\frac{\partial \ln q}{\partial V}\right)_{T} \\
S=k_{B} T\left(\frac{\partial \ln q}{\partial T}\right)_{V}
\end{gathered}
$$

where, $U, P, V$, and $k_{B}$ are internal energy, pressure, volume and the Boltzmann's constant $\left(1.380658 \times 10^{-23} \mathrm{~J} / \mathrm{K}\right)$.

Inserting $q_{\text {tot }}$ into Equation 5.1.8.2 and Equation 5.1.8.3 results in Enthalpy $(H)$ and Entropy $(S)$ as a sum of each contributions defined as,

$$
\begin{gathered}
H_{\text {tot }}=H_{\text {trans }}+H_{\text {rot }}+H_{\text {vib }}+H_{\text {elec }} \\
S_{\text {tot }}=S_{\text {trans }}+S_{\text {rot }}+S_{\text {vib }}+S_{\text {elec }}
\end{gathered}
$$

Equation 5.1.8.4

Equation 5.1.8.5

Finally, the Gibbs free energy $(G)$ can be obtained by the following equations,

$$
G=H-T S
$$

Equation 5.1.8.6

In calculating the thermodynamical quantities, the vibrational contribution demands a calculation of all vibration frequencies (cf. for $\mathrm{N}$-particle system, $3 \mathrm{~N}-6$, (for linear molecules $3 N-5)$ ) that is another demanding calculation apart from the calculation of the potential energy in optimization. At the minimum point on the Potential Energy Surface (PES), i.e., for the optimized geometry, the vibrational frequency as well as vibration mode can be computed by diagonalization of the matrix of the second derivative of the potential energy, also called as Hessian matrix. 


\subsection{Molecular Dynamics}

\subsubsection{Introduction to Molecular dynamics}

Classical Molecular Dynamics (MD) ${ }^{120}$ is an efficient method to investigate the dynamics and trajectory of particles in a system by solving the Newton's equations of motion on the energy surface at finite temperature,

$$
F_{i}=-\frac{\partial V(r)}{\partial r_{i}}=m_{i} a=m_{i} \frac{d^{2} r_{i}}{d t^{2}}
$$

Equation 5.2.1.1

Here, $F_{i}$ is force on a particle $i$, and $V(r)$ denotes the potential energy. MD methods follow a typical procedure as shown in Figure 16: 1) Define initial coordinates of particles, 2) Set simulation conditions such as temperature $(\mathrm{T})$, pressure $(\mathrm{P})$, integration timesteps, simulation time (time steps) and so on, 3) assign initial (random) velocities to the particles according to the Maxwell-Boltzmann distribution, 4) calculate the forces acting on the particles by the derivative of the potential energy, 5) update the coordinates and velocities of particles by integrating Newton's equations of motion (Equation 5.2.1.1), 6) control the Temperature and Pressure as set in the beginning of simulation, and repeat step 4) -6) until the given simulation time in step 2) is reached.

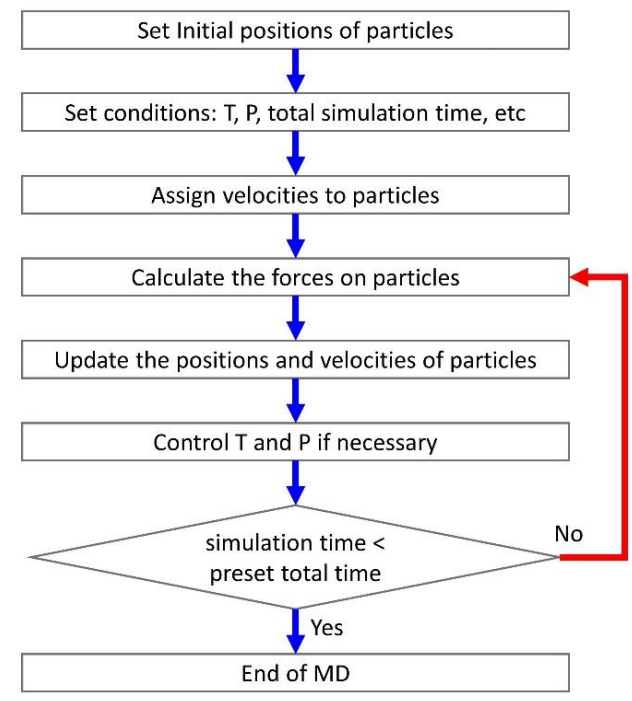

Figure 16 Flowchart of MD simulation

As described in the flowchart of MD simulations, the time in MD methods is discretized, and the simulation time could be defined as a multiple of the (integration) timestep $(\Delta \boldsymbol{t})$. Concomitantly, an integration algorithm is required to compute the time evolution of the positions and velocities of the particles. Therefore, a variety of integration methods such as Verlet method, ${ }^{121}$ leap-frog method, ${ }^{122}$ and velocity-Verlet method ${ }^{123}$ are developed and 
commonly used. ${ }^{120}$ The Verlet method is derived from two Taylor expansions of position $\boldsymbol{r}(\boldsymbol{t})$ in two time directions $\boldsymbol{r}(\boldsymbol{t} \pm \Delta \boldsymbol{t})$,

$$
\begin{aligned}
r(t+\Delta t)= & r(t)+\frac{d r(t)}{d t} \Delta t+\frac{1}{2} \frac{d^{2} r(t)}{d t^{2}} \Delta t^{2}+\frac{1}{3 !} \frac{d^{3} r(t)}{d t^{3}} \Delta t^{3} \\
& +O\left(\Delta t^{4}\right) \\
r(t-\Delta t)= & r(t)-\frac{d r(t)}{d t} \Delta t+\frac{1}{2} \frac{d^{2} r(t)}{d t^{2}} \Delta t^{2}-\frac{1}{3 !} \frac{d^{3} r(t)}{d t^{3}} \Delta t^{3} \\
& +O\left(\Delta t^{4}\right)
\end{aligned}
$$

Equation 5.2.1.2

Equation 5.2.1.3

where the second and third term are the velocity and acceleration, respectively. The fourth term is the third derivative of position.

The sum of Equation 5.2.1.2 and Equation 5.2.1.3 results in a simplified form of equation as below.

$$
r(t+\Delta t)=2 r(t)-r(t-\Delta t)+\frac{d^{2} r(t)}{d t^{2}} \Delta t^{2}+O\left(\Delta t^{4}\right)
$$

Equation 5.2.1.4

The velocity (Equation 5.2.1.5) can be obtained by subtracting Equation 5.2.1.3 from Equation 5.2.1.2.

$$
v(t)=\frac{r(t+\Delta t)-r(t-\Delta t)}{2 \Delta t}+O\left(\Delta t^{2}\right)
$$

Although the Verlet method is very simple and efficient, it has shortcomings: 1) the velocity is one step behind the position, 2) truncation error induced by adding $\Delta t^{2}$ to $2 r(t)-r(t-\Delta t)$. The velocity-Verlet integrator could solve these problems via employing Taylor expansions of both position and velocity as following equations,

$$
\begin{gathered}
r(t+\Delta t)=r(t)+v(t) \Delta t+\frac{1}{2} a(t) \Delta t^{2} \\
v(t+\Delta t)=v(t)+\frac{1}{2} \Delta t(a(t)+a(t+\Delta t))
\end{gathered}
$$

Equation 5.2.1.6

Equation 5.2.1.7

In the velocity-Verlet integrator, firstly the position at time $t+\Delta t$ is calculated and then the velocity is calculated by the velocity at $t+1 / 2 \Delta t$ (i.e., mid-step velocity) together with the acceleration at $t+\Delta t(a(t+\Delta t))$ computed from the force at $r(t+\Delta t)$. As a result, one can obtain the position and velocity at $t+\Delta t$ at the same time step. In this thesis, the velocityVerlet integrator as implemented in LAMMPS package is used for Paper II-V.

\subsubsection{Force Field}

Another key element in MD methods is to obtain the potential to calculate the forces acting on the particles. Classical MD methods exploit an empirical potential energy on which the accuracy of simulations considerably depends. A number of force fields (empirical potential) have been developed to describe potentials between particles in MD simulations.

The potential energy ( $V$ ) of the system can be divided into bonded and non-bonded interactions. The former can be further divided into bond, angle, dihedral angle, and improper dihedral angle terms, while the latter can be separated into Van der Waals $(v d w)$ and Coulombic terms, 


$$
\begin{gathered}
V_{\text {total }}=V_{\text {bond }}+V_{\text {angle }}+V_{\text {dihedral }}+V_{\text {improper }} \\
+V_{\text {vdw }}+V_{\text {coul }}
\end{gathered}
$$

where first four terms are bonded interactions, and the rest is non-bonded interactions. A conceptual scheme of bonded interactions in Equation 5.2.2.1 is illustrated in Figure 17. In Papers II, III, IV, V, the general amber force field (GAFF) ${ }^{124}$ is employed to carry out MD simulations.

Bond

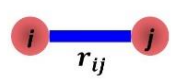

Dihedral

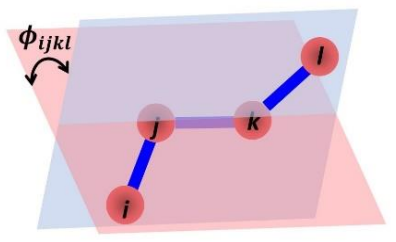

Angle
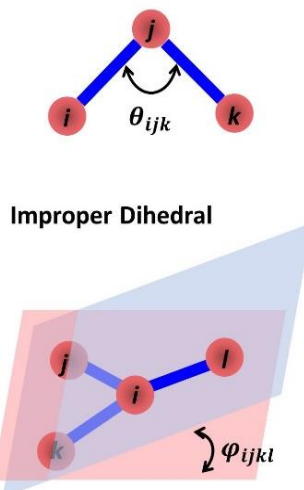

Figure 17 A conceptual illustrations of bonded potentials such as bond, angle, dihedral, improper dihedral. Note that the (improper or) dihedral is the angle between planes of ijk and jkl.

The equation for each term in GAFF force field could be expressed as follows.

\section{Harmonic potential for the bond potentials}

$$
V_{\text {bond }}=\sum_{\text {bonds }} k_{i j}^{r}\left(r_{i j}-r_{0}\right)^{2}
$$

$k_{i j}^{r}, r_{i j}$, and $r_{0}$ are the force constant, distance between particles $i$ and $j$, and equilibrium bond length respectively.

\section{Harmonic potential for angle potentials}

$$
V_{\text {angle }}=\sum_{\text {angles }} k_{i j k}^{\theta}\left(\theta_{i j k}-\theta_{0}\right)^{2}
$$

$k_{i j k}^{\theta}, \theta_{i j k}$, and $\theta_{0}$ are the force constant, angle between two bonds $\left(r_{i j}\right.$ and $r_{j k}$ for three consecutive particles $i, j$, and $k$ ), and the equilibrium angle respectively.

\section{Dihedral angle potential}

$$
V_{\text {dihedral }}=\sum_{\text {dihedrals }} k_{i j k l}^{\phi}\left[1+\cos \left(n \phi_{i j k l}-\phi_{0}\right)\right] \quad \text { Equation 5.2.2.4 }
$$

$k_{i j k l}^{\phi}, \phi_{i j k l}$, and $\phi_{0}$ the force constant, dihedral angle between two planes (formed by $i j k$ and $j k l$, while $i, j, k$, and $l$ are four consecutive particles), and equilibrium dihedral angle 
respectively. It should be noted that the dihedral angle $\phi_{i j k l}$ is multiplied by a positive integer $n$, indicating that the number of minima upon rotating the bond until $360^{\circ}$.

\section{Improper dihedral angle potential}

$$
V_{\text {improper }}=\sum_{\text {impropers }} k_{i j k l}^{\varphi}\left(\varphi_{i j k l}-\varphi_{0}\right)^{2}
$$

$k_{i j k l}^{\varphi}, \varphi_{i j k l}$, and $\varphi_{0}$ are the force constant, improper dihedral angle, and equilibrium improper dihedral angle respectively. The improper dihedral angle is defined by two planes obtained from ijk and jkl particles as shown in Figure 17.

\section{Lennard-Jones (LJ) potential (Van der Waals potential)}

$$
V_{v d w}=\sum_{i<j}\left(\frac{A_{i j}}{r_{i j}^{12}}-\frac{B_{i j}}{r_{i j}^{6}}\right)
$$

where $A_{i j}$ and $B_{i j}$ are the repulsion and attraction parameters that are $4 \epsilon \sigma^{12}$ and $4 \epsilon \sigma^{6}$ respectively ( $\epsilon$ and $\sigma$ are the depth of potential well and the equilibrium distance between particle $i$ and $j$ ). $r_{i j}$ is the distance between particles $i$ and $j$. It should be noted that the LJ parameters $\left(A_{i j}\right.$ and $\left.B_{i j}\right)$ between two different particles $i$ and $j$ can be obtained by the geometric mean of the parameters of each particle $\left(\sqrt{A_{i i} A_{j j}}\right.$ and $\left.\sqrt{B_{i i} B_{j j}}\right)$.

\section{Coulombic potential (Electrostatic potential)}

$$
V_{\text {coul }}=\sum_{i<j} \frac{q_{i} q_{j}}{4 \pi \varepsilon r_{i j}}
$$

$q_{i}, q_{j}$ are the charges of particle $i$ and $j$, and $\varepsilon$ and $r_{i j}$ is the dielectric constant and distance between particles $i$ and $j$.

A periodic boundary condition (PBC) allows one to study the bulk properties using a small part of a big system and remedy the edge effects of atoms near the boundary of the simulation box. The PBC could be achieved by replicating the simulation box to all directions as shown in Figure 18. In the PBC, particles in the simulation box and the copies are basically identical, and thereby when a particle of the simulation box passes through its boundary the particle reenters the box from the opposite boundary. 


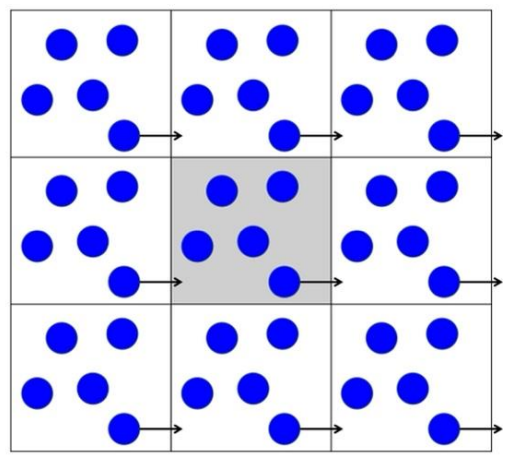

Figure 18 A conceptual scheme of Periodic Boundary Condition of a $2 D$ system. The box filled with grey color corresponds to the basic simulation box while the white boxes are the copies of it. The blue dots are particles in the system.

The calculation of non-bonded potentials between particles is computationally expensive since there exist many pairs between particles $\left(\mathrm{N}^{2}\right.$ for $\mathrm{N}$ particle system). To reduce the computational cost, it is common to employ a cutoff beyond which the LJ potential is negligible, while the electrostatic potential should be addressed by long range algorithms such as Ewald summation and $\mathrm{P}^{3} \mathrm{M}$ (particle-particle particle-mesh). ${ }^{125-128}$ In papers II-V, the $\mathrm{P}^{3} \mathrm{M}$ algorithm was used to compute the long-range electrostatic interactions.

\subsubsection{Statistical Ensembles}

The microscopic properties such as the positions and velocities of particles obtained from MD methods could be converted to macroscopic observables such as pressure, temperature, energy, and heat capacities that one can measure in experiments. The time average of macroscopic observables from MD simulations of the system obeying the ergodic hypothesis corresponds to the ensemble averages in statistical mechanics. An ensemble is a collection of all possible systems that have different microscopic states but demonstrate a same macroscopic state.

NVE (Microcanonical Ensemble): the number of particles (N), volume (V), and energy (E) are constant. The NVE ensemble is used in paper III-V.

NVT (Isothermal-Isochoric Ensemble): the number of particles (N), volume (V), and temperature are constant. The NVT ensemble is used in paper II-V.

NPT (Isothermal-Isobaric Ensemble): the number of particles (N), pressure (P), and temperature (T) are constant. The NPT ensemble is used in paper II-V.

NPH (Isoenthalpic-Isobaric Ensemble): the number of particles $(\mathrm{N})$, pressure $(\mathrm{P})$, and enthalpy $(\mathrm{H})$ are constant. The NPH ensemble is used in paper III.

To control the temperature and pressure, one should employ thermostat and barostat such as Nosé-Hoover Thermostat and barostat, ${ }^{129-131}$ Andersen thermostat, ${ }^{132}$ Langevin thermostat ${ }^{133,134}$ and others. A basic idea of such methods is to couple the system to a heat bath (i.e., thermostat) or pressure bath (i.e. barostat). 
Andersen thermostat: the system is coupled to a heat bath set to a desired temperature by a stochastic collision of randomly selected particles. The new velocities of selected particles are derived from Maxwell-Boltzmann distribution corresponding to the desired temperature. The frequency of stochastic collision defines the strength of the coupling to the heat bath. Andersen thermostat is very simple to implement, but the dynamics is not physical since the velocities are decorrelated.

Nosé-Hoover Thermostat: Instead of stochastic collisions on the system, an extended system with two additional degrees of freedom associated to a heat bath can be employed. This extended system consists of the simulation (real) system and a heat bath which is set to a desired temperature. Based on the extended Lagrangian approach, the extended Hamiltonian of system with $N$ particles is represented as below,

$$
\widehat{H}=\sum_{i=1}^{N} \frac{p_{i}^{2}}{2 m_{i}}+V(r)+\frac{\xi^{2} Q}{2}+g k_{B} T \ln s
$$

Equation 5.2.3.1

where $Q$ is an effective mass determining the strength of the thermostat, $p_{i}$ is momentum of particle $i$, and $\xi$ is a friction coefficient that is associated a heat bath degree of freedom $\left(p_{s}=\right.$ $\left.Q \frac{d s}{d t}\right)$ related to the conjugate momentum to scaling factor $s$. The scaling factor $(s)$ is the other heat bath degree of freedom, indicating the position of the heat bath to which the system is coupled. $g$ is the degree of freedom of the system ( $=3 N$, for $N$-particle system), and $k_{B}$ is the Boltzmann's constant. The equation of motion governing the time evolution of the positions and momenta of particles could be defined as,

$$
\begin{gathered}
\frac{d r_{i}}{d t}=\frac{p_{i}}{m_{i}} \\
m_{i} \frac{d^{2} r_{i}}{d t^{2}}=-\frac{V(r)}{\partial r_{i}}-\xi m_{i} v_{i} \\
\frac{d \xi}{d t}=\frac{1}{Q}\left(\sum_{i=1}^{N} \frac{p_{i}^{2}}{m_{i}}-g k_{B} T\right) \\
\xi=\frac{d(\ln s)}{d t}
\end{gathered}
$$

Equation 5.2.3.2

Equation 5.2.3.3

Equation 5.2.3.4

Equation 5.2.3.5

Employing Nosé-Hoover thermostat allows to generate a canonical ensemble (NVT) very well during molecular dynamics.

Langevin Thermostat: Large particles moves as though they are in a bath of smaller particles that works as an implicit heat bath. Large particles push smaller particle, resulting in a damping force to the momenta. Moreover, the smaller particles continuously and randomly jostle the large particles, giving rise to a stochastic noise term in the force. Therefore, the equation of motion for Langevin Thermostat can be presented as,

$$
m_{i} \frac{d^{2} r_{i}}{d t^{2}}=-\frac{V(r)}{\partial r_{i}}-m_{i} \gamma \frac{d r_{i}}{d t}+\sqrt{2 \gamma m_{i} k_{B} T} r_{i}(t)
$$


where the first, second, and third terms are the force calculated from particle interaction potentials, the damping force, and the stochastic noise respectively. $\gamma$ of damping force is the damping constant $\left(\mathrm{s}^{-1}\right.$, also called as friction coefficient).

Nosé-Hoover Barostat: Similar to the thermostat as described above, the system is coupled to a pressure bath by extended Lagrangian approach so as to control the pressure of the system via adjusting the volume of the system.

The Nosé-Hoover Thermostat was employed for NPT and NVT in papers II-V. The NoséHoover barostat is used for NPT in papers II-V as well as NPH in paper III. The Langevin thermostat is employed in paper III-V.

\subsubsection{Stress Tensor calculation}

For calculation of the elastic modulus, the stress tensor of atoms in response to applied strain is computed as defined,

$$
\sigma_{a b}=\frac{1}{V}\left(-m v_{a} v_{b}-W_{a b}\right)
$$

where $m$ and $v_{i}$ denotes the mass and velocity in the $i$ direction of atom, $V$ is the average volume; the first term is the kinetic energy contribution, and the second term is the virial contribution due to both intra- and inter-molecular interactions given as follows,

$$
\begin{aligned}
& W_{a b}=\frac{1}{2} \sum_{n=1}^{N_{\text {pair }}}\left(r_{1 a} F_{1 b}+r_{2 a} F_{2 b}\right) \\
& +\frac{1}{2} \sum_{n=1}^{N_{\text {bond }}}\left(r_{1 a} F_{1 b}+r_{2 a} F_{2 b}\right) \\
& +\frac{1}{3} \sum_{n=1}^{N_{\text {angle }}}\left(r_{1 a} F_{1 b}+r_{2 a} F_{2 b}+r_{3 a} F_{3 b}\right) \\
& +\frac{1}{4} \sum_{n=1}^{N_{\text {dinedral }}}\left(r_{1 a} F_{1 b}+r_{2 a} F_{2 b}+r_{3 a} F_{3 b}+r_{4 a} F_{4 b}\right) \\
& +\frac{1}{4} \sum_{n=1}^{N_{\text {improper }}}\left(r_{1 a} F_{1 b}+r_{2 a} F_{2 b}+r_{3 a} F_{3 b}+r_{4 a} F_{4 b}\right) \\
& + \text { Kspace }\left(r_{\text {ia }}, F_{i b}\right)
\end{aligned}
$$

Equation 5.2.4.2

In the virial contribution, the first term is a non-bonded pairwise energy contribution, the second, third, fourth, and fifth term are bond, angle, dihedral angle, and improper dihedral angle contribution, respectively. The seventh term is a contribution of the long-range Coulombic interactions. $r_{i}$ and $F_{i}$ are the position and force of the atom $i$ respectively in each term. The $N_{\text {pair }}, N_{\text {bond }}, N_{\text {angle }}, N_{\text {dinedral }}$, and $N_{\text {improper }}$ are the number of pairwise, bond, angle, dihedral, and improper interactions respectively. 


\section{Conclusion}

For fabrication of flexible or stretchable electric devices, it is essential to design a conducting polymer with well-adjusted mechanical properties as well as electrical conductivity. The charge-carrier concentration (doping level), chain length, morphology, and crystallinity of conducting polymers are crucial factors affecting their electrical and mechanical properties. The goal of the studies presented in the thesis was to explore two classes of conducting polymers: Thiophene-based and NDI-based polymers to provide detailed insight into the polymerization mechanism, morphology, and their mechanical properties.

\subsection{In-situ chemical polymerization mechanism and morphology of PEDOT:TOS}

Although enormous experiments have disclosed several aspects of PEDOT such as its pristine doping level (typically Ox. 33\%), relatively short chain length (10 20 monomer units), and polymerization mechanism, a theoretical understanding of these aspects is completely missing. In paper I, using Density Functional Theory, we verified the In-situ chemical polymerization (ICP) mechanism of PEDOT with Fe(TOS) $)_{3}$ from the point of view of thermodynamics and kinetics by the reaction energy calculation.

We also calculated the energy profile as function of the doping level for PEDOT $\mathrm{N}_{\mathrm{N}=6,9}$ and 12 (N denotes the length of PEDOT) to provide a theoretical understanding why pristine PEDOT is usually doped to $\mathrm{Ox}=33 \%$. Our results of energetics with respect to the doping level demonstrate that PEDOT with higher doping (oxidation) level than $\mathrm{Ox}=33 \%$ becomes

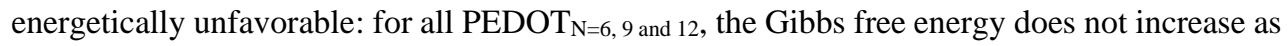
doping level increases from $\mathrm{Ox}=0$ to $33 \%$, but it subsequently strongly increases above $\mathrm{Ox}=$ $33 \%$. We associate this behaviour to the change in the structure of backbone from aromatic to quinoid, indicating the polaron localization in the backbone.

Our calculations showed that the length of PEDOT oligomers is not thermodynamically limited: the Gibbs free energy of PEDOT $\mathrm{N}$ linearly decreases as $\mathrm{N}$ increases. This result indicates that there should be other factors (not energetics) limiting the chain length to $10-20$ monomer units as observed by experimental studies.

The question about PEDOT chain lengths led us to the next project to investigate what the average PEDOT length is, and how it is affected by the polymerization temperature. In paper II, based on the DFT calculations of the polymerization energetics reported in Paper I, we developed a polymerization model using Molecular Dynamics simulations to study the ICP of PEDOT:TOS. In the polymerization model, reactants (EDOT monomer, PEDOT oligomers) create new bonds with a given probability when alpha carbons in each thiophene ring find other alpha carbons within a given radius. The polymerization simulations were carried out at three 
temperatures, and we found that the average chain length $(\langle N\rangle)$ is larger at higher temperature: $\langle N\rangle=6,7$ and 11 at $\mathrm{T}=298,323$ and $373 \mathrm{~K}$ respectively. We relate the temperature dependence of the average chain length to the diffusivity of reactants that is higher at $373 \mathrm{~K}$ than at 298 and 323K. The analysis of morphology of systems by XRD (X-Ray diffraction) and RDF (Radial Distribution Function) demonstrates that the formation of crystallites proceeds in the course of polymerization, resulting in the decrease in the diffusivity of oligomers as polymerization proceeds. With these results, we conclude that ICP of PEDOT:TOS is a diffusion-controlled reaction.

\subsection{Molecular doping effect on the electrical and mechanical properties of $p\left(g_{4} 2 T-T\right)$}

Since many conducting polymers, for example P3HT and PEDOT, ${ }^{135,136}$ are hard and brittle at room temperature, they need to be softened for flexible electric devices. On the other hand, one should render $\mathrm{p}\left(\mathrm{g}_{4} 2 \mathrm{~T}-\mathrm{T}\right)$ rigid since it is excessively soft at room temperature as its low glass transition temperature $\left(\mathrm{Tg} \approx-46^{\circ} \mathrm{C}\right)$ implies. Therefore, in Paper III we investigated the effect of molecular doping with TFSI dopant on the morphology of $\mathrm{p}\left(\mathrm{g}_{4} 2 \mathrm{~T}-\mathrm{T}\right)$ as well as on its conductivity.

The GIWAXS measurements show that no $\pi$ stacking forms at the doping level $\mathrm{Ox}=0 \%$. $\pi$ stacking appears and increases as TFSI concentration increases up to $\mathrm{Ox}=7.8 \%$, and subsequently reduces above $\mathrm{Ox}=22.2 \%$. This result is consistent with the corresponding MD simulations. Moreover, the calculated RDFs between polymers and counter anions reveal that the distance between them decreases as oxidation level increases from $\mathrm{Ox}=8.3$ to $33 \%$. From these results, we conclude that the interaction between polymers and counter anions plays a crucial role in the formation of $\pi$ stacking in the films: it helps to build the $\pi$ stacking for the oxidation levels from 3.7 to $7.8 \%$ like a glue, whereas above $\mathrm{Ox}=22.2 \%$ it is so strong that the formation of $\pi$ stacking is disturbed by counter anions situated too close to polymer chains. In addition, the conductivity of $\mathrm{p}\left(\mathrm{g}_{4} 2 \mathrm{~T}-\mathrm{T}\right)$ increases up to $57 \mathrm{Scm}^{-1}$ at $\mathrm{Ox}=33.3 \%$, and subsequently drops at higher oxidation level $(\mathrm{Ox}>33.3 \%)$. Therefore, we could conclude that the more ordered nanostructure induced by molecular doping gives rise to the increase in the conductivity.

Paper IV discusses the effect of molecular doping of $\mathrm{p}\left(\mathrm{g}_{4} 2 \mathrm{~T}-\mathrm{T}\right)$ with dopant F4TCNQ that is capable of doubly doping polymers, meaning that a F4TCNQ molecule can extract two electrons from polymers. ${ }^{84}$ The morphology, electrical and mechanical properties of $\mathrm{p}(\mathrm{g} 42 \mathrm{~T}$ T) for different oxidation levels were investigated by a combination of experiment and MD simulations. The morphology analysis by MD simulations indicates that the degree of crystallinity of $\mathrm{p}\left(\mathrm{g}_{4} 2 \mathrm{~T}-\mathrm{T}\right)$ is strongly improved as oxidation level increases from $\mathrm{Ox}=0$ to $16.7 \%$. The elastic modulus is enhanced by a factor of 29 (from 8 to $232 \mathrm{MPa}$ ) through molecular doping with F4TCNQ from $\mathrm{Ox}=0$ to $18.2 \%$, accompanied by the increase of conductivity up to $52 \mathrm{Scm}^{-1}$. The results of paper III and IV, demonstrate that that molecular 
doping is a powerful tool to adjust and tune the electrical and mechanical properties of $\mathrm{p}\left(\mathrm{g}_{4} 2 \mathrm{~T}\right.$ $\mathrm{T})$.

\subsection{Tuning the mechanical properties of PNDI-TVT-TET by controlling the $\pi$-conjugation length of chain}

Another strategy to modulate the mechanical properties of conducting polymer is to adjust the length of $\pi$ conjugation by incorporating $\pi$ nonconjugated units into the polymer chains. Paper $\mathrm{V}$ demonstrates that decreasing of the content of TVT ( $\pi$ conjugated unit) in pNDI-TVT TET $_{1-}$ $x$ could enhance the mobility variation of semiconducting films during bending test that is normalized mobility by the mobility before bending. This better mobility variation makes it feasible to fabricate flexible organic thin-film transistors (FOTFTs) with NDI-based polymer as a semiconductor layer. The FOTFT with TVT 60 and $80 \%$ films show higher mobility variation than FOTFT with TVT $100 \%$.

Using MD simulations, we demonstrate that the persistence length of pNDI-TVT ${ }_{\mathrm{x}}-\mathrm{TET}_{1-\mathrm{x}}$ increases as function of TVT content ( $\pi$ conjugated unit), which indicates the increase of the bending stiffness. In addition, the AFM measurements demonstrate that the films with $100 \%$ TVT content have a much higher elastic modulus than the films with lower TVT contents. A measurement of strain at which the films are damaged under tensile strain shows that the damage strain decreases as a function of the TVT content. Such results of bending stiffness, elastic modulus and damage strain led us to attribute the enhancement in mobility variation to a better flexibility induced by a reduction of the $\pi$ conjugation length of chains.

To further understand the better mobility variation of films with lower TVT content, we investigated the morphology of films as a function of the TVT content by both experiments and MD simulations. Both RDFs between NDI units obtained from MD simulations and GIWAXS analysis show that the degree of $\pi$ stacking in films changes with respect to TVT content: the highest degree of $\pi$ stacking is obtained at TVT 50\%. Also, the GIWAXS measurement of stretched film shows that when the TVT content is lower than $100 \%$, the $\pi$ stackings in films remains almost intact. This can be a reason for the better mobility variation of films with the TVT content lower than $100 \%$. 


\section{References}

(1) Louis, E.; Macdiarmid, A.; Chiang, H. K. Synthesis of Electrically Conducting Organic Polymers : Halogen Derivatives of Polyacetylene, $(\mathrm{CH}), .3$.

(2) Chiang, C. K.; Fincher, C. R.; Park, Y. W.; Heeger, A. J.; Shirakawa, H.; Louis, E. J.; Gau, S. C.; MacDiarmid, A. G. Electrical Conductivity in Doped Polyacetylene. Phys. Rev. Lett. 1977, 39 (17), 1098-1101. https://doi.org/10.1103/PhysRevLett.39.1098.

(3) Heeger, A. J. Semiconducting and Metallic Polymers: The Fourth Generation of Polymeric Materials. J. Phys. Chem. B 2001, 105 (36), 8475-8491. https://doi.org/10.1021/jp011611w.

(4) Kim, M.; Ryu, S. U.; Park, S. A.; Choi, K.; Kim, T.; Chung, D.; Park, T. Donor-AcceptorConjugated Polymer for High-Performance Organic Field-Effect Transistors: A Progress Report. Adv. Funct. Mater. 2020, 30 (20), 1904545. https://doi.org/10.1002/adfm.201904545.

(5) Ohayon, D.; Nikiforidis, G.; Savva, A.; Giugni, A.; Wustoni, S.; Palanisamy, T.; Chen, X.; Maria, I. P.; Di Fabrizio, E.; Costa, P. M. F. J.; McCulloch, I.; Inal, S. Biofuel Powered Glucose Detection in Bodily Fluids with an N-Type Conjugated Polymer. Nat. Mater. 2020, 19 (4), 456-463. https://doi.org/10.1038/s41563-019-0556-4.

(6) Kiefer, D.; Giovannitti, A.; Sun, H.; Biskup, T.; Hofmann, A.; Koopmans, M.; Cendra, C.; Weber, S.; Anton Koster, L. J.; Olsson, E.; Rivnay, J.; Fabiano, S.; McCulloch, I.; Müller, C. Enhanced N-Doping Efficiency of a Naphthalenediimide-Based Copolymer through Polar Side Chains for Organic Thermoelectrics. ACS Energy Lett. 2018, 3 (2), 278-285. https://doi.org/10.1021/acsenergylett.7b01146.

(7) Lee, S.; Kim, Y.; Wu, Z.; Lee, C.; Oh, S. J.; Luan, N. T.; Lee, J.; Jeong, D.; Zhang, K.; Huang, F.; Kim, T.-S.; Woo, H. Y.; Kim, B. J. Aqueous-Soluble Naphthalene Diimide-Based Polymer Acceptors for Efficient and Air-Stable All-Polymer Solar Cells. ACS Appl. Mater. Interfaces 2019, 11 (48), 45038-45047. https://doi.org/10.1021/acsami.9b13812.

(8) McNeill, R.; Siudak, R.; Wardlaw, J. H.; Weiss, D. E. Electronic Conduction in Polymers. I. The Chemical Structure of Polypyrrole. Aust. J. Chem. 1963, 16 (6), 1056-1075. https://doi.org/10.1071/ch9631056.

(9) Bolto, B. A.; Weiss, D. E. Electronic Conduction in Polymers. II. The Electrochemical Reduction of Polypyrrole at Controlled Potential. Aust. J. Chem. 1963, 16 (6), 1076-1089. https://doi.org/10.1071/ch9631076.

(10) Bolto, B. A.; McNeill, R.; Weiss, D. E. Electronic Conduction in Polymers. III. Electronic Properties of Polypyrrole. Aust. J. Chem. 1963, 16 (6), 1090-1103. https://doi.org/10.1071/ch9631090.

(11) Letheby, H. XXIX.-On the Production of a Blue Substance by the Electrolysis of Sulphate of Aniline. J. Chem. Soc. 1862, 15 (0), 161-163. https://doi.org/10.1039/JS8621500161.

(12) Jozefowicz, M.; Yu, L. T.; Belorgey, G.; Buvet, R. Conductivité Electronique et Propriétés Chimiques de Polyanilines Oligomères. J. Polym. Sci. Part C Polym. Symp. 1967, 16 (5), 2943-2954. https://doi.org/10.1002/polc.5070160548.

(13) Tourillon, G.; Garnier, F. New Electrochemically Generated Organic Conducting Polymers. $J$. Electroanal. Chem. Interfacial Electrochem. 1982, 135 (1), 173-178. https://doi.org/10.1016/00220728(82)90015-8. 
(14) Armour, M.; Davies, A. G.; Upadhyay, J.; Wassermann, A. Colored Electrically Conducting Polymers from Furan, Pyrrole, and Thiophene. J. Polym. Sci. [A1] 1967, 5 (7), 1527-1538. https://doi.org/10.1002/pol.1967.150050704.

(15) Peierls, R. E. Quantum Theory of Solids; Oxford University Press, 1996.

(16) Bredas, J. L.; Street, G. B. Polarons, Bipolarons, and Solitons in Conducting Polymers. Acc. Chem. Res. 1985, 18 (10), 309-315. https://doi.org/10.1021/ar00118a005.

(17) The Concept of 'Doping' of Conducting Polymers: The Role of Reduction Potentials. Philos. Trans. R. Soc. Lond. Ser. Math. Phys. Sci. 1985, 314 (1528), 3-15. https://doi.org/10.1098/rsta.1985.0004.

(18) Salzner, U. Electronic Structure of Conducting Organic Polymers: Insights from TimeDependent Density Functional Theory. WIREs Comput. Mol. Sci. 2014, 4 (6), 601-622. https://doi.org/10.1002/wcms.1194.

(19) Zozoulenko, I.; Singh, A.; Singh, S. K.; Gueskine, V.; Crispin, X.; Berggren, M. Polarons, Bipolarons, And Absorption Spectroscopy of PEDOT. ACS Appl. Polym. Mater. 2019, 1 (1), 83-94. https://doi.org/10.1021/acsapm.8b00061.

(20) Sahalianov, I.; Hynynen, J.; Barlow, S.; Marder, S. R.; Müller, C.; Zozoulenko, I. UV-to-IR Absorption of Molecularly p-Doped Polythiophenes with Alkyl and Oligoether Side Chains: Experiment and Interpretation Based on Density Functional Theory. J. Phys. Chem. B 2020, 124 (49), 11280-11293. https://doi.org/10.1021/acs.jpcb.0c08757.

(21) Zozoulenko, I.; Franco-Gonzalez, J. F.; Gueskine, V.; Mehandzhiyski, A.; Modarresi, M.; Rolland, N.; Tybrandt, K. Electronic, Optical, Morphological, Transport, and Electrochemical Properties of PEDOT: A Theoretical Perspective. Macromolecules 2021, 54 (13), 5915-5934. https://doi.org/10.1021/acs.macromol.1c00444.

(22) Haare, J. A. E. H. van; Havinga, E. E.; Dongen, J. L. J. van; Janssen, R. A. J.; Cornil, J.; Brédas, J.-L. Redox States of Long Oligothiophenes: Two Polarons on a Single Chain. Chem. - Eur. J. 1998, 4 (8), 1509-1522. https://doi.org/10.1002/(SICI)1521-3765(19980807)4:8<1509::AIDCHEM1509>3.0.CO;2-\#.

(23) Cornil, J.; Beljonne, D.; Brédas, J. L. Nature of Optical Transitions in Conjugated Oligomers. II. Theoretical Characterization of Neutral and Doped Oligothiophenes. J. Chem. Phys. 1995, 103 (2), 842-849. https://doi.org/10.1063/1.470065.

(24) Stafström, S.; Brédas, J. L. Evolution of the Electronic Structure of Polyacetylene and Polythiophene as a Function of Doping Level and Lattice Conformation. Phys. Rev. B 1988, 38 (6), 4180-4191. https://doi.org/10.1103/PhysRevB.38.4180.

(25) Ghosh, S.; Gueskine, V.; Berggren, M.; Zozoulenko, I. V. Electronic Structures and Optical Absorption of N-Type Conducting Polymers at Different Doping Levels. J. Phys. Chem. C 2019, 123 (25), 15467-15476. https://doi.org/10.1021/acs.jpcc.9b04634.

(26) Su, W. P.; Schrieffer, J. R. Soliton Dynamics in Polyacetylene. Proc. Natl. Acad. Sci. 1980, 77 (10), 5626-5629. https://doi.org/10.1073/pnas.77.10.5626.

(27) Le, T.-H.; Kim, Y.; Yoon, H. Electrical and Electrochemical Properties of Conducting Polymers. Polymers 2017, 9 (12), 150. https://doi.org/10.3390/polym9040150. 
(28) Rolland, N.; Franco-Gonzalez, J. F.; Volpi, R.; Linares, M.; Zozoulenko, I. V. Understanding Morphology-Mobility Dependence in PEDOT:Tos. Phys. Rev. Mater. 4, 2 (4), 045605. https://doi.org/10.1103/PhysRevMaterials.2.045605.

(29) Petsagkourakis, I.; Pavlopoulou, E.; Portale, G.; Kuropatwa, B. A.; Dilhaire, S.; Fleury, G.; Hadziioannou, G. Structurally-Driven Enhancement of Thermoelectric Properties within Poly(3,4Ethylenedioxythiophene) Thin Films. Sci. Rep. 2016, 6, 30501. https://doi.org/10.1038/srep30501.

(30) Ghosh, S.; Rolland, N.; Zozoulenko, I. Electronic Structure, Optical Properties, Morphology and Charge Transport in Naphthalenediimide (NDI)-Based n-Type Copolymer with Altered $\pi$ Conjugation: A Theoretical Perspective. Appl. Phys. Lett. 2021, 118 (22), 223302. https://doi.org/10.1063/5.0051166.

(31) Heydari Gharahcheshmeh, M.; Gleason, K. K. Texture and Nanostructural Engineering of Conjugated Conducting and Semiconducting Polymers. Mater. Today Adv. 2020, 8, 100086. https://doi.org/10.1016/j.mtadv.2020.100086.

(32) Jonas, F. D. C. D.; Heywang, G. D. C. D.; Schmidtberg, W. Novel Polythiophenes, Process for Their Preparation, and Their Use. DE3813589A1, November 2, 1989.

(33) Jonas, F.; Schrader, L. Conductive Modifications of Polymers with Polypyrroles and Polythiophenes. Synth. Met. 1991, 41 (3), 831-836. https://doi.org/10.1016/0379-6779(91)91506-6.

(34) Heywang, G.; Jonas, F. Poly(Alkylenedioxythiophene)s-New, Very Stable Conducting Polymers. Adv. Mater. 1992, 4 (2), 116-118. https://doi.org/10.1002/adma.19920040213.

(35) Cho, B.; Park, K. S.; Baek, J.; Oh, H. S.; Koo Lee, Y.-E.; Sung, M. M. Single-Crystal Poly(3,4Ethylenedioxythiophene) Nanowires with Ultrahigh Conductivity. Nano Lett. 2014, 14 (6), 3321-3327. https://doi.org/10.1021/nl500748y.

(36) Berggren, M.; Crispin, X.; Fabiano, S.; Jonsson, M. P.; Simon, D. T.; Stavrinidou, E.; Tybrandt, K.; Zozoulenko, I. Ion Electron-Coupled Functionality in Materials and Devices Based on Conjugated Polymers. Adv. Mater. 2019, 31 (22), 1805813. https://doi.org/10.1002/adma.201805813.

(37) Petsagkourakis, I.; Kim, N.; Tybrandt, K.; Zozoulenko, I.; Crispin, X. Poly(3,4Ethylenedioxythiophene): Chemical Synthesis, Transport Properties, and Thermoelectric Devices. Adv. Electron. Mater. 2019, 5 (11), 1800918. https://doi.org/10.1002/aelm.201800918.

(38) Samba, R. Is the Enhanced Adhesion of PEDOT Thin Films on Electrodes Due to Sulfur - Gold Interaction? - An XPS Study. Open Surf. Sci. J. 2013, 5 (1), 17-20. https://doi.org/10.2174/1876531901305010017.

(39) Brooke, R.; Evans, D.; Dienel, M.; Hojati-Talemi, P.; Murphy, P.; Fabretto, M. Inkjet Printing and Vapor Phase Polymerization: Patterned Conductive PEDOT for Electronic Applications. J. Mater. Chem. C 2013, 1 (20), 3353-3358. https://doi.org/10.1039/C3TC30356J.

(40) Brooke, R.; Cottis, P.; Talemi, P.; Fabretto, M.; Murphy, P.; Evans, D. Recent Advances in the Synthesis of Conducting Polymers from the Vapour Phase. Prog. Mater. Sci. 2017, 86, 127-146. https://doi.org/10.1016/j.pmatsci.2017.01.004.

(41) Chen, S.; Petsagkourakis, I.; Spampinato, N.; Kuang, C.; Liu, X.; Brooke, R.; Kang, E. S. H.; Fahlman, M.; Crispin, X.; Pavlopoulou, E.; Jonsson, M. P. Unraveling Vertical Inhomogeneity in Vapour Phase Polymerized PEDOT:Tos Films. J. Mater. Chem. A 2020, 8 (36), 18726-18734. https://doi.org/10.1039/D0TA06031C. 
(42) Lock, J. P.; Im, S. G.; Gleason, K. K. Oxidative Chemical Vapor Deposition of Electrically Conducting Poly(3,4-Ethylenedioxythiophene) Films. Macromolecules 2006, 39 (16), 5326-5329. https://doi.org/10.1021/ma060113o.

(43) Culebras, M.; Gómez, C. M.; Cantarero, A. Enhanced Thermoelectric Performance of PEDOT with Different Counter-Ions Optimized by Chemical Reduction. J. Mater. Chem. A 2014, 2 (26), 1010910115. https://doi.org/10.1039/C4TA01012D.

(44) Akoudad, S.; Roncali, J. Chemical Reduction. Synth. Met. 1998, 93 (2), 111-114. https://doi.org/10.1016/S0379-6779(97)04100-3.

(45) Donahue, M. J.; Sanchez-Sanchez, A.; Inal, S.; Qu, J.; Owens, R. M.; Mecerreyes, D.; Malliaras, G. G.; Martin, D. C. Tailoring PEDOT Properties for Applications in Bioelectronics. Mater. Sci. Eng. R Rep. 2020, 140, 100546. https://doi.org/10.1016/j.mser.2020.100546.

(46) Paradee, N.; Sirivat, A. Synthesis of Poly(3,4-Ethylenedioxythiophene) Nanoparticles via Chemical Oxidation Polymerization. Polym. Int. 2014, 63 (1), 106-113. https://doi.org/10.1002/pi.4538.

(47) Edberg, J.; Inganäs, O.; Engquist, I.; Berggren, M. Boosting the Capacity of All-Organic Paper Supercapacitors Using Wood Derivatives. J. Mater. Chem. A 2018, 6 (1), 145-152. https://doi.org/10.1039/C7TA06810G.

(48) Sun, K.; Zhang, S. P.; Li, P. C.; Xia, Y. J.; Zhang, X.; Du, D. H.; Isikgor, F. H.; Ouyang, J. Y. Review on Application of PEDOTs and PEDOT: PSS in Energy Conversion and Storage Devices. $J$. Mater. Sci.-Mater. Electron. 2015, 26 (7), 4438-4462. https://doi.org/10.1007/s10854-015-2895-5.

(49) Gao, X.; Zu, L.; Cai, X.; Li, C.; Lian, H.; Liu, Y.; Wang, X.; Cui, X. High Performance of Supercapacitor from PEDOT:PSS Electrode and Redox Iodide Ion Electrolyte. Nanomaterials 2018, 8 (5). https://doi.org/10.3390/nano8050335.

(50) Li, B.; Lopez-Beltran, H.; Siu, C.; Skorenko, K. H.; Zhou, H.; Bernier, W. E.; Whittingham, M. S.; Jones, W. E. Vaper Phase Polymerized PEDOT/Cellulose Paper Composite for Flexible Solid-State Supercapacitor. ACS Appl. Energy Mater. 2020, 3 (2), 1559-1568. https://doi.org/10.1021/acsaem.9b02044.

(51) Isaksson, J.; Kjäll, P.; Nilsson, D.; Robinson, N.; Berggren, M.; Richter-Dahlfors, A. Electronic Control of Ca2+ Signalling in Neuronal Cells Using an Organic Electronic Ion Pump. Nat. Mater. 2007, 6, 673. https://doi.org/10.1038/nmat1963.

(52) Williamson, A.; Rivnay, J.; Kergoat, L.; Jonsson, A.; Inal, S.; Uguz, I.; Ferro, M.; Ivanov, A.; Sjöström, T. A.; Simon, D. T.; Berggren, M.; Malliaras, G. G.; Bernard, C. Controlling Epileptiform Activity with Organic Electronic Ion Pumps. 2015, 27 (20), 3138-3144. https://doi.org/10.1002/adma.201500482.

(53) Wijeratne, K.; Vagin, M.; Brooke, R.; Crispin, X. Poly(3,4-Ethylenedioxythiophene)-Tosylate (PEDOT-Tos) Electrodes in Thermogalvanic Cells. J. Mater. Chem. A 2017, 5 (37), 19619-19625. https://doi.org/10.1039/C7TA04891B.

(54) Ni, D.; Song, H.; Chen, Y.; Cai, K. Free-Standing Highly Conducting PEDOT Films for Flexible Thermoelectric Generator. Energy 2019, 170, 53-61. https://doi.org/10.1016/j.energy.2018.12.124.

(55) Kim, N.; Lienemann, S.; Petsagkourakis, I.; Alemu Mengistie, D.; Kee, S.; Ederth, T.; Gueskine, V.; Leclère, P.; Lazzaroni, R.; Crispin, X.; Tybrandt, K. Elastic Conducting Polymer Composites in Thermoelectric Modules. Nat. Commun. 2020, 11 (1), 1-10. https://doi.org/10.1038/s41467-02015135-w. 
(56) Lee, S. J. Organic Photovoltaic with PEDOT_PSS and V2O5 Mixture as Hole Transport Layer. Sol. Energy Mater. 2014, 6.

(57) Xu, B.; Gopalan, S.-A.; Gopalan, A.-I.; Muthuchamy, N.; Lee, K.-P.; Lee, J.-S.; Jiang, Y.; Lee, S.-W.; Kim, S.-W.; Kim, J.-S.; Jeong, H.-M.; Kwon, J.-B.; Bae, J.-H.; Kang, S.-W. Functional Solid Additive Modified PEDOT:PSS as an Anode Buffer Layer for Enhanced Photovoltaic Performance and Stability in Polymer Solar Cells. Sci. Rep. 2017, 7 (1), 45079. https://doi.org/10.1038/srep45079.

(58) Miglbauer, E.; Wójcik, P. J.; Głowacki, E. D. Single-Compartment Hydrogen Peroxide Fuel Cells with Poly(3,4-Ethylenedioxythiophene) Cathodes. Chem. Commun. 2018, 54 (84), 11873-11876. https://doi.org/10.1039/C8CC06802J.

(59) Singh, S. K.; Crispin, X.; Zozoulenko, I. V. Oxygen Reduction Reaction in Conducting Polymer PEDOT: Density Functional Theory Study. J. Phys. Chem. C 2017, 121 (22), 12270-12277. https://doi.org/10.1021/acs.jpcc.7b03210.

(60) Winther-Jensen, B.; Winther-Jensen, O.; Forsyth, M.; MacFarlane, D. R. High Rates of Oxygen Reduction over a Vapor Phase-Polymerized PEDOT Electrode. Science 2008, 321 (5889), 671-674. https://doi.org/10.1126/science.1159267.

(61) Brooke, R.; Edberg, J.; Crispin, X.; Berggren, M.; Engquist, I.; Jonsson, M. P. \%J P. Greyscale and Paper Electrochromic Polymer Displays by UV Patterning. 2019, 11 (2), 267.

(62) Cao, Y.; Yu, G.; Zhang, C.; Menon, R.; Heeger, A. J. Polymer Light-Emitting Diodes with Polyethylene Dioxythiophene-Polystyrene Sulfonate as the Transparent Anode. Synth. Met. 1997, 87 (2), 171-174. https://doi.org/10.1016/S0379-6779(97)03823-X.

(63) Stutzmann, N.; Friend, R. H.; Sirringhaus, H. Self-Aligned, Vertical-Channel, Polymer FieldEffect Transistors. Science 2003, 299 (5614), 1881. https://doi.org/10.1126/science.1081279.

(64) Yan, H.; Kagata, T.; Okuzaki, H. Micrometer-Scaled OFET Channel Patterns Fabricated by Using PEDOT/PSS Microfibers. Synth. Met. 2009, 159 (21), 2229-2232. https://doi.org/10.1016/j.synthmet.2009.07.033.

(65) Gunaydin, O.; Demir, A.; Demir, G. E.; Yücedağ, İ.; Çoşut, B. \%J M. R. The Preparation of Transparent Organic Field Effect Transistor Using a Novel EDOT Functional Styrene Copolymer Insulator With a PEDOT:PSS Gate Electrode. 2018, 26 (2), 164-172. https://doi.org/10.1007/s13233018-6023-2.

(66) Dkhissi, A.; Louwet, F.; Groenendaal, L.; Beljonne, D.; Lazzaroni, R.; Brédas, J. L. Theoretical Investigation of the Nature of the Ground State in the Low-Bandgap Conjugated Polymer, Poly $(3,4-$ Ethylenedioxythiophene). Chem. Phys. Lett. 2002, 359 (5), 466-472. https://doi.org/10.1016/S00092614(02)00651-6.

(67) Lang, U.; Müller, E.; Naujoks, N.; Dual, J. Microscopical Investigations of PEDOT:PSS Thin Films. Adv. Funct. Mater. 2009, 19 (8), 1215-1220. https://doi.org/10.1002/adfm.200801258.

(68) Kim, N.; Kee, S.; Lee, S. H.; Lee, B. H.; Kahng, Y. H.; Jo, Y.-R.; Kim, B.-J.; Lee, K. Highly Conductive PEDOT:PSS Nanofibrils Induced by Solution-Processed Crystallization. Adv. Mater. 2014, 26 (14), 2268-2272. https://doi.org/10.1002/adma.201304611.

(69) Khodakarimi, S.; Hekhmatshoar, M. H.; Nasiri, M.; Khaleghi Moghaddam, M.; Abbasi, F. Effects of Process and Post-Process Treatments on the Electrical Conductivity of the PEDOT:PSS Films. J. Mater. Sci. Mater. Electron. 2016, 27 (2), 1278-1285. https://doi.org/10.1007/s10854-015-3886-2. 
(70) Bubnova, O.; Khan, Z. U.; Wang, H.; Braun, S.; Evans, D. R.; Fabretto, M.; Hojati-Talemi, P.; Dagnelund, D.; Arlin, J.-B.; Geerts, Y. H.; Desbief, S.; Breiby, D. W.; Andreasen, J. W.; Lazzaroni, R.; Chen, W. M.; Zozoulenko, I.; Fahlman, M.; Murphy, P. J.; Berggren, M.; Crispin, X. Semi-Metallic Polymers. Nat. Mater. 2013, 13, 190. https://doi.org/10.1038/nmat3824.

(71) Aasmundtveit, K. E.; Samuelsen, E. J.; Pettersson, L. A. A.; Inganäs, O.; Johansson, T.; Feidenhans'l, R. Structure of Thin Films of Poly(3,4-Ethylenedioxythiophene). Synth. Met. 1999, 101 (1), 561-564. https://doi.org/10.1016/S0379-6779(98)00315-4.

(72) Perrot, S.; Pawula, F.; Pechev, S.; Hadziioannou, G.; Fleury, G. PEDOT:Tos Electronic and Thermoelectric Properties: Lessons from Two Polymerization Processes. J. Mater. Chem. C 2021, 9 (23), 7417-7425. https://doi.org/10.1039/D1TC00756D.

(73) Franco-Gonzalez, J. F.; Rolland, N.; Zozoulenko, I. V. Substrate-Dependent Morphology and Its Effect on Electrical Mobility of Doped Poly(3,4-Ethylenedioxythiophene) (PEDOT) Thin Films. ACS Appl. Mater. Interfaces 2018, 10 (34), 29115-29126. https://doi.org/10.1021/acsami.8b08774.

(74) Franco-Gonzalez, J. F.; Zozoulenko, I. V. Molecular Dynamics Study of Morphology of Doped PEDOT: From Solution to Dry Phase. J. Phys. Chem. B 2017, 121 (16), 4299-4307. https://doi.org/10.1021/acs.jpcb.7b01510.

(75) Gallazzi, M. C.; Tassoni, L.; Bertarelli, C.; Pioggia, G.; Di Francesco, F.; Montoneri, E. Poly(Alkoxy-Bithiophenes) Sensors for Organic Vapours. Sens. Actuators B Chem. 2003, 88 (2), 178189. https://doi.org/10.1016/S0925-4005(02)00323-4.

(76) Gladisch, J.; Stavrinidou, E.; Ghosh, S.; Giovannitti, A.; Moser, M.; Zozoulenko, I.; McCulloch, I.; Berggren, M. Reversible Electronic Solid-Gel Switching of a Conjugated Polymer. Adv. Sci. 2020, 7 (2), 1901144. https://doi.org/10.1002/advs.201901144.

(77) Moser, M.; Gladisch, J.; Ghosh, S.; Hidalgo, T. C.; Ponder, J. F.; Sheelamanthula, R.; Thiburce, Q.; Gasparini, N.; Wadsworth, A.; Salleo, A.; Inal, S.; Berggren, M.; Zozoulenko, I.; Stavrinidou, E.; McCulloch, I. Controlling Electrochemically Induced Volume Changes in Conjugated Polymers by Chemical Design: From Theory to Devices. Adv. Funct. Mater. 2021, 31 (26), 2100723. https://doi.org/10.1002/adfm.202100723.

(78) Moser, M.; Ponder, J. F.; Wadsworth, A.; Giovannitti, A.; McCulloch, I. Materials in Organic Electrochemical Transistors for Bioelectronic Applications: Past, Present, and Future. Adv. Funct. Mater. 2019, 29 (21), 1807033. https://doi.org/10.1002/adfm.201807033.

(79) Moser, M.; Hidalgo, T. C.; Surgailis, J.; Gladisch, J.; Ghosh, S.; Sheelamanthula, R.; Thiburce, Q.; Giovannitti, A.; Salleo, A.; Gasparini, N.; Wadsworth, A.; Zozoulenko, I.; Berggren, M.; Stavrinidou, E.; Inal, S.; McCulloch, I. Side Chain Redistribution as a Strategy to Boost Organic Electrochemical Transistor Performance and Stability. Adv. Mater. 2020, 32 (37), 2002748. https://doi.org/10.1002/adma.202002748.

(80) Zokaei, S.; Kroon, R.; Gladisch, J.; Paulsen, B. D.; Sohn, W.; Hofmann, A. I.; Persson, G.; Stamm, A.; Syrén, P.; Olsson, E.; Rivnay, J.; Stavrinidou, E.; Lund, A.; Müller, C. Toughening of a Soft Polar Polythiophene through Copolymerization with Hard Urethane Segments. Adv. Sci. 2021, 8 (2), 2002778. https://doi.org/10.1002/advs.202002778.

(81) Hofmann, A. I.; Kroon, R.; Yu, L.; Müller, C. Highly Stable Doping of a Polar Polythiophene through Co-Processing with Sulfonic Acids and Bistriflimide. J. Mater. Chem. C 2018, 6 (26), 69056910. https://doi.org/10.1039/C8TC01593G.

(82) Jacobs, I. E.; Aasen, E. W.; Oliveira, J. L.; Fonseca, T. N.; Roehling, J. D.; Li, J.; Zhang, G.; Augustine, M. P.; Mascal, M.; Moulé, A. J. Comparison of Solution-Mixed and Sequentially Processed 
P3HT:F4TCNQ Films: Effect of Doping-Induced Aggregation on Film Morphology. J Mater Chem C 2016, 4 (16), 3454-3466. https://doi.org/10.1039/C5TC04207K.

(83) Kroon, R.; Kiefer, D.; Stegerer, D.; Yu, L.; Sommer, M.; Müller, C. Polar Side Chains Enhance Processability, Electrical Conductivity, and Thermal Stability of a Molecularly p-Doped Polythiophene. Adv. Mater. 2017, 29 (24), 1700930. https://doi.org/10.1002/adma.201700930.

(84) Kiefer, D.; Kroon, R.; Hofmann, A. I.; Sun, H.; Liu, X.; Giovannitti, A.; Stegerer, D.; Cano, A.; Hynynen, J.; Yu, L.; Zhang, Y.; Nai, D.; Harrelson, T. F.; Sommer, M.; Moulé, A. J.; Kemerink, M.; Marder, S. R.; McCulloch, I.; Fahlman, M.; Fabiano, S.; Müller, C. Double Doping of Conjugated Polymers with Monomer Molecular Dopants. Nat. Mater. 2019, 18 (2), 149-155. https://doi.org/10.1038/s41563-018-0263-6.

(85) Kranthiraja, K.; Long, D. X.; Sree, V. G.; Cho, W.; Cho, Y.-R.; Zaheer, A.; Lee, J.-C.; Noh, Y.-Y.; Jin, S.-H. Sequential Fluorination on Napthaleneamide-Based Conjugated Polymers and Their Impact on Charge Transport Properties. Macromolecules 2018, 51 (15), 5530-5536. https://doi.org/10.1021/acs.macromol.8b00748.

(86) Erdmann, T.; Fabiano, S.; Milián-Medina, B.; Hanifi, D.; Chen, Z.; Berggren, M.; Gierschner, J.; Salleo, A.; Kiriy, A.; Voit, B.; Facchetti, A. Naphthalenediimide Polymers with Finely Tuned InChain $\pi$-Conjugation: Electronic Structure, Film Microstructure, and Charge Transport Properties. Adv. Mater. 2016, 28 (41), 9169-9174. https://doi.org/10.1002/adma.201602923.

(87) Nava, D.; Shin, Y.; Massetti, M.; Jiao, X.; Biskup, T.; Jagadeesh, M. S.; Calloni, A.; Duò, L.; Lanzani, G.; McNeill, C. R.; Sommer, M.; Caironi, M. Drastic Improvement of Air Stability in an NType Doped Naphthalene-Diimide Polymer by Thionation. ACS Appl. Energy Mater. 2018, 1 (9), 46264634. https://doi.org/10.1021/acsaem.8b00777.

(88) Tian, Z.; Li, J.; Li, C.; Jiang, X.; Guo, Y.; Xiao, C.; Li, W. A Naphthalenediimide-Based Polymer Acceptor with Multidirectional Orientations via Double-Cable Design. Macromolecules 2020, 53 (21), 9279-9286. https://doi.org/10.1021/acs.macromol.0c01952.

(89) Kang, B.; Kim, R.; Lee, S. B.; Kwon, S.-K.; Kim, Y.-H.; Cho, K. Side-Chain-Induced Rigid Backbone Organization of Polymer Semiconductors through Semifluoroalkyl Side Chains. J. Am. Chem. Soc. 2016, 138 (11), 3679-3686. https://doi.org/10.1021/jacs.5b10445.

(90) Elschner, A.; Kirchmeyer, S.; Lovenich, W.; Merker, U.; Reuter, K. PEDOT: Principles and Applications of an Intrinsically Conductive Polymer; CRC Press, 2010.

(91) Kirchmeyer, S.; Reuter, K. Scientific Importance, Properties and Growing Applications of Poly(3,4-Ethylenedioxythiophene). J. Mater. Chem. 2005, $15 \quad$ (21), 2077-2088. https://doi.org/10.1039/B417803N.

(92) Ha, Y. H.; Nikolov, N.; Pollack, S. K.; Mastrangelo, J.; Martin, B. D.; Shashidhar, R. Towards a Transparent, Highly Conductive Poly(3,4-Ethylenedioxythiophene). Adv. Funct. Mater. 2004, 14 (6), 615-622. https://doi.org/10.1002/adfm.200305059.

(93) Angell, C. A. Formation of Glasses from Liquids and Biopolymers. Science 1995, 267 (5206), 1924-1935. https://doi.org/10.1126/science.267.5206.1924.

(94) Dalle-Ferrier, C.; Kisliuk, A.; Hong, L.; Carini, G.; Carini, G.; D’Angelo, G.; Alba-Simionesco, C.; Novikov, V. N.; Sokolov, A. P. Why Many Polymers Are so Fragile: A New Perspective. J. Chem. Phys. 2016, 145 (15), 154901. https://doi.org/10.1063/1.4964362.

(95) Novikov, V. N.; Sokolov, A. P. Poisson's Ratio and the Fragility of Glass-Forming Liquids. Nature 2004, 431 (7011), 961-963. https://doi.org/10.1038/nature02947. 
(96) Novikov, V. N.; Ding, Y.; Sokolov, A. P. Correlation of Fragility of Supercooled Liquids with Elastic Properties of Glasses. Phys. Rev. $E$ 2005, 71 (6), 061501. https://doi.org/10.1103/PhysRevE.71.061501.

(97) Ciarella, S.; Biezemans, R. A.; Janssen, L. M. C. Understanding, Predicting, and Tuning the Fragility of Vitrimeric Polymers. Proc. Natl. Acad. Sci. 2019, 116 (50), 25013-25022. https://doi.org/10.1073/pnas.1912571116.

(98) Araujo, S.; Batteux, F.; Li, W.; Butterfield, L.; Delpouve, N.; Esposito, A.; Tan, L.; Saiter, J.M.; Negahban, M. A Structural Interpretation of the Two Components Governing the Kinetic Fragility from the Example of Interpenetrated Polymer Networks: A Structural Interpretation of the Two Components Governing the Kinetic Fragility from the Example of Interpenetrated Polymer Networks. J. Polym. Sci. Part B Polym. Phys. 2018, 56 (20), 1393-1403. https://doi.org/10.1002/polb.24722.

(99) Qin, Q.; McKenna, G. B. Correlation between Dynamic Fragility and Glass Transition Temperature for Different Classes of Glass Forming Liquids. J. Non-Cryst. Solids 2006, 352 (28-29), 2977-2985. https://doi.org/10.1016/j.jnoncrysol.2006.04.014.

(100) Alves, N. M.; Gómez Ribelles, J. L.; Mano, J. F. Enthalpy Relaxation Studies in Polymethyl Methacrylate Networks with Different Crosslinking Degrees. Polymer 2005, 46 (2), 491-504. https://doi.org/10.1016/j.polymer.2004.11.016.

(101) Tummala, N. R.; Bruner, C.; Risko, C.; Brédas, J.-L.; Dauskardt, R. H. Molecular-Scale Understanding of Cohesion and Fracture in P3HT:Fullerene Blends. ACS Appl. Mater. Interfaces 2015, 7 (18), 9957-9964. https://doi.org/10.1021/acsami.5b02202.

(102) Rodriquez, D.; Kim, J.-H.; Root, S. E.; Fei, Z.; Boufflet, P.; Heeney, M.; Kim, T.-S.; Lipomi, D. J. Comparison of Methods for Determining the Mechanical Properties of Semiconducting Polymer Films for Stretchable Electronics. ACS Appl. Mater. Interfaces 2017, 9 (10), 8855-8862. https://doi.org/10.1021/acsami.6b16115.

(103) Savagatrup, S.; Makaram, A. S.; Burke, D. J.; Lipomi, D. J. Mechanical Properties of Conjugated Polymers and Polymer-Fullerene Composites as a Function of Molecular Structure. Adv. Funct. Mater. 2014, 24 (8), 1169-1181. https://doi.org/10.1002/adfm.201302646.

(104) Kim, J.-S.; Kim, J.-H.; Lee, W.; Yu, H.; Kim, H. J.; Song, I.; Shin, M.; Oh, J. H.; Jeong, U.; Kim, T.-S.; Kim, B. J. Tuning Mechanical and Optoelectrical Properties of Poly(3-Hexylthiophene) through Systematic Regioregularity Control. Macromolecules 2015, 48 (13), 4339-4346. https://doi.org/10.1021/acs.macromol.5b00524.

(105) Menyhard, A.; Suba, P.; Laszlo, Zs.; Fekete, H. M.; Mester, A. O.; Horvath, Zs.; Voros, Gy.; Varga, J.; Moczo, J. Direct Correlation between Modulus and the Crystalline Structure in Isotactic Polypropylene. Express Polym. Lett. 2015, $9 \quad$ (3), 308-320. https://doi.org/10.3144/expresspolymlett.2015.28.

(106) Domke, J.; Radmacher, M. Measuring the Elastic Properties of Thin Polymer Films with the Atomic Force Microscope. Langmuir 1998, 14 (12), 3320-3325. https://doi.org/10.1021/la9713006.

(107) Jensen, F. Introduction to Computational Chemistry, 2nd ed.; John Wiley \& Sons: Chichester, England; Hoboken, NJ, 2007.

(108) Szabo, A.; Ostlund, N. S. Modern Quantum Chemistry: Introduction to Advanced Electronic Structure Theory; Courier Corporation, 1996.

(109) Hohenberg, P.; Kohn, W. Inhomogeneous Electron Gas. Phys. Rev. 1964, 136 (3B), B864B871. https://doi.org/10.1103/PhysRev.136.B864. 
(110) Kohn, W.; Sham, L. J. Self-Consistent Equations Including Exchange and Correlation Effects. Phys. Rev. 1965, 140 (4A), A1133-A1138. https://doi.org/10.1103/PhysRev.140.A1133.

(111) Sun, Y. Y.; Lee, K.; Wang, L.; Kim, Y.-H.; Chen, W.; Chen, Z.; Zhang, S. B. Accuracy of Density Functional Theory Methods for Weakly Bonded Systems: The Case of Dihydrogen Binding on Metal Centers. Phys. Rev. B 2010, 82 (7), 073401. https://doi.org/10.1103/PhysRevB.82.073401.

(112) Stephens, P. J.; Devlin, F. J.; Chabalowski, C. F.; Frisch, M. J. Ab Initio Calculation of Vibrational Absorption and Circular Dichroism Spectra Using Density Functional Force Fields. J. Phys. Chem. 1994, 98 (45), 11623-11627. https://doi.org/10.1021/j100096a001.

(113) Slater, J. C. A Simplification of the Hartree-Fock Method. Phys. Rev. 1951, 81 (3), 385-390. https://doi.org/10.1103/PhysRev.81.385.

(114) Becke, A. D. Density-Functional Exchange-Energy Approximation with Correct Asymptotic Behavior. Phys. Rev. A 1988, 38 (6), 3098-3100. https://doi.org/10.1103/PhysRevA.38.3098.

(115) Lee, C.; Yang, W.; Parr, R. G. Development of the Colle-Salvetti Correlation-Energy Formula into a Functional of the Electron Density. Phys. Rev. B 1988, 37 (2), 785-789. https://doi.org/10.1103/PhysRevB.37.785.

(116) Vosko, S. H.; Wilk, L.; Nusair, M. Accurate Spin-Dependent Electron Liquid Correlation Energies for Local Spin Density Calculations: A Critical Analysis. Can. J. Phys. 1980, 58 (8), 12001211. https://doi.org/10.1139/p80-159.

(117) Chai, J.-D.; Head-Gordon, M. Long-Range Corrected Hybrid Density Functionals with Damped Atom-Atom Dispersion Corrections. Phys. Chem. Chem. Phys. 2008, 10 (44), 6615-6620. https://doi.org/10.1039/B810189B.

(118) Chai, J.-D.; Head-Gordon, M. Systematic Optimization of Long-Range Corrected Hybrid Density Functionals. J. Chem. Phys. 2008, 128 (8), 084106. https://doi.org/10.1063/1.2834918.

(119) Tomasi, J.; Mennucci, B.; Cammi, R. Quantum Mechanical Continuum Solvation Models. Chem. Rev. 2005, 105 (8), 2999-3094. https://doi.org/10.1021/cr9904009.

(120) Frenkel, D.; Smit, B. Understanding Molecular Simulation: From Algorithms to Applications; Elsevier, 2001.

(121) Verlet, L. Computer "Experiments" on Classical Fluids. I. Thermodynamical Properties of Lennard-Jones Molecules. Phys. Rev. 1967, 159 (1), 98-103. https://doi.org/10.1103/PhysRev.159.98.

(122) Hockney, R. W.; Goel, S. P.; Eastwood, J. W. Quiet High-Resolution Computer Models of a Plasma. J. Comput. Phys. 1974, 14 (2), 148-158. https://doi.org/10.1016/0021-9991(74)90010-2.

(123) Swope, W. C.; Andersen, H. C.; Berens, P. H.; Wilson, K. R. A Computer Simulation Method for the Calculation of Equilibrium Constants for the Formation of Physical Clusters of Molecules: Application to Small Water Clusters. J. Chem. Phys. 1982, 76 (1), 637-649. https://doi.org/10.1063/1.442716.

(124) Wang, J.; Wolf, R. M.; Caldwell, J. W.; Kollman, P. A.; Case, D. A. Development and Testing of a General Amber Force Field. J. Comput. Chem. 2004, 25 (9), 1157-1174. https://doi.org/10.1002/jcc.20035.

(125) Saito, M. Molecular Dynamics Simulations of Proteins in Solution: Artifacts Caused by the Cutoff Approximation. J. Chem. Phys. 1994, 101 (5), 4055-4061. https://doi.org/10.1063/1.468411.

(126) Hockney, R. W.; Eastwood, J. W. Computer Simulation Using Particles; CRC Press, 1988. 
(127) Pollock, E. L.; Glosli, J. Comments on P3M, FMM, and the Ewald Method for Large Periodic Coulombic Systems. 18.

(128) Toukmaji, A. Y.; Board, J. A. Ewald Summation Techniques in Perspective: A Survey. 20.

(129) Shinoda, W.; Shiga, M.; Mikami, M. Rapid Estimation of Elastic Constants by Molecular Dynamics Simulation under Constant Stress. Phys. Rev. B 2004, 69 (13), 134103. https://doi.org/10.1103/PhysRevB.69.134103.

(130) Martyna, G. J.; Tobias, D. J.; Klein, M. L. Constant Pressure Molecular Dynamics Algorithms. J. Chem. Phys. 1994, 101 (5), 4177-4189. https://doi.org/10.1063/1.467468.

(131) Parrinello, M.; Rahman, A. Polymorphic Transitions in Single Crystals: A New Molecular Dynamics Method. J. Appl. Phys. 1981, 52 (12), 7182-7190. https://doi.org/10.1063/1.328693.

(132) Andersen, H. C. Molecular Dynamics Simulations at Constant Pressure and/or Temperature. $J$. Chem. Phys. 1980, 72 (4), 2384-2393. https://doi.org/10.1063/1.439486.

(133) Schneider, T.; Stoll, E. Molecular-Dynamics Study of a Three-Dimensional One-Component Model for Distortive Phase Transitions. Phys. Rev. B 1978, 17 (3), 1302-1322. https://doi.org/10.1103/PhysRevB.17.1302.

(134) Dünweg, B.; Paul, W. Brownian Dynamics Simulations without Gaussian Random Numbers. Int. J. Mod. Phys. C 1991, 02 (03), 817-827. https://doi.org/10.1142/S0129183191001037.

(135) Kim, N.; Lienemann, S.; Petsagkourakis, I.; Alemu Mengistie, D.; Kee, S.; Ederth, T.; Gueskine, V.; Leclère, P.; Lazzaroni, R.; Crispin, X.; Tybrandt, K. Elastic Conducting Polymer Composites in Thermoelectric Modules. Nat. Commun. 2020, 11 (1), 1424. https://doi.org/10.1038/s41467-02015135-w.

(136) Teo, M. Y.; Kim, N.; Kee, S.; Kim, B. S.; Kim, G.; Hong, S.; Jung, S.; Lee, K. Highly Stretchable and Highly Conductive PEDOT:PSS/Ionic Liquid Composite Transparent Electrodes for Solution-Processed Stretchable Electronics. ACS Appl. Mater. Interfaces 2017, 9 (1), 819-826. https://doi.org/10.1021/acsami.6b11988. 


\section{Publications}





\section{Papers}

The papers associated with this thesis have been removed for copyright reasons. For more details about these see:

https://doi.org/10.3384/9789179291440 


\section{FACULTY OF SCIENCE AND ENGINEERING}

Linköping Studies in Science and Technology, Dissertation No. 2194, 2021 Department of Science and Technology (ITN)

Linköping University

SE-581 83 Linköping, Sweden

www.liu.se 